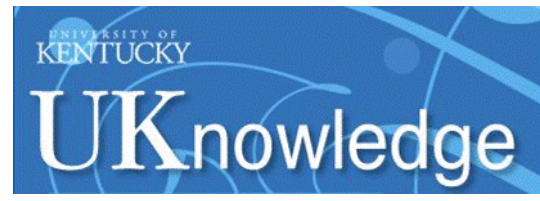

University of Kentucky

UKnowledge

\title{
NUMERICAL ANALYSIS OF METAL TRANSFER IN GAS METAL ARC WELDING
}

\author{
Ge Wang \\ University of Kentucky, gwang1@engr.uky.edu
}

Right click to open a feedback form in a new tab to let us know how this document benefits you.

\section{Recommended Citation}

Wang, Ge, "NUMERICAL ANALYSIS OF METAL TRANSFER IN GAS METAL ARC WELDING" (2007).

University of Kentucky Doctoral Dissertations. 538.

https://uknowledge.uky.edu/gradschool_diss/538

This Dissertation is brought to you for free and open access by the Graduate School at UKnowledge. It has been accepted for inclusion in University of Kentucky Doctoral Dissertations by an authorized administrator of UKnowledge. For more information, please contact UKnowledge@lsv.uky.edu. 


\section{ABSTRACT OF DISSERTATION}

Ge Wang

The Graduate School

University of Kentucky

2007 


\title{
NUMERICAL ANALYSIS OF \\ METAL TRANSFER IN GAS METAL ARC WELDING
}

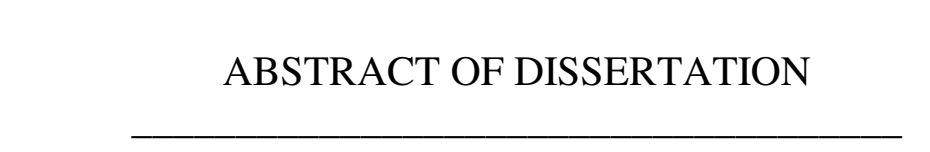

A dissertation submitted in partial fulfillment of the requirements for the degree of Doctor of Philosophy in the

College of Engineering at the University of Kentucky

\author{
By \\ Ge Wang \\ Lexington, Kentucky
}

Co-Directors: Dr. George P. Huang, Professor of Mechanical Engineering and

Dr. Yu-Ming Zhang, Professor of Electrical and Computer Engineering

Lexington, Kentucky

2007

Copyright (C) Ge Wang 2007 


\section{ABSTRACT OF DISSERTATION}

\section{NUMERICAL ANALYSIS OF METAL TRANSFER IN GAS METAL ARC WELDING}

In gas metal arc welding (GMAW), metal transfer plays a crucial role in determining the quality of the resultant weld. In the present dissertation, a numerical model with advanced computational fluid dynamics (CFD) techniques has been developed first in order to provide better numerical results. It includes a two-step projection method for solving the incompressible fluid flow; a volume of fluid (VOF) method for capturing free surface; and a continuum surface force (CSF) model for calculating surface tension. The Gauss-type current density distribution is assumed as the boundary condition for the calculation of the electromagnetic force. The droplet profiles, electric potential and velocity distributions within the droplet are calculated and presented for different metal transfer modes. The analysis is conducted to find the most dominant effects influencing the metal transfer behavior. Comparisons between calculated results and experimental results for metal transfer under constant current are presented and show good agreement.

Then, our numerical model is used to study a proposed modified pulsed current gas metal arc welding. This novel modified pulsed current GMAW is introduced to improve the robustness of the welding process in achieving a specific type of desirable and repeatable metal transfer mode, i.e., one drop per pulse (ODPP) mode. This new technology uses a peak current lower than the transition current to prevent accidental 
detachment and takes advantage of the downward momentum of the droplet oscillation to enhance the detachment. The calculations are conducted to demonstrate the effectiveness of the proposed method in achieving the desired metal transfer process in comparison with conventional pulsed current GMAW. Also, the critical conditions for effective utilization of this proposed method are identified by the numerical simulation. The welding operational parameters and their ranges are also calculated and the calculated results further demonstrate the robustness of this new GMAW technique in achieving high quality welding.

KEYWORDS: Gas Metal Arc Welding, Metal Transfer, Pulsed Current GMAW, ODPP Metal Transfer, Numerical Analysis

Ge Wang

07/10/2007 
Ge Wang

George P. Huang

Co-Director of Dissertation

Yu-Ming Zhang

Co-Director of Dissertation

L. Scott Stephens

Director of Graduate Studies

$7 / 10 / 2007$

Date 


\section{RULES FOR THE USE OF DISSERTATIONS}

Unpublished dissertations submitted for the Doctor's degree and deposited in the University of Kentucky Library are as a rule open for inspection, but are to be used only with due regard to the rights of the authors. Bibliographical references may be noted, but quotations or summaries of parts may be published only with the permission of the author, and with the usual scholarly acknowledgments.

Extensive copying or publication of the dissertation in whole or in part also requires the consent of the Dean of the Graduate School of the University of Kentucky.

A library that borrows this dissertation for use by its patrons is expected to secure the signature of each user.

$\underline{\text { Name }}$

Date 


\section{DISSERTATION}

Ge Wang

The Graduate School

University of Kentucky

2007 


\title{
NUMERICAL ANALYSIS OF \\ METAL TRANSFER IN GAS METAL ARC WELDING
}

DISSERTATION

A dissertation submitted in partial fulfillment of the requirements for the degree of Doctor of Philosophy in the College of Engineering at the University of Kentucky

\author{
By \\ Ge Wang \\ Lexington, Kentucky
}

Co-Directors: Dr. George P. Huang, Professor of Mechanical Engineering and Dr. Yu-Ming Zhang, Professor of Electrical and Computer Engineering

Lexington, Kentucky

2007

Copyright $\odot$ Ge Wang 2007 


\section{ACKNOWLEDGMENTS}

First of all, I would like to express my sincere thanks to my family, teachers, friends and those who inspired and helped me to acquire a doctoral degree during the entire process of this study.

Special thanks should go to Dr. George P. Huang, my major professor and chairman of the advisory committee, for his continuous support and encouragement, insightful advice, and instructive comments on this dissertation. Carrying out this research would not have been possible without the help from my co-director Dr. YuMing Zhang. I thank him for generously sharing his knowledge in welding experiments and providing continuous guidance throughout this research. At the same time, I would also like to thank the other members of my advisory committee: Dr. Raymond P. LeBeau and Dr. Vincent Capece for their review and giving valuable suggestions toward this dissertation.

I also would like to acknowledge the financial supports from Department of Mechanical Engineering at University of Kentucky and Center for Robotics and Manufacturing Systems at University of Kentucky.

Last, but not the least, I want to thank my wonderful husband, Sean, and my lovely daughter, Wendy. I wish I could express in words how much I appreciate their support, understanding, and patience. 


\section{TABLE OF CONTENTS}

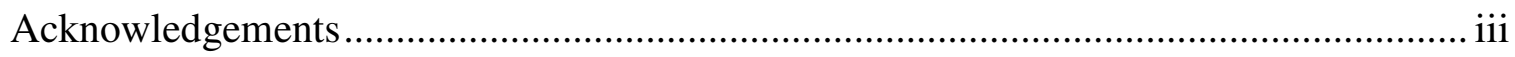

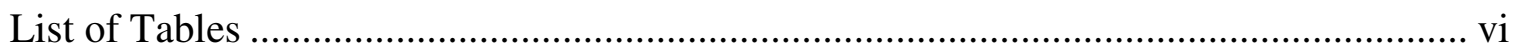

List of Figures ................................................................................................... vii

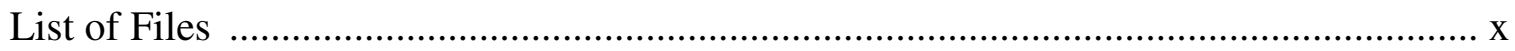

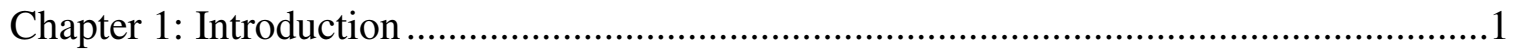

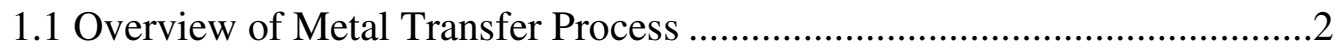

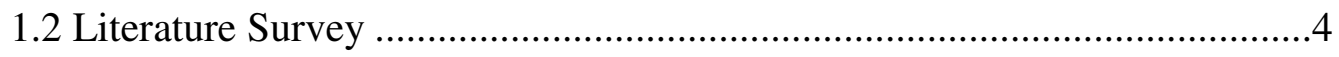

1.3 Motivation and Objectives .......................................................................13

1.4 Dissertation Organization ......................................................................15

Chapter 2: Physical Model of Metal Transfer..................................................................

2.1 Physical Process of Metal Transfer............................................................17

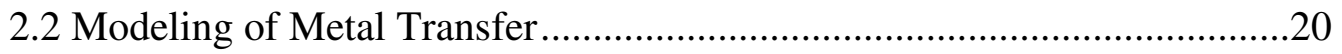

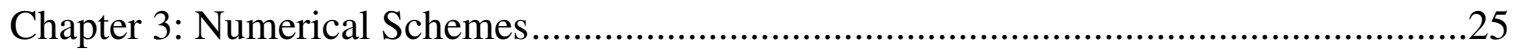

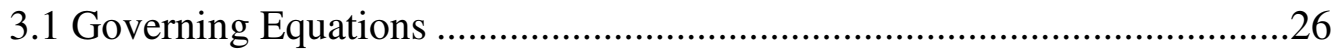

3.2 Numerical Modeling and Solution for Governing Equations .....................27

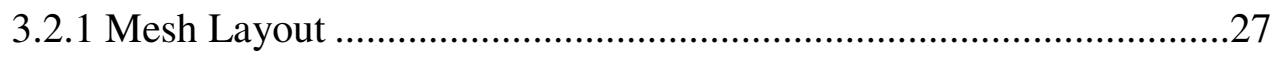

3.2.2 Discretization of Governing Equations and Algorithm Solution.......29

a. The Two-step Projection Method......................................................30

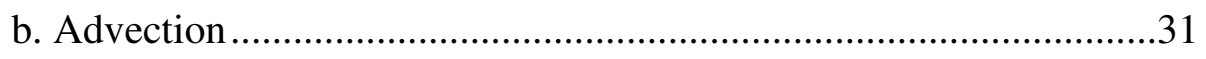

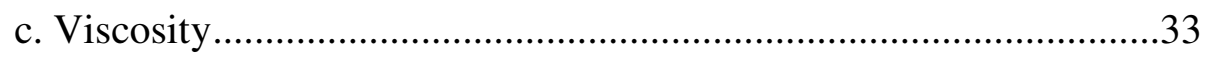

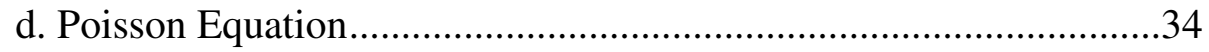

3.3 Tracking the Free Surface ...........................................................................38

3.4 Modeling of Surface Tension..................................................................4

3.5 Calculation of Electromagnetic Force ……………….............................46 
Chapter 4: Metal Transfer in Constant Current GWAW …...........................................54

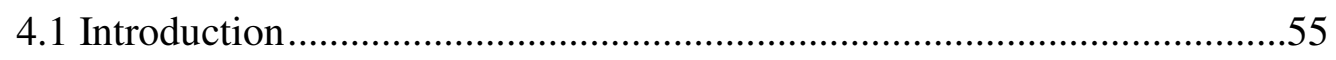

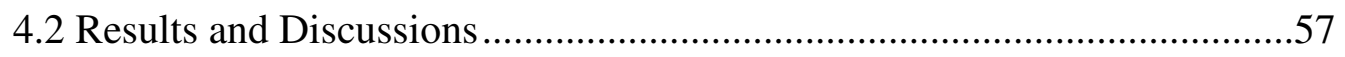

4.2.1 Effects of Surface Current Density Distribution ...........................58

4.2.2 Dominant Effects for Different Metal Transfer Mode.......................60

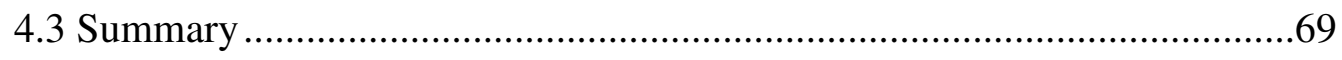

Chapter 5: Metal transfer in Pulsating Current GMAW .............................................. 71

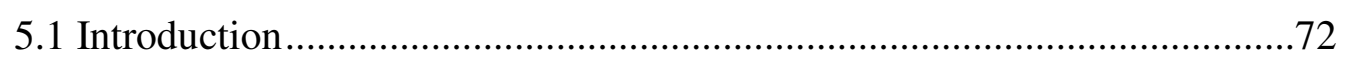

5.2 Proposed approach in modified pulsed current GMAW ..........................76

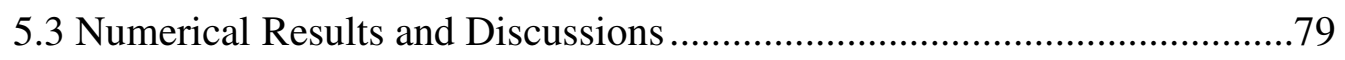

5.3.1 Traditional Single Pulsed Current GMAW ....................................81

5.3.2 Modified Pulsed Current GMAW ................................................89

5.3.3 Parameter Diagnoses for Phase Match ..........................................992

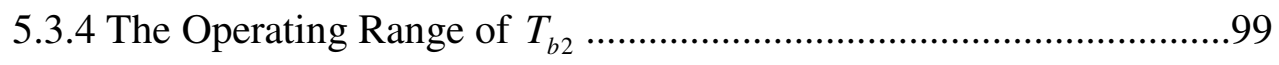

5.3.5 The Influence of Pulsing Cycle Frequency and Peak Current.........106

5.3.6 Comparison with Experiment ...................................................122

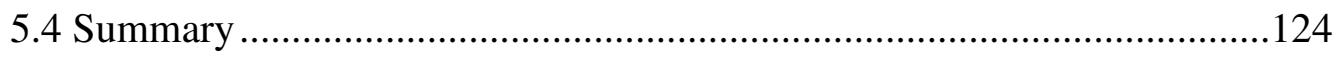

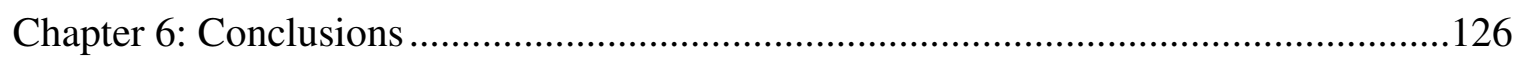

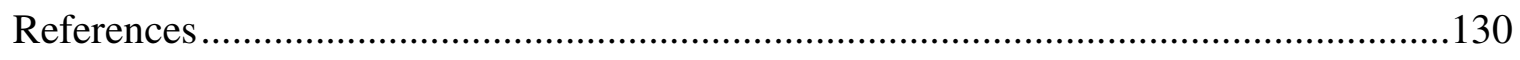

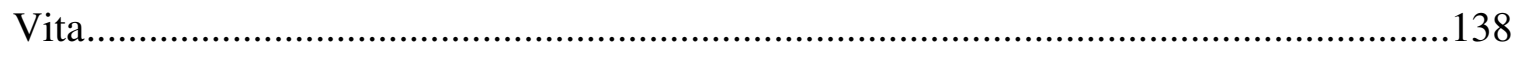




\section{LIST OF TABLES}

Table 1, Material Properties of the Electrode ................................................................19 


\section{LIST OF FIGURES}

Figure 2.1, The sketch of metal transfer process in GMAW …...................................17

Figure 2.2, Schematic sketch of metal transfer process in GMAW with initial and boundary conditions: (a) A Schematic of metal transfer process; (b) Initial and external

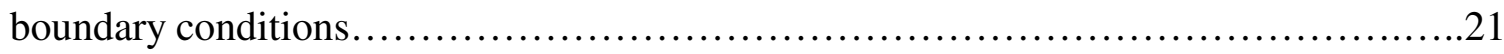

Figure 3.1, Flow diagram of numerical solution......................................................27

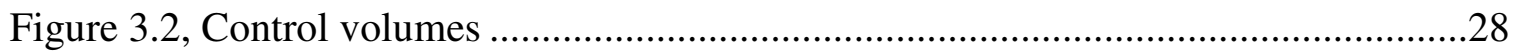

Figure 3.3, Checker-board distribution for pressure .................................................29

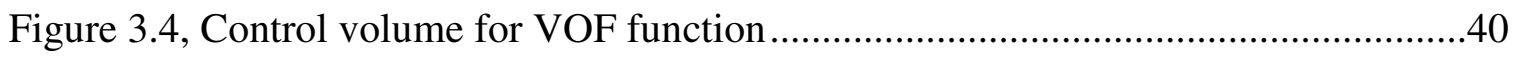

Figure 3.5, Examples of free surface shapes and reconstructions in the advection of $F$

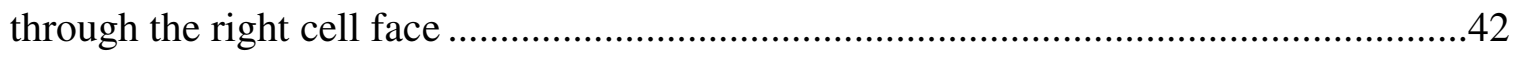

Figure 3.6, The transition zone with thickness $h$ at the interface ................................44

Figure 3.7, Layout of electromagnetic variables in a computational cell...............48

Figure 3.8, Examples of free surface cells using for boundary condition application......50

Figure 4.1, Comparison of predicted average droplet sizes under different current density distribution with experimental results

Figure 4.2, metal transfer process at the current of 160A: (a) Drop profiles (b) Electric potential and velocity distributions within the droplet

Figure 4.3, metal transfer process at the current of 300A: (a) Drop profiles (b) Electric potential and velocity distributions within the droplet

Figure 4.4, Metal Transfer Process at the Current of 250A: (a) Drop profiles (b) Electric

potential and velocity distributions within the droplet 68

Figure 5.1, Current waveform for conventional pulsed GMAW ....................................73

Figure 5.2, Current waveform used for modified pulsed GMAW ................................77

Figure 5.3, The relationship between the detached drop sizes and welding currents ........80

Figure 5.4 Metal transfer with ODPP under conventional pulsed current GMAW:

(a) Droplet profiles (b) Vertical coordinate of droplet tip

Figure 5.5, Comparison of calculated and experimental operating ranges:
(a) $I_{p}=400 \mathrm{~A}, I_{b}=180 \mathrm{~A}, I_{a v g}=202 \mathrm{~A}$
(b) $I_{p}=400 \mathrm{~A}, I_{b}=180 \mathrm{~A}, I_{a v g}=191 \mathrm{~A}$
(c) $I_{p}=500 \mathrm{~A}, I_{b}=180 \mathrm{~A}, I_{a v g}=196 \mathrm{~A}$
(d) $I_{p}=500 \mathrm{~A}, I_{b}=180 \mathrm{~A}, I_{a v g}=212 \mathrm{~A}$ 
Figure 5.6, Metal transfer with ODMP under conventional pulsed current GMAW .......88

Figure 5.7, Metal transfer with MDPP under conventional pulsed current GMAW

Figure 5.8, Metal transfer with ODPP under modified pulsed current GMAW:

(a) Current waveform (b) Droplet profiles (c) Vertical coordinate of droplet tip .92

Figure 5.9, Metal transfer processes with unsatisfied phase match condition: (a) Drop profiles under a shorter duration $T_{b 2}$ of $3 \mathrm{~ms}$ (b) Vertical coordinate of droplet tip

Figure 5.10 Metal transfer processes with unsatisfied phase match condition: (a) Drop profiles under a longer duration $\mathrm{T}_{\mathrm{b} 2}$ of $6 \mathrm{~ms}(\mathrm{~b})$ Vertical coordinate of droplet tip............96 Figure 5.11, The droplet response to exciting pulse: (a) Current waveform (b) Vertical coordinate of droplet tip

Figure 5.12 Development for vertical coordinate of droplet tip in modified pulsed current GMAW: (a) ODPP under $\mathrm{T}_{\mathrm{b} 2}$ of $3.5 \mathrm{~ms}$ (b) ODPP under $\mathrm{T}_{\mathrm{b} 2}$ of $4.5 \mathrm{~ms}$ (c) ODPP under $\mathrm{T}_{\mathrm{b} 2}$ of $5 \mathrm{~ms} \quad$ (d) ODPP under $\mathrm{T}_{\mathrm{b} 2}$ of $5.5 \mathrm{~ms}$ .98

Figure 5.13, Development for vertical coordinate of droplet tip in modified pulsed current GMAW $\left(T_{p l}=4 \mathrm{~ms}, I_{a v g}=100 \mathrm{~A}, f=30 \mathrm{~Hz}, I_{p}=220 \mathrm{~A}, I_{b}=40 \mathrm{~A}\right)$ : (a) ODMP under $\mathrm{T}_{\mathrm{b} 2}$ of $2.8 \mathrm{~ms}$

(b) ODPP under $\mathrm{T}_{\mathrm{b} 2}$ of $3 \mathrm{~ms}$ (c) ODPP under $\mathrm{T}_{\mathrm{b} 2}$ of $4 \mathrm{~ms}$ (d) ODPP under $\mathrm{T}_{\mathrm{b} 2}$ of $4.5 \mathrm{~ms}$

(e) ODPP under $\mathrm{T}_{\mathrm{b} 2}$ of $5 \mathrm{~ms}$ (f) ODMP under $\mathrm{T}_{\mathrm{b} 2}$ of $5.5 \mathrm{~ms}$ .101

Figure 5.14, Development for vertical coordinate of droplet tip in modified pulsed current GMAW ( $\left.T_{p 1}=3 \mathrm{~ms}, I_{a v g}=100 \mathrm{~A}, f=30 \mathrm{~Hz}, I_{p}=220 \mathrm{~A}, I_{b}=40 \mathrm{~A}\right)$ : (a) ODMP under $\mathrm{T}_{\mathrm{b} 2}$ of $2.5 \mathrm{~ms}$ (b) ODPP under $\mathrm{T}_{\mathrm{b} 2}$ of $2.8 \mathrm{~ms}$; (c) ODPP under $\mathrm{T}_{\mathrm{b} 2}$ of $3 \mathrm{~ms}$; (d) ODPP under $\mathrm{T}_{\mathrm{b} 2}$ of $4 \mathrm{~ms}$

$\begin{array}{ll}\text { (e) ODPP under } \mathrm{T}_{\mathrm{b} 2} \text { of } 5 \mathrm{~ms} & \text { (f) ODMP under } \mathrm{T}_{\mathrm{b} 2} \text { of } 5.5 \mathrm{~ms}\end{array}$ .103 Figure 5.15, Development for vertical coordinate of droplet tip in modified pulsed current GMAW ( $\left.T_{p 1}=6 \mathrm{~ms}, I_{a v g}=100 \mathrm{~A}, f=30 \mathrm{~Hz}, I_{p}=220 \mathrm{~A}, I_{b}=40 \mathrm{~A}\right)$ : (a) ODMP under $\mathrm{T}_{\mathrm{b} 2}$ of $4 \mathrm{~ms}$; (b) ODPP under $\mathrm{T}_{\mathrm{b} 2}$ of $4.5 \mathrm{~ms}$; (c) ODPP under $\mathrm{T}_{\mathrm{b} 2}$ of $5 \mathrm{~ms}$; (d) ODMP under $\mathrm{T}_{\mathrm{b} 2}$ of $5.5 \mathrm{~ms}$ .105

Figure 5.16, Development for vertical coordinate of droplet tip in modified pulsed current GMAW $\left(T_{p l}=7 \mathrm{~ms}, I_{a v g}=100 \mathrm{~A}, f=30 \mathrm{~Hz}, I_{p}=220 \mathrm{~A}, I_{b}=40 \mathrm{~A}\right)$ : (a) ODMP under $\mathrm{T}_{\mathrm{b} 2}$ of $4 \mathrm{~ms}$ (b) ODMP under $\mathrm{T}_{\mathrm{b} 2}$ of $5 \mathrm{~ms}$ .106 Figure 5.17, Metal transfer with ODPP under modified pulsed current GMAW:

(a) Current Signal (b) Droplet profiles (c) Vertical coordinate of droplet tip .108 
Figure 5.18, Development for vertical coordinate of droplet tip in modified pulsed current GMAW: (a) ODMP under $\mathrm{T}_{\mathrm{b} 2}$ of 2.5ms; (b) ODPP under $\mathrm{T}_{\mathrm{b} 2}$ of 3ms; (c) ODPP under $\mathrm{T}_{\mathrm{b} 2}$ of $3.5 \mathrm{~ms}$; (d) ODMP under $\mathrm{T}_{\mathrm{b} 2}$ of $4 \mathrm{~ms}$ .109

Figure 5.19, Metal transfer with ODPP under modified pulsed current GMAW:

(a) Current waveform (b) Droplet profiles (c) Vertical coordinate of droplet tip 111

Figure 5.20 Development for vertical coordinate of droplet tip in modified pulsed current GMAW: (a) ODMP under $\mathrm{T}_{\mathrm{b} 2}$ of $2.5 \mathrm{~ms}$ (b) ODPP under $\mathrm{T}_{\mathrm{b} 2}$ of $3 \mathrm{~ms}$ (c) ODPP under $\mathrm{T}_{\mathrm{b} 2}$ of $4 \mathrm{~ms}$ (d) ODMP under $\mathrm{T}_{\mathrm{b} 2}$ of $4.5 \mathrm{~ms}$

Figure 5.21, Development for vertical coordinate of droplet tip in modified pulsed current GMAW ( $\left.I_{p 1}=220, I_{p 2}=250\right)$ : (a) ODPP under $\mathrm{T}_{\mathrm{b} 2}$ of $3 \mathrm{~ms} \quad$ (b) ODPP under $\mathrm{T}_{\mathrm{b} 2}$ of $4 \mathrm{~ms}$ (c) ODPP under $\mathrm{T}_{\mathrm{b} 2}$ of $5 \mathrm{~ms} \quad$ (d) ODPP under $\mathrm{T}_{\mathrm{b} 2}$ of $6 \mathrm{~ms}$. .114 Figure 5.22, Metal transfer with MDPP under conventional pulsed current GMAW:

(a) Vertical coordinate of droplet tip (b) Droplet profiles 116

Figure 5.23, Development for vertical coordinate of droplet tip in modified pulsed current GMAW ( $I_{p 1}=220, I_{p 2}=300, T_{p 1}=5 \mathrm{~ms}$ ): (a) ODPP under $\mathrm{T}_{\mathrm{b} 2}$ of $2 \mathrm{~ms}$; (b) ODPP under $\mathrm{T}_{\mathrm{b} 2}$ of 4ms; (c) ODPP under $\mathrm{T}_{\mathrm{b} 2}$ of 6ms; (d) ODPP under $\mathrm{T}_{\mathrm{b} 2}$ of 7ms; (e) ODPP under $\mathrm{T}_{\mathrm{b} 2}$ of $10 \mathrm{~ms}$; (f) ODPP under $\mathrm{T}_{\mathrm{b} 2}$ of $20 \mathrm{~ms}$ 118 Figure 5.24, Drop velocities toward welding pool under different detaching pulse current

Figure 5.25, Comparison between the calculated results and experimental data: (a) Current waveforms (b) Vertical coordinate of droplet tip from experiment (c) Vertical coordinate of droplet tip from calculation .123 


\section{LIST OF FILES}

1. WangGe_Dissertation.pdf: 4 MB (File Size) 


\section{Chapter 1}

\section{Introduction}

In gas metal arc welding (GMAW), many effects influence the welding quality. Among these, metal transfer plays a crucial role in determining the quality of the resultant weld. Metal transfer describes the process of the molten metal movement from the electrode tip to the workpiece across the arc in gas metal arc welding. In order to achieve high quality welding, the manner in which the liquid metal transfers from the electrode to the weld pool has been the subject of much research. A better understanding of the metal transfer process not only helps to optimize and refine the welding process, but also provides opportunities to develop new techniques for high quality welding. In the past, empirical approaches have been applied with much success, but this approach is highly time consuming. A theoretical analysis has the advantage of providing insight into the underlying physics of the process. In this thesis, a method has been proposed to pulsate the current in GMAW to achieve a specific type of desirable and repeatable metal transfer mode. Extensive efforts have been made to explore the mechanism of the metal transfer process and understand the underlying physics of the process numerically. The numerical analysis applied in this thesis not only provides significant insights into the metal transfer process in general, but also provides an effective means to diagnose the optimum operation parameters for the proposed new technique. 


\subsection{Overview of Metal Transfer Process}

Gas metal arc welding is an important and widely used metal joining method in many industrial and manufacturing operations. During gas metal arc welding, the electrode is melted and liquid droplets are formed at the tip of the electrode. The melted metal grows at the end of electrode and is detached from the electrode. This process is referred to as metal transfer process. Previous studies [1-5] showed that the behavior of metal transfer affects the welding quality in many ways.

In gas metal arc welding, metal transfer can take place in three major distinct modes: globular, spray, and short-circuiting [6-8]. Spray transfer can be further classified as drop spray or streaming spray, depending on the diameter of the detached droplet in relation to that of the electrode: approximately the same in drop spray or much smaller in streaming spray. At low current, globular transfer occurs if the arc length is sufficient. The droplets grow at the tip of the electrode with a classic pendant drop shape, due to the competition between gravity and surface tension in the presence of relatively small electromagnetic forces. Large droplets with diameters much greater than the diameter of the electrode are detached primarily by gravity. When the welding current increases, the electromagnetic force becomes the dominant droplet force so that small droplets with diameter equal to or less than the diameter of the electrode can be detached. This is referred to as the spray transfer mode. It is found that there is an abrupt transition in the current, which divides the globular and spray transfer modes. This current or current range is referred to as the transition current. High irregularity in the droplet detachment 
frequency and the droplet size has been observed in the middle of the transition current range [8-10]. Short-circuiting transfer [11] is a special transfer mode while the molten droplet makes direct contact with the weld pool. It is characterized by intermittent arc extinguishment and re-ignition.

Globular and short-circuiting metal transfer typically causes significant spatters and poor welding quality [12]. Its application in production is limited. The spray transfer mode has advantages over the other metal transfer modes with its regular detachment accompanied by uniform droplet size, directional droplet transfer, and low spatters [13]. However, spray transfer is only achieved at high current for constant current GMAW, which results in a thermal load too high to apply to thin sectioned or heat-sensitive materials. Thereby, its application is restricted. In an effort to overcome this difficulty, pulsed current GMAW was introduced in 1962 [14]. By using a pulsed current, a controlled spray transfer mode with one droplet detached per pulse could be achieved at low average current, which typically results in globular transfer for constant current GMAW. Such a metal transfer mode is referred to as one-drop per-pulse (ODPP).

The change of the character of metal transfer affects the welding quality in many ways. For this reason, it has been and is still being investigated very intensively both experimentally and theoretically by researchers around the world. 


\subsection{Literature Survey}

The experimental techniques [15-24], which have been widely used in previous studies of the metal transfer process, include optical methods, sensor measurements, and acoustic detections. In the early 1980's, an optical technique (100-1000 frames per second) was developed at the M.I.T. welding laboratory for viewing metal transfer process with a relatively small aggregate of optical equipment [15]. This technique may be used to obtain the temporal evolution of the profile of droplet detachment from a gasshielded welding electrode. Lawrence A. Jones and his associates collected an extensive set of clear images of drop detachment in 1995 by using a high-speed video recorder with speeds from 2000 to 6000 frames per second [16]. The experiments recorded include a wide range of constant current and pulsed current welds using steel and a smaller set of aluminum welds. The clear high-speed images and associated data provided by this technique are a major contribution to the study of metal transfer process in GMAW. Optical methods mainly involve high-speed video systems and laser shadowing techniques [15-18]. This makes the cost very high. The arc sensor is also widely used in GMAW to study the process [19-22]. By recording and analyzing fluctuations of the welding voltage and/or current, it is possible to predict the metal transfer mode. But it cannot provide any detail or significant insights about the metal transfer process. Its application is better suited to welding process control. As another approach, Manz studied the relationship between the sound of a welding arc and the metal transfer mode by acoustic measurement [23]. Since acoustic signals can easily be disturbed by the background noise, the reliability of this method is questionable. 
Theoretical description of metal transfer in GMAW can provide a better understanding of the mechanism of this process and the means to determine the optimal operation parameters. However, theoretical description of droplet formation and detachment in GMAW are complicated by the following effects: the dynamic nature of droplet growth, thermal phenomena in the wire, and heat transfer from the arc. Because of the complexities associated with these effects, models in the literature for prediction of metal transfer in GMAW are typically based on simplified descriptions of the effects influencing the process of droplet formation. Numerous models have been developed to study the metal transfer process in GMAW.

The two best-known models developed from early studies of metal transfer analysis are the static force balance theory (SFBT) [25-27] and the pinch instability theory (PIT) [28-30].

The static force balance theory (SFBT) was first proposed by Greene [25] and further developed by Amson [26,27] and Waszink et al [1]. It predicts the detaching drop size by simply comparing the balance between attaching and detaching forces. The main attaching force is the surface tension force. Detaching forces include gravitational force, electromagnetic force and plasma drag force. The drop detaches when the detaching force becomes greater than the attaching force. Since this model is based on static force analysis, the dynamic character of metal transfer cannot be considered by SFBT. Also, this model does not take consideration of droplet shape and neglects interaction between 
droplet shape and influential forces. Predicted results based on SFBT show severe deviation from experimental data at higher current while reasonable agreement is achieved at low current.

As an extension of the static force balance model, Choi et al [31] proposed a dynamic force balance model (DFBM) for metal transfer analysis. The dynamic force balance model predicts metal transfer in arc welding by introducing the inertial force in addition to the conventional forces used in the SFBT. The dynamics of a pendent drop are modeled as a second-order mass, spring, and damper system. Although the DFBM shows better agreement with the measured drop size than the SFBT, both models are unable to accurately predict the detached drop size in the high current range.

The pinch instability theory (PIT) was first applied to GMAW by Lancaster [6]. Allum $[28,29]$ further used it to predict the detached drop size in metal transfer. Rhee and Kannatey [30] extended the PIT to include effects of arc pressure. The PIT predicts the droplet size based on consideration of the instability of the current-carrying liquid cylindrical column. The PIT considers perturbation due to the radial magnetic pinch force acting on an infinite cylindrical column of liquid metal. According to Rayleigh instability theory, the disturbance in the fluid cylinder can grow exponentially and break it into droplets. The size of the droplets depends on the wavelength of the fastest growing disturbance. This model oversimplifies the droplet shape. Predictions made according to PIT provide the correct order of magnitude of the detached droplet radius at higher current, but have major discrepancies with experimental data at low current. 
Both SFBT and PIT fail to describe metal transfer properly over a wide current range due to the oversimplification of those two models. Neither can predict the transition from globular transfer mode to spray transfer mode successfully. Other models have been proposed to predict metal transfer more accurately.

In 1994, Nemchinsky [32] developed a steady-state model to describe metal transfer by calculating the equilibrium shape of a pendant droplet. An equation to describe the droplet shape is proposed and solved. It calculates the maximum volume of droplet that can still be attached to the electrode, and then computes the radius of the detached droplet. This model is the first to include effects coming from the coupling between surface tension, electromagnetic force, and the droplet shape. It allows calculation of the detaching droplet size more accurately over a wider current range compared to the SFBT and PIT models.

In 1996, Joo et al [33] presented a numerical model based on the energy minimization method to calculate the molten drop geometry. The gravitational, surface tension and electromagnetic forces are considered in order to formulate the energy of the pendant droplet system, and therefore influence the geometry of the static pendant drop. The dominant effects are identified for different metal transfer modes. The drop profile is mainly affected by the surface tension and electromagnetic force in the spray transfer mode. Effects of the gravitational force increase in the globular transfer mode. 
Predictions agree favorably with experimental data in the globular mode and the initial stage of the spray mode.

However, the above two models are basically static approaches. They are still unable to predict the dynamic behavior of the droplet growth and detachment during metal transfer. The calculations tend to diverge as soon as the instability occurs. The dynamic description of the droplet development and detachment process is critical to understanding the details of metal transfer in GMAW.

In 1995, Simpson and Zhu [34] developed a dynamic one-dimensional model to predict droplet formation and detachment. It includes dynamic development of droplet shape and size under the action of gravity, electromagnetic forces and surface tension. This model provided the first predictions of droplet shape as a function of time. However, it is not suitable for making adequate predictions of the transition current between globular and spray transfer mode, nor does it describe the details of the metal transfer process.

In 1998, Jones and Eagar [35,36] presented a dynamic model of drop detachment for low and moderate welding currents in gas metal arc welding. The dynamic model they developed explicitly considers the geometry of drops as they detach from an electrode, thereby providing a detailed view of how the forces acting on the drops evolve. This dynamic model is a lumped parameter system in nature. Forces are applied to the center of mass, rather than being applied in a continuum way to the distributed mass of 
the droplet. Comparisons with experiment indicate that the calculated axial magnetic forces are substantially too high when using constant current.

Recently, the rapid development of high-speed computers has made significant contribution to the progress of Computational Fluid Dynamics (CFD) techniques. Several transient, two-dimensional models have been developed to predict metal transfer process based on advanced CFD techniques.

In 1996, Haidar and Lowke [38-40] developed a two-dimensional dynamic model for the prediction of droplet formation that included the arc. This was the first time that an advanced CFD technique such as the VOF method was employed to study metal transfer and it made a great impact on this field. Equations of continuity, momentum, energy and current were solved in two dimensions for the molten droplet and arc. This model predicted the transition current from the globular to the spray transfer mode in fair agreement with experimental data. However, this model failed to predict the presence of both small and large drops in the transition zone between the two modes. The droplet detachment was not addressed and the shape of drop was not very close to the images given by experiments. The accuracy of computational results is influenced by discontinuity assumptions on the free surface, such as a surface pressure boundary condition.

In 1998, a mathematical model to describe the globular transfer was developed by Fan and Kovacevic [41,42]. The droplet formation, detachment and transport 
phenomena are considered together with the impingement effect on the weld pool. The fluid flow and heat transfer in metal transfer are dynamically studied by using the VOF method in a two-dimensional domain. An approximation was used to obtain the current density distribution in the droplet by assuming uniform axial current density distribution over the horizontal cross section of the droplet. The size and the transfer frequency of the droplets of globular transfer are determined by the balance of gravity, surface tension, electromagnetic, and arc drag forces. The calculated results agree well with the experimental results recorded by a high-speed video camera. However, the calculation was carried out only for globular transfer.

Chio, Kim and Yoo [43] also conducted numerical simulations of metal transfer in 1998. They considered the effect of the welding arc under the assumptions of a uniform and linear current density on the droplet surface. The dynamic characteristics of the globular, spray and short-circuit metal transfer modes were simulated by adopting the VOF method. They noted that the current density on the drop surface has significant effects on the shape and size of droplet. They further did a dimensional analysis in order to determine the dominant factors that affect the metal transfer mode [44]. They found the ratio of the electromagnetic force and the surface tension force have the largest effects on the metal transfer characteristics over the whole range of welding conditions. The predicted results are in reasonably good agreement with the experimental data, although the transition current and characteristic of metal transfers occurring in this transition current range are not determined accurately. 
In 2001, Wang and his associates [45-47] successfully conducted numerical analysis for the droplet impingement on the weld pool surface and the fluid flow, heat, mass transfer in the weld pool for GMAW. The RIPPLE computer program, which models transient, two-dimensional, incompressible fluid flows with free surfaces by using advanced CFD techniques, was introduced into the study of GMAW. While their study focused on the interaction between the droplet and the weld pool, the droplet growth and detachment process was not included in their paper.

The necessary conditions to achieve the desirable one drop per pulse (ODPP) mode, which characterizes a stable, periodical, and controllable metal transfer process, were investigated in a number of works [48-57].

To this end, Ueguri et al [48] analyzed the metal transfer in pulsed GMAW by using static force balance theory in 1985. They pointed out the significance of the optimum current waveform on achieving the ODPP metal transfer mode. They also suggested the peak current should be set above a critical current to ensure that one droplet is detached per pulse. Amin [49] identified the critical current to ensure ODPP metal transfer, which was the transition current between the globular and spray transfer mode.

In the pulsed current GMAW, the current waveform is regarded as an important operation condition to achieve ODPP metal transfer. In order to obtain one drop per pulse, Quintino [50,51] suggested that the peak duration $T_{p}$ should be decreased when the peak current $I_{p}$ increases. Smati [52] predicted the theoretical pulsing frequency, and 
showed that one drop per pulse is realized when the term $I_{p}^{2} T_{p}$ remains constant. The work of Kim [54-56] and his associates pointed out that there is a range of operational parameters within which one droplet is transferred per current pulse. The operating range of the pulsing frequency $f$, which provides ODPP, was found to increase when the peak current $I_{p}$ or load duty cycle $T_{p} f$ increased. The study conducted by Nemchinsky [57] considers the electrode-melting rate under pulsed current GMAW. The results further confirmed that there is a range of pulsing frequency leading to ODPP metal transfer.

Previous models for the metal transfer process have been unable to make accurate predictions of the transition between the globular and spray transfer modes. In the present study [58,59], a new transient two-dimensional model is developed on the base of RIPPLE [60] to simulate the droplet formation, detachment and transport in gas metal arc welding. The transient shape of the droplet is calculated using the fractional volume of fluid (VOF) method [61], which is shown to be more flexible and efficient than other methods for treating complicated free-boundary configurations. Gravitational force, surface tension force, and electromagnetic force play fundamental roles in the process of droplet growth and detachment. The continuum surface force (CSF) model [62] adopted in this study eliminates the need for interface reconstruction, simplifies the calculation of surface tension, and enables accurate modeling of fluid flows driven by surface forces. As the welding current generates the electromagnetic force exerted on the pendant drop, the effects of the current are included with assumption of Gaussian current density distribution. The numerical results [58] show a very good agreement with the experimental data. The transition current range and the special behavior of metal transfer 
during this range have been calculated and show good agreement with experimental observations. The analysis of the calculation results provides significant insight into the physical mechanisms, which influence the metal transfer procedure.

\subsection{Motivation and Objectives}

Metal transfer with one drop per pulse (ODPP) mode, which characterizes a stable, periodical, and controllable process, can produce high quality welding. Hence, its application is the most desirable. In conventional pulsed GMAW, the peak current has to be set above the transition current along with selection of the appropriate parameters to get the desirable one-drop per pulse mode (ODPP). On the other hand, it has been shown that a peak current above the transition current will easily bring accidental detachment, i.e. multiple drops detached per pulse (MDPP), and overheat the droplet and welding pool.

Recently, a novel active control technology has been proposed by E Zhang and his associates [63-65]. A pulse cycle composed of two pulses, exciting pulse and detaching pulse, is used to detach a drop per pulse cycle during gas metal arc welding. A peak current below the transition current is used to detach the droplet, prevent accidental detachment, and realize the optimal ODPP metal transfer mode. The drop is detached by the combination of the downward momentum of the drop oscillation and the increased electromagnetic force, which is induced by an exciting pulse and a detaching pulse, respectively. The phase match between the downward movement and the increased pulse 
current plays an important role to achieve ODPP metal transfer by utilizing this modified pulsed current arc welding.

The modified pulsed current arc welding has a major advantage over the conventional pulsed current GMAW in being capable of lowering the peak current under transition current to obtain ODPP metal transfer. On the other hand, this method introduces large amounts of additional welding parameters due to the use of double pulse waveforms. These extra variables cause difficulty in selecting optimum combinations of parameters for a wide range of welding conditions to realize ODPP metal transfer. A trial-and-error method has been used to determine these parameters experimentally. However, this empirical approach is very time consuming and unpractical. A theoretical description of metal transfer in GMAW not only provides a better understanding of the technology's mechanism, but also an efficient way to determine the optimum operation parameters.

The rapid development of Computational Fluid Dynamics (CFD) techniques has made great contribution to the progress of the theoretical study for GMAW. Hence, advanced CFD techniques have been adopted as an effective means in the present numerical study of metal transfer in GMAW. An effort has been made to get calculated results in better agreement with available experimental data by physical modeling and use of advanced numerical schemes. The analysis of numerical results not only give significant insights into the metal transfer process in general, but also provide an 
efficacious means to diagnose the optimum operation parameters for the proposed new technique and make this novel active control technology feasible in industry.

\subsection{Dissertation Organization}

First of all, the background, literature survey, motivation and objectives of this dissertation study have been introduced in Chapter 1. The physical model of metal transfer in GMAW will be presented in Chapter 2. The numerical schemes and algorithms to solve the governing equations are shown in Chapter 3. Chapter 4 includes the calculated results for preliminary test cases and the simulations for metal transfer in GMAW under constant currents. The simulations carried out for metal transfer under the pulsed current GMAW, which include the traditional pulsed current and modified pulsed current GMAW, and results are discussed in Chapter 5. Some standard numerical test cases are also included in this chapter for validation purposes. Finally, conclusions are provided in Chapter 6.

Copyright $\odot$ Ge Wang 2007 


\section{Chapter 2}

\section{Physical Model of Metal Transfer}

Metal transfer, the process of transferring welding wire material in the form of molten metal droplets to the workpiece in gas metal arc welding (GMAW), involves complex dynamic interactions between many physical phenomena. It includes the dynamic growth and detachment of molten droplets, thermal phenomena in the wire, heat transfer from the arc, and the effect of electromagnetic field due to welding current. Because of the complexities associated with these effects, models in the literature [25-57] for prediction of metal transfer are typically simplified and take only those major effects influencing the process under consideration. In this work, an unsteady two-dimensional axisymmetric model is used to investigate droplet evolution, detachment frequency, and the selection of pulse parameters for optimal metal transfer in GMAW. The dynamics of the droplet formation and detachment process are formulated as an incompressible viscous flow with free surfaces. The forces, which significantly influence the metal transfer process, are the gravitational, electromagnetic, and surface tension forces. 


\subsection{Physical Process of Metal Transfer}

Figure 2.1 shows the basic physical process of metal transfer during GMAW. An electric arc is struck between the tip of an electrode, the anode, and the workpiece, the cathode. The consumable electrode is melted under the combined influences of heating

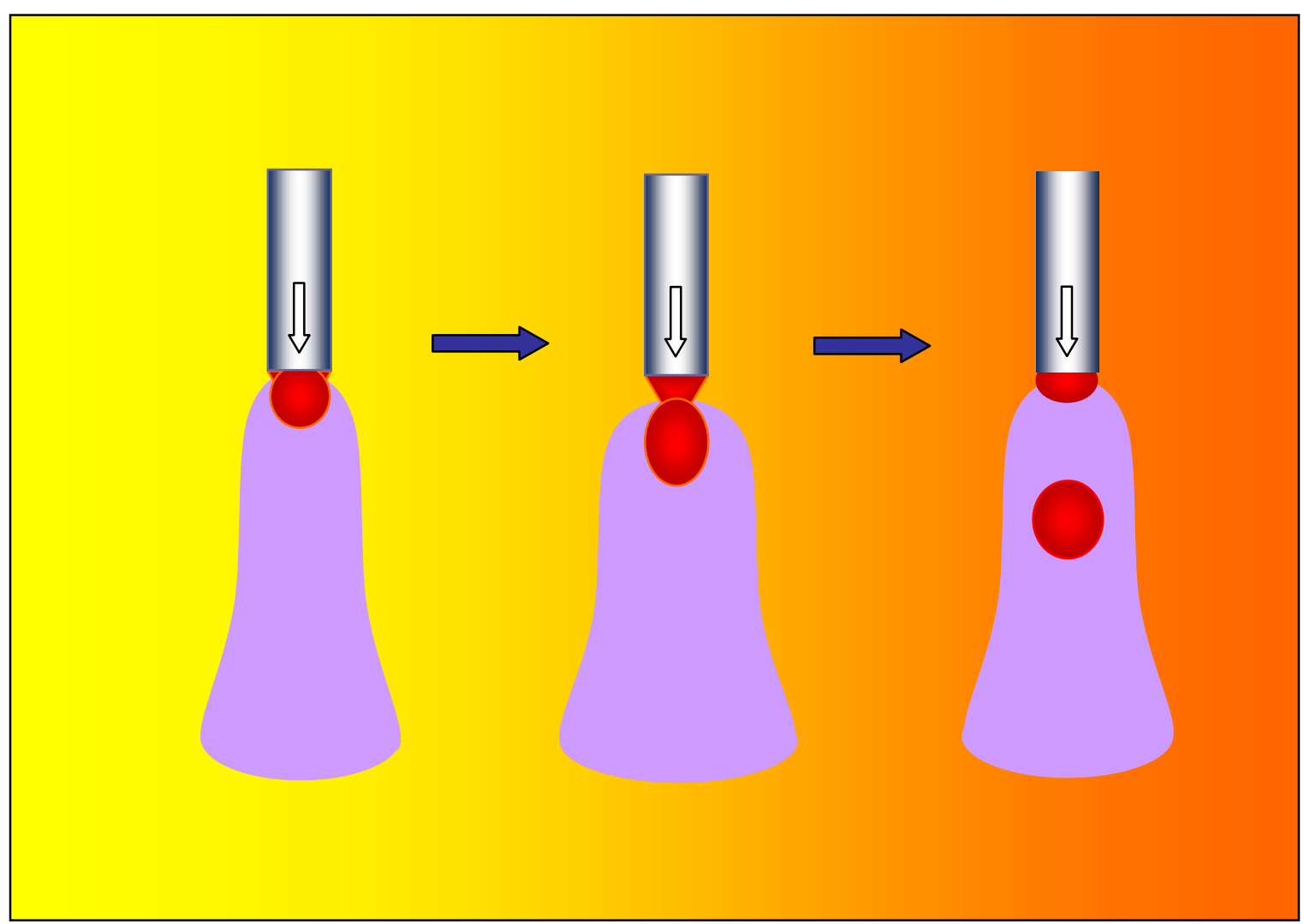

Figure 2.1 The sketch of metal transfer process in GMAW 
produced by welding arc and Joule heating. The molten metal grows at the end of electrode as a pendant droplet. After neck shrinking, the metal droplet breaks from the electrode and transfers downward through the arc into the weld pool. This process is governed by a combination of factors including the balance of forces, thermal phenomena and electromagnetic field. In the present study, thermal phenomena are neglected by assuming the input velocity of molten metal to be the same as the wire feed rate.

In the molten droplet growth and detachment process, the molten droplet experiences the gravitational force, the surface tension force which arises from the free surface, the electromagnetic force which is generated by the interaction of the welding current with its self-induced electromagnetic field, and the plasma drag force which is induced by the surrounding gas ionized in the welding arc. Since plasma drag force has less impact compared with the other forces, it is neglected in the calculation.

The balance of forces determines the droplet profile and detachment frequency. While the gravitational force tends to pull the droplet off and the surface tension force tries to retain it on the tip of electrode, the electromagnetic force is the most potent to accelerate the droplet off the end of the electrode as the welding current increases. The competition between the gravitational, surface tension and electromagnetic forces determines the mode of metal transfer and further influences the welding quality. Large, pendant droplets are grown at low current under the dominance of the gravitational force and the surface tension force in the presence of relatively small electromagnetic forces. The globular transfer occurs with the detachment of droplets having a diameter much 
greater than the diameter of the electrode. As welding current increases, the electromagnetic force becomes significant and accelerates the droplet detachment. The spray transfer occurs with the detachment of small droplets having diameter equal to or less than the diameter of the electrode.

The vast majority of experiments in the literature were performed with mild steel electrodes. Hence, mild steel electrodes are also adopted in this study. The material properties of mild steel are taken from the work of Chio, Yoo and Kim [43]. They are listed in Table 1.

Table 1. Material Properties of the Electrode

\begin{tabular}{ll}
\hline Mass density $\rho$ & $7860 \mathrm{~kg} / \mathrm{m}^{3}$ \\
Kinematic viscosity $v$ & $2.8 \times 10^{-7} \mathrm{~m}^{2} / \mathrm{s}$ \\
Surface tension coefficient $\gamma$ & $1.2-1.8 \mathrm{~N} / \mathrm{m}^{5}$ \\
Electrical Conductivity $\sigma$ & $8.54 \times 10^{5} \mathrm{mho} / \mathrm{m}$ \\
Permeability $\mu$ & $4 \pi \times 10^{-7} \mathrm{H} / \mathrm{m}$ \\
\hline
\end{tabular}

Among these properties, the surface tension coefficient is the most critical because it determines the attaching force - surface tension force. Furthermore, the surface tension coefficient of the molten metal is sensitive to the component of the electrode, the temperature, the shielding gas, etc. For the mild steel used in the present study, it varies approximately in the range of 1.2-1.8 N/m depending on the temperature of the molten steel. For the constant current GMAW, the surface tension coefficient is assumed to be $1.2 \mathrm{~N} / \mathrm{m}$ in the present study. For pulsed current GMAW, the molten droplet temperature 
is relatively low due to the low average current, therefore the surface tension coefficient is assumed to be $1.5 \mathrm{~N} / \mathrm{m}$. A thermal analysis should be incorporated in the model to consider variations of the material properties in the future study.

The following assumptions have also been made concerning the material properties during the metal transfer process:

(1) The physical properties of the material are constant in the same phase, independent of the temperature.

(2) The molten metal is an incompressible Newtonian fluid.

(3) Chemical reaction and metal vaporization are negligible.

\subsection{Modeling of Metal Transfer}

Based upon the above analysis, metal transfer in GMAW is modeled as an unsteady incompressible viscous flow with strong surface tension on free surface. The electromagnetic force significantly influences the metal transfer process. The electric field, which is used to solve the electromagnetic force, is assumed to be quasi-steadystate. An axisymmetric geometrical shape is used to model the shape of molten metal.

Schematic sketches of metal transfer process in GMAW with initial and boundary conditions are shown in Figure 2.2. The following assumptions have been made for the present study: 


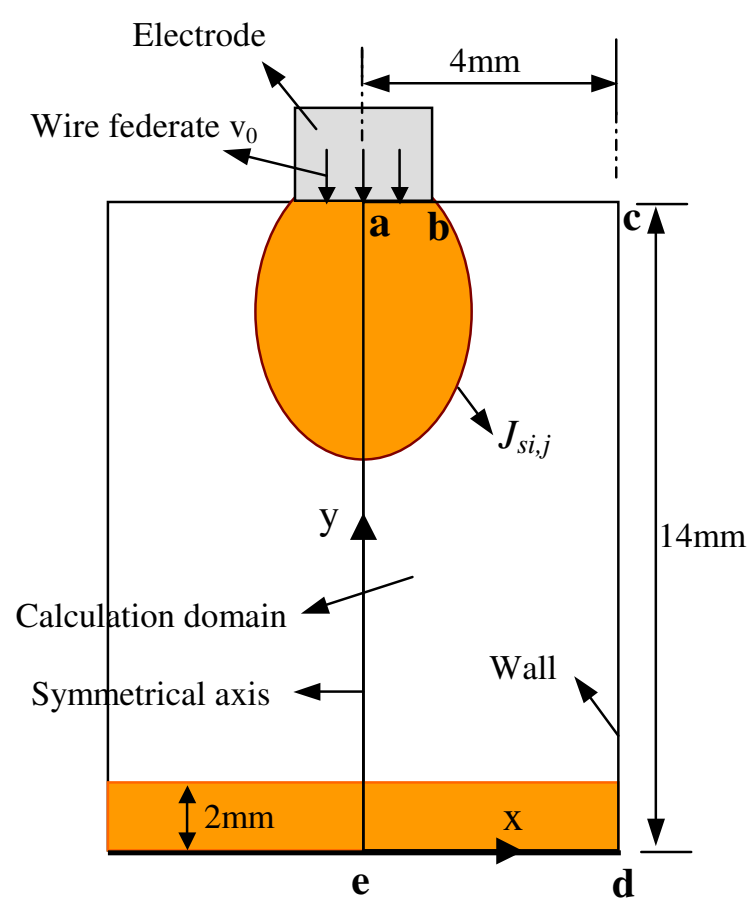

(a)

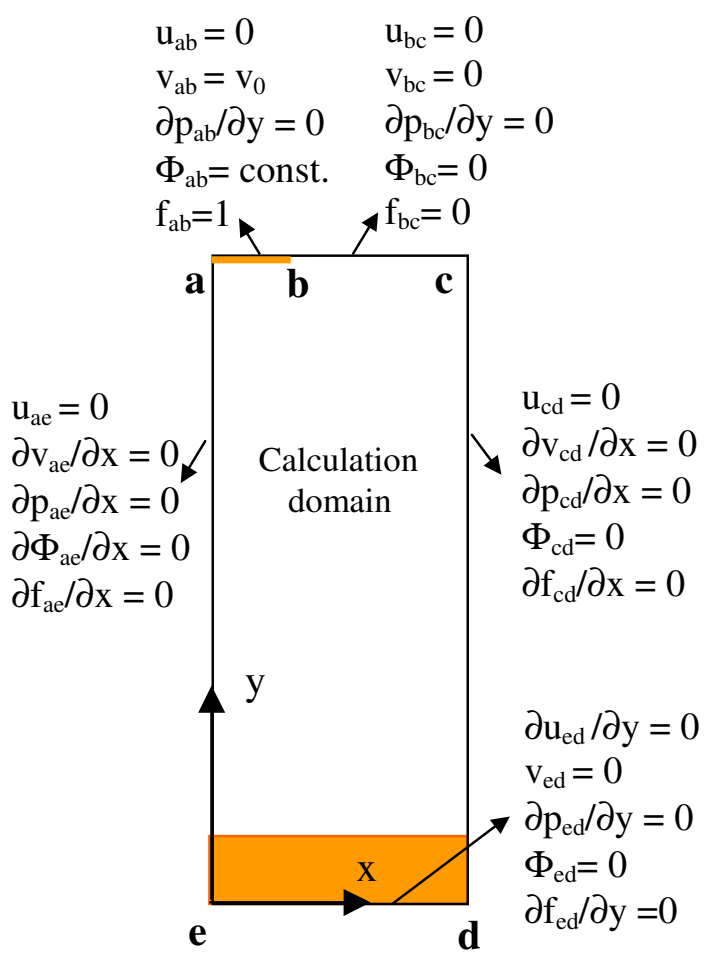

(b)

Figure 2.2 Schematic sketch of metal transfer process in GMAW with initial and boundary conditions: (a) A Schematic of metal transfer process (b) Initial and external boundary conditions

(1) Incompressible laminar fluid flow is assumed;

(2) The input velocity of molten metal is assumed to be the same as the wire feed rate;

(3) The problem is assumed to be axisymmetric. Hence, the calculation domain is taken as one side of centerline; 
(4) Free slip at the solid boundaries;

(5) Momentum transfer from plasma to the droplet is neglected; the velocities of the surrounding gas are specified by setting them to zero;

(6) The effects of pressure variations in the surrounding gas have been neglected by setting the pressure to atmospheric conditions.

The boundary conditions, which are used to determine the distributions of the potential and current density within the droplet, and thus incorporate the influence of the electromagnetic force, are

(1) An isopotential line $(\Phi=0)$ is set at the inlet section;

(2) There is symmetry about the centerline;

(3) The current density on the droplet surface cell (i, j) is $J_{s i, j}$.

Since there are no experimental measurements of the current density on a GMAW droplet surface available in the literature due to the difficulty of making such measurements on the free surface of a metal droplet surrounded by the harsh environment of welding arc, we assumed that current density $J_{s i, j}$ on the droplet surface is distributed as following

$$
J_{s i, j}=c f(i, j)
$$

By considering the current continuity

$$
I=\sum J_{s i, j} S_{i, j}
$$

the current density on the droplet surface cell $(i, j)$ becomes 


$$
J_{s i, j}=I \cdot f(i, j) / \sum_{n}\left(S_{i, j} \cdot f(i, j)\right)
$$

where $I$ is the welding current, and $S_{i, j}$ is the surface area of the free surface cell $(i, j)$. $f(i, j)$ is the distribution function, which has to be assumed. The two kinds of current density distribution on the droplet surface assumed in the previous study by Chio, Yoo and Kim [43] are as follows:

$$
\begin{array}{ll}
\text { Uniform current density distribution: } & f(i, j)=1 \\
\text { Linear current density distribution: } & f(i, j)=z_{j}
\end{array}
$$

where $z_{\mathrm{j}}$ represents the distance between the free surface cell $(\mathrm{i}, \mathrm{j})$ and the solid-liquid interface of the electrode. It was found that the current density distribution on the droplet surface had significant effects on the molten droplet profile and size. The calculated results were in broad agreement with the experimental data and suggested that the assumption of the linear current density predicted the experimental results more accurately than the uniform current density. However, the transition current was not captured using either of these current density distribution models.

In the present work, a Gaussian current density distribution on the droplet surface is proposed:

$$
\begin{aligned}
& f(i, j)=\frac{1}{\sqrt{2 \pi}} \exp \left(-\xi_{i, j}^{2} / 2\right) \\
& \xi_{i, j}=\frac{X_{i, j}}{D}
\end{aligned}
$$


where $X_{i, j}$ is the arc (curve) length on the droplet surface between the lowest point on the droplet and the free surface cell $(\mathrm{i}, \mathrm{j})$, and $D$ is diameter of the electrode when the welding current is constant. The assumption is proposed based on the current density distribution over the surface of the underlying workpiece, for which a radially symmetric Gaussian distribution has been detected by previous experiments $[6,21]$ and has been adopted frequently in the literature.

Copyright $\odot$ Ge Wang 2007 


\section{Chapter 3}

\section{Numerical Schemes}

Our numerical program is developed based on RIPPLE - a computer program for solving incompressible flows with free surfaces provided by Los Alamos National Laboratory, by adding electromagnetic field calculation. The numerical schemes employed are based on a finite-difference solution of a coupled set of partial differential equations governing unsteady incompressible fluid flow with surface tension on the free surface [60] and influence coming from the electromagnetic force. The two-step projection method [66] is the basic algorithm for solving this set of partial differential equations, with the pressure Poisson equation (PPE) solved by a robust incomplete Cholesky conjugate gradient (ICCG) technique. Free surfaces are captured by the volume of fluid (VOF) method [61]. Surface tension of free surfaces is modeled as a localized

volume force derived from the continuum surface force (CSF) model [62]. The electromagnetic force is calculated based on assumption of quasi-steady-state electric field and Gaussian current density distribution over the free surface. A boundary condition must be enforced to a transient, irregular surface - the free surface. 


\subsection{Governing Equations}

In order to simplify the numerical model, the physical process of metal transfer is assumed to be axisymmetric, and the material properties are assumed to be constant. The motion of fluid within the droplet is governed by the two dimensional, incompressible Navier-Stokes equations (continuity and momentum equations) in Cartesian or cylindrical $(x=r, y=z)$ coordinates:

$$
\begin{aligned}
& \nabla \cdot \vec{v}=0 \\
& \rho \frac{D \vec{v}}{D t}=-\nabla p+\nabla \cdot \tau+\vec{F}_{b}
\end{aligned}
$$

In the above, $\vec{v}$ is the velocity, $\rho$ is the fluid density, $p$ is the scalar pressure, $\vec{F}_{b}$ is the body force, which includes the gravitational force, surface tension force and the electromagnetic force, and $\tau$ is the viscous stress tensor. The element of viscous stress tensor $\tau_{x y}$ is

$$
\tau_{x y}=\mu\left(\frac{\partial v_{x}}{\partial x_{y}}+\frac{\partial v_{y}}{\partial x_{x}}\right)
$$

where $\mu$ is the dynamic viscosity coefficient of the fluid. 


\subsection{Numerical Modeling and Solution for Governing Equations}

When obtaining numerical solution of the governing equations, some general steps should be followed: 1. Generate a layout of the finite difference mesh in the calculation domain. 2. Formulate the discretization form of the governing equation. 3. Set initial conditions according to the physical model. 4. Add boundary condition according to the physical model. 5. Solve the system of algebraic equations. Figure 3.1 shows a flow diagram of those steps.

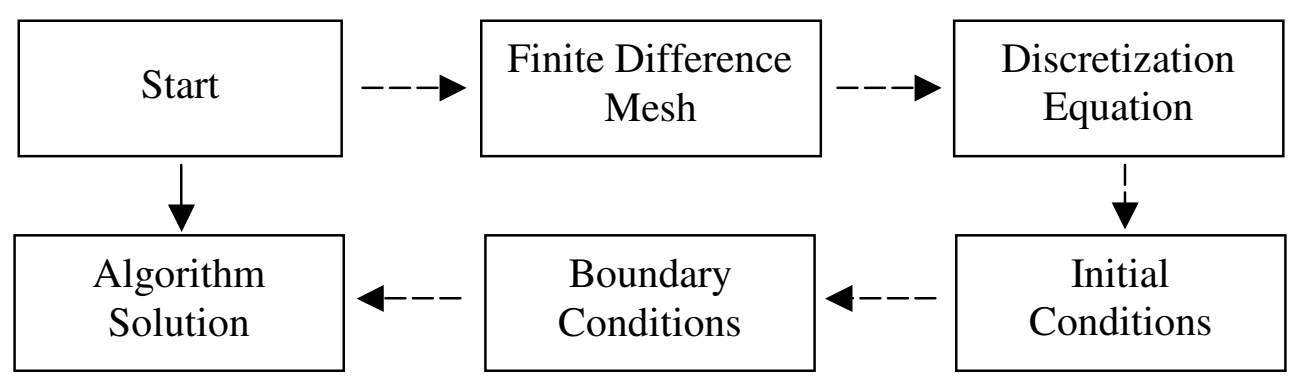

Figure 3.1 Flow Diagram of Numerical Solution

\subsubsection{Mesh Layout}

The calculation domain is partitioned into a rectilinear mesh, which is composed of orthogonal cells with variable width and height $\delta x_{i}, \delta y_{j}$ for a cell centered at the point $\left(x_{i}, y_{j}\right)$. The computational mesh is constructed from a number of sub-meshes with each sub-mesh built by quadratically expanding cell spacing. Arbitrary variable cell spacing is achieved by properly choosing the specified variables. 
Figure 3.2 shows layout of variables $p, \rho$ and $\vec{v}$ on a cell centered at $\left(x_{i}, y_{j}\right)$.

The pressure $p_{i, j}$ and density $\rho_{i, j}$ are located at the cell center $\left(x_{i}, y_{j}\right)$. The components of velocity are set at cell faces with $x$ component $u_{i+1 / 2, j}$ at position $\left(x_{i+1 / 2}, y_{j}\right)$ and $y$ component $v_{i, j+1 / 2}$ at position $\left(x_{i}, y_{j+1 / 2}\right)$. The control volumes for mass and momentum are marked in the figure with the mass control volume centered at $\left(x_{i}, y_{j}\right)$, the $x$ momentum control volume centered at $\left(x_{i+1 / 2}, y_{j-1}\right)$ and the $y$-momentum control volume centered at $\left(x_{i-1}, y_{j+1 / 2}\right)$.

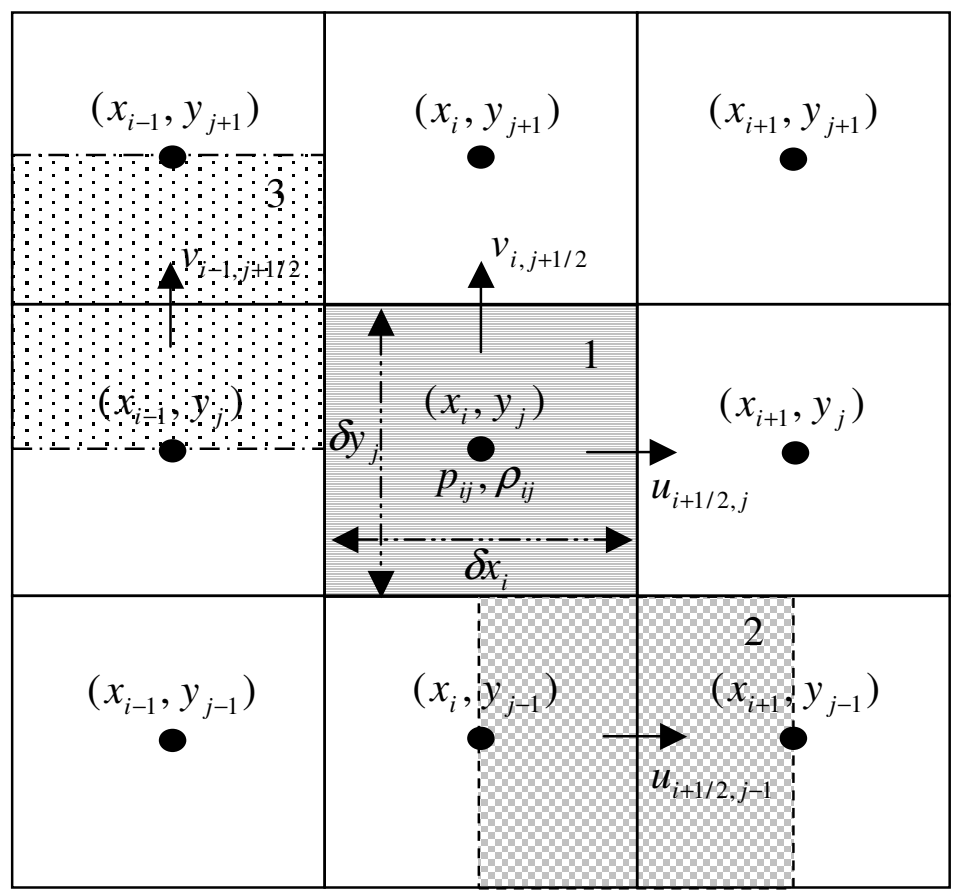

Figure 3.2 Control volumes 
The staggered arrangement of pressure and velocities results in second order accurate, central differences. The key feature of this staggered grid arrangement is that the mass flux across any face of the fluid volume over which continuity is to be satisfied is driven by a pressure difference evaluated with pressures at nodes straddling the mass flux interfaces. This layout tends to prevent inappropriate decoupling between the pressure and velocity fields, which yields a false numerical solution. The false solution can be highlighted by a checker-board distribution for the pressure field as shown in Figure 3.3. It is apparently not a zero-pressure gradient field in physics. However, a false zero-gradient pressure field $\left.\frac{\partial p}{\partial x}\right|_{\mathrm{P}}=\frac{p_{\mathrm{E}}-p_{\mathrm{W}}}{\Delta x_{\mathrm{EW}}}=0$ is obtained numerically when a nonstaggered grid arrangement of pressure and velocities is applied.

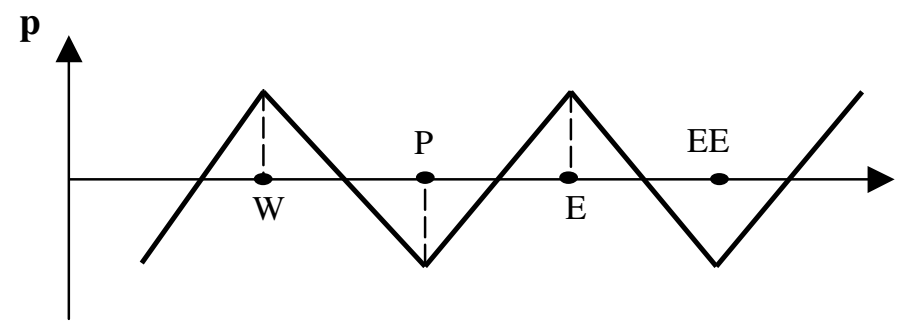

Figure 3.3 Checker-board distribution for pressure

\subsubsection{The Discretization of Governing Equations and Algorithm Solution}

Given a partial differential equation and a finite difference mesh, there are two primary approaches to develop finite difference equations: the point approach and the control volume approach. The point approach develops a finite difference approximation 
for $\left(\frac{\partial \phi}{\partial x}\right)_{i j}$ by using the Taylor series expansions. The discretization is derived in a mechanical way while the physical laws used in deriving the partial differential equations are not scrutinized. The control volume approach forces the finite difference equations to follow the physical laws or conservation principles that the partial differential equations represent. Hence, the control volume approach is adopted to build finite difference equations here. Using the control volumes shown in Figure 3.2, governing equations 3.1 and 3.2 are discretized as first order accurate in time and second order accurate in space. The details of the governing equations discretization will be given in the following subsections.

\section{a. The Two-step Projection Method}

The two-step projection method [66] is used as the basic algorithm to solve the set of partial differential governing equations here. A time discretization form of the momentum equation (3.2) is

$$
\frac{\vec{v}^{n+1}-\vec{v}^{n}}{\Delta t}=-\left(\vec{v}^{n} \cdot \nabla\right) \vec{v}^{n}-\frac{1}{\rho^{n}} \nabla p^{n+1}+\frac{1}{\rho^{n}} \nabla \cdot \tau^{n}+\frac{1}{\rho^{n}} \vec{F}_{b}^{n}
$$

The basic scheme for the two-step projection method is to break the computation of the governing equations for unsteady incompressible flow (3.1) and (3.4) into two steps.

$$
\text { Step 1: } \quad \frac{\vec{v}^{*}-\vec{v}^{n}}{\Delta t}=-\left(\vec{v}^{n} \cdot \nabla\right) \vec{v}^{n}+\frac{1}{\rho^{n}} \nabla \cdot \tau^{n}+\frac{1}{\rho^{n}} \vec{F}_{b}^{n}
$$




$$
\begin{array}{r}
\text { Step } \left.2: \begin{array}{r}
\frac{\vec{v}^{n+1}-\vec{v}^{*}}{\Delta t}=-\frac{1}{\rho^{n}} \nabla p^{n+1} \\
\nabla \cdot \vec{v}^{n+1}=0
\end{array}\right\} \\
\Rightarrow \nabla \cdot\left(\frac{1}{\rho^{n}} \nabla p^{n+1}\right)=\frac{\nabla \cdot \vec{v}^{*}}{\Delta t}
\end{array}
$$

In the first step, a velocity field $\vec{v}^{*}$ is computed from diffusion, advection and body forces, i.e., neglecting the influence from the pressure gradient. In the second step, the velocity field is changed under the influence of pressure gradient only. Since the velocity field must satisfy the continuity equation (3.1) as well, one Poisson equation (3.7) is obtained for solving the pressure field. This Poisson equation is referred to as the pressure Poisson equation (PPE). The superscript $n$ represents the value of variable when time is $t^{n}$. The time step is variable with its value $\Delta t_{k}$ at the $\mathrm{k}^{\text {th }}$ cycle $(\mathrm{k}=0,1,2 \ldots)$. After $\mathrm{n}$ cycles, the time is $t^{n}=\sum_{k=0}^{n-1} \Delta t_{k}$.

b. Advection

The finite difference form of advection terms in the $\mathrm{x}$ - and $\mathrm{y}$-directions are derived using the momentum control volumes shown in Figure 3.2.

For the advection term in the $\mathrm{x}$-direction, it is calculated at $(i+1 / 2, j)$,

$$
(\vec{v} \cdot \nabla) u=u_{i+1 / 2, j}\left(\frac{\partial u}{\partial x}\right)_{i+1 / 2, j}+v_{i+1 / 2, j}\left(\frac{\partial u}{\partial y}\right)_{i+1 / 2, j}
$$

where 


$$
\begin{gathered}
v_{i+1 / 2, j}=\frac{\delta x_{i} v_{i+1, j}+\delta x_{i+1} v_{i, j}}{\delta x_{i}+\delta x_{i+1}} \\
=\frac{\delta x_{i}\left(v_{i+1, j-1 / 2}+v_{i+1, j+1 / 2}\right) / 2+\delta x_{i+1}\left(v_{i, j-1 / 2}+v_{i, j+1 / 2}\right) / 2}{\delta x_{i}+\delta x_{i+1}} \\
\left(\frac{\partial u}{\partial x}\right)_{i+1 / 2, j}=\frac{\delta x_{i+1}}{\delta x_{\alpha}}\left(1+\alpha s_{u}\right)\left(\frac{\partial u}{\partial x}\right)_{i, j}+\frac{\delta x_{i}}{\delta x_{\alpha}}\left(1-\alpha s_{u}\right)\left(\frac{\partial u}{\partial x}\right)_{i+1, j} \\
=\frac{\delta x_{i+1}}{\delta x_{\alpha}}\left(1+\alpha s_{u}\right)\left(\frac{u_{i+1 / 2, j}-u_{i-1 / 2, j}}{\delta x_{i}}\right)+\frac{\delta x_{i}}{\delta x_{\alpha}}\left(1-\alpha s_{u}\right)\left(\frac{u_{i+1+1 / 2, j}-u_{i+1 / 2, j}}{\delta x_{i+1}}\right) \\
\left(\begin{array}{c}
\left.\frac{\partial u}{\partial y}\right)_{i+1 / 2, j} \\
=\frac{\delta y_{j+1 / 2}}{\delta y_{\alpha}}\left(1+\alpha s_{v}\right)\left(\frac{\partial u}{\partial y}\right)_{i+1 / 2, j-1 / 2} \\
\delta y_{j+1 / 2} \\
\delta y_{\alpha}\left(1+\alpha s_{v}\right)\left(\frac{u_{i+1 / 2, j}}{\delta y_{\alpha}}\left(1-\alpha s_{v}\right)\left(\frac{\partial u}{\partial y}\right)_{i+1 / 2, j+1 / 2}\right. \\
\left.\delta y_{j-1 / 2}\right)+\frac{\delta y_{j-1 / 2}}{\delta y_{\alpha}}\left(1-\alpha s_{v}\right)\left(\frac{u_{i+1 / 2, j+1}-u_{i+1 / 2, j}}{\delta y_{j+1 / 2}}\right) \\
\delta y_{\alpha}=\delta y_{j+1 / 2}+\delta y_{j-1 / 2}+\alpha s_{v}\left(\delta y_{j+1 / 2}-\delta y_{j-1 / 2}\right), \\
s_{v}=s i g n\left(u_{i+1 / 2, j}\right)
\end{array}\right.
\end{gathered}
$$

Similarly, the advection term in the direction of $y$-axis is given by

$$
(\vec{v} \cdot \nabla) v=u_{i, j+1 / 2}\left(\frac{\partial v}{\partial x}\right)_{i, j+1 / 2}+v_{i, j+1 / 2}\left(\frac{\partial v}{\partial y}\right)_{i, j+1 / 2}
$$

where

$$
\begin{gathered}
u_{i, j+1 / 2}=\frac{\delta y_{j} u_{i, j+1}+\delta y_{j+1} u_{i, j}}{\delta y_{j}+\delta y_{j+1}} \\
=\frac{\delta y_{j}\left(u_{i-1 / 2, j+1}+u_{i+1 / 2, j+1}\right) / 2+\delta y_{j+1}\left(u_{i-1 / 2, j}+u_{i+1 / 2, j}\right) / 2}{\delta y_{j}+\delta y_{j+1}} \\
\left(\frac{\partial v}{\partial x}\right)_{i, j+1 / 2}=\frac{\delta x_{i+1 / 2}}{\delta x_{\alpha}{ }^{\prime}}\left(1+\alpha s_{u}{ }^{\prime}\right)\left(\frac{\partial v}{\partial x}\right)_{i-1 / 2, j+1 / 2}+\frac{\delta x_{i-1 / 2}}{\delta x_{\alpha}}\left(1-\alpha s_{u}{ }^{\prime}\right)\left(\frac{\partial v}{\partial x}\right)_{i+1 / 2, j+1 / 2} \\
=\frac{\delta x_{i+1 / 2}}{\delta x_{\alpha}{ }^{\prime}}\left(1+\alpha s_{u}{ }^{\prime}\right)\left(\frac{v_{i, j+1 / 2}-v_{i-1, j+1 / 2}}{\delta x_{i-1 / 2}}\right)+\frac{\delta x_{i-1 / 2}}{\delta x_{\alpha}}\left(1-\alpha s_{u}{ }^{\prime}\right)\left(\frac{v_{i+1, j+1 / 2}-v_{i, j+1 / 2}}{\delta x_{i+1 / 2}}\right)
\end{gathered}
$$




$$
\begin{aligned}
& \left(\frac{\partial v}{\partial y}\right)_{i, j+1 / 2}=\frac{\delta y_{j+1}}{\delta y_{\alpha}^{\prime}}\left(1+\alpha s_{v}{ }^{\prime}\right)\left(\frac{\partial v}{\partial y}\right)_{i, j}+\frac{\delta y_{j}}{\delta y_{\alpha}^{\prime}}\left(1-\alpha s_{v}{ }^{\prime}\right)\left(\frac{\partial v}{\partial y}\right)_{i, j+1} \\
& =\frac{\delta y_{j+1}}{\delta y_{\alpha}^{\prime}}\left(1+\alpha s_{v}{ }^{\prime}\right)\left(\frac{v_{i, j+1 / 2}-v_{i, j-1 / 2}}{\delta y_{j}}\right)+\frac{\delta y_{j}}{\delta y_{\alpha}^{\prime}}\left(1-\alpha s_{v}{ }^{\prime}\right)\left(\frac{v_{i, j+1+1 / 2}-v_{i, j+1 / 2}}{\delta y_{j+1}}\right) \\
& \delta x_{\alpha}^{\prime}=\delta x_{i-1 / 2}+\delta x_{i+1 / 2}+\alpha s_{u}{ }^{\prime}\left(\delta x_{i+1 / 2}-\delta x_{i-1 / 2}\right), \\
& \delta y_{\alpha}^{\prime}=\delta y_{j}+\delta y_{j+1}+\alpha s_{v}{ }^{\prime}\left(\delta y_{j+1}-\delta y_{j}\right), \quad s_{u}{ }^{\prime}=\operatorname{sign}\left(u_{i, j+1 / 2}\right)
\end{aligned}
$$

The variable coefficient $0 \leq \alpha \leq 1$ controls the finite difference representation of the advection terms with the linear combination of donor cell difference and centered difference.

$$
\alpha=\left\{\begin{aligned}
0 & \text { Centered Difference } \\
0<\alpha<1 & \text { Interpolated Difference } \\
1 & \text { Donor Cell Difference }
\end{aligned}\right.
$$

A value of $\alpha$ greater than $1 / 2$ is recommended to offset the unstable negative diffusion brought by the first order forward time difference.

c. Viscosity

The viscous term is given by:

$$
\nabla \cdot \tau=\left[\frac{1}{r^{\delta}} \frac{\partial}{\partial x}\left(r^{\delta} \tau_{x x}\right)+\frac{\partial \tau_{y x}}{\partial y}\right] \vec{i}+\left[\frac{1}{r^{\delta}} \frac{\partial}{\partial x}\left(r^{\delta} \tau_{x y}\right)+\frac{\partial \tau_{y y}}{\partial y}\right] \vec{j}
$$

where superscript $\delta$ is a constant equal to 1 in cylindrical and 0 in Cartesian coordinates. For $x$-momentum control volume, the viscous term is located at $(i+1 / 2, j)$ : 


$$
\begin{aligned}
\left(\frac{1}{r^{\delta}} \frac{\partial}{\partial x}\left(r^{\delta} \tau_{x x}\right)+\frac{\partial \tau_{y x}}{\partial y}\right)_{i+1 / 2, j} & =\frac{1}{r_{i+1 / 2}^{\delta} \delta x_{i+1 / 2}}\left(r_{i+1}^{\delta} \tau_{x x i+1, j}-r_{i}^{\delta} \tau_{x x i, j}\right) \\
& +\frac{1}{\delta y_{j}}\left(\tau_{y x i+1 / 2, j+1 / 2}-\tau_{y x i+1 / 2, j-1 / 2}\right)
\end{aligned}
$$

For $y$-momentum, the viscous term is calculated at $(i, j+1 / 2)$ :

$$
\begin{aligned}
\left(\frac{1}{r^{\delta}} \frac{\partial}{\partial x}\left(r^{\delta} \tau_{x y}\right)+\frac{\partial \tau_{y y}}{\partial y}\right)_{i, j+1 / 2}= & \frac{1}{r_{i}^{\delta} \delta x_{i}}\left(r_{i+1 / 2}^{\delta} \tau_{x y i+1 / 2, j+1 / 2}-r_{i-1 / 2}^{\delta} \tau_{x y i-1 / 2, j+1 / 2}\right) \\
& +\frac{1}{\delta y_{j+1 / 2}}\left(\tau_{y y i, j+1}-\tau_{y y i, j}\right)
\end{aligned}
$$

where

$$
\begin{aligned}
& \tau_{x x i, j}=2 \mu\left(\frac{u_{i+1 / 2, j}-u_{i-1 / 2, j}}{\delta x_{i}}\right) \\
& \tau_{y y i, j}=2 \mu\left(\frac{v_{i, j+1 / 2}-v_{i, j-1 / 2}}{\delta y_{j}}\right) \\
& \tau_{x y i+1 / 2, j+1 / 2}=\mu\left(\frac{u_{i+1 / 2, j+1}-u_{i+1 / 2, j}}{\delta y_{j+1 / 2}}+\frac{v_{i+1, j+1 / 2}-v_{i, j+1 / 2}}{\delta x_{i+1 / 2}}\right)
\end{aligned}
$$

\section{d. Poisson Equation}

For simplification, the diffusion term in Poisson equation (3.7) is mapped from the physical coordinates $(x, y)$ into natural coordinates $(\zeta, \eta)$, which are linear quadrilaterals of unit length: 


$$
\begin{aligned}
\nabla \cdot\left(\frac{1}{\rho} \nabla p^{n+1}\right)= & \frac{1}{J r^{\delta}}\left[\left(\frac{r^{\delta} \alpha}{\rho}\right) p_{\xi}^{n+1}-\left(\frac{r^{\delta} \beta}{\rho}\right) p_{\eta}^{n+1}\right]_{\xi} \\
& +\frac{1}{J r^{\delta}}\left[\left(\frac{r^{\delta} \gamma}{\rho}\right) p_{\eta}^{n+1}-\left(\frac{r^{\delta} \beta}{\rho}\right) p_{\xi}^{n+1}\right]_{\eta}
\end{aligned}
$$

where

$$
\begin{aligned}
& \frac{\partial()}{\partial \xi}=()_{\xi}, \quad \frac{\partial()}{\partial \eta}=()_{\eta} \\
& J=\frac{\partial(x, y)}{\partial(\xi, \eta)}=x_{\xi} y_{\eta}-x_{\eta} y_{\xi} \\
& \alpha=\frac{1}{J}\left(x_{\eta}^{2}+y_{\eta}^{2}\right), \quad \beta=\frac{1}{J}\left(x_{\xi} x_{\eta}+y_{\xi} y_{\eta}\right), \quad \gamma=\frac{1}{J}\left(x_{\xi}^{2}+y_{\xi}^{2}\right)
\end{aligned}
$$

The superscript $\delta$ on the radius $r$ is a constant equal to 1 in cylindrical and 0 in Cartesian coordinates. Since the mesh is composed of orthogonal cells centered at the point $\left(x_{i}, y_{j}\right)$, the cross derivatives are zero:

$$
\begin{array}{ll}
x_{\xi i, j}=x_{i}-x_{i-1}, & x_{\eta i, j}=0 \\
y_{\xi i, j}=0, & y_{\eta i, j}=y_{j}-y_{j-1}
\end{array}
$$

Hence:

$$
\begin{aligned}
& \alpha_{i, j}=\frac{y_{\eta i, j}^{2}}{J_{i, j}}, \quad \beta_{i, j}=0, \quad \gamma_{i, j}=\frac{x_{\xi i, j}^{2}}{J_{i, j}} \\
& J_{i, j}=x_{\xi i, j} y_{\eta i, j}
\end{aligned}
$$


For the control volumes shown in Figure 3.2, the pressure $p_{i, j}$ is located at center of cell $(i, j)$.

$$
\begin{aligned}
& {\left[\left(\frac{r^{\delta} \alpha}{\rho}\right) p_{\xi}^{n+1}-\left(\frac{r^{\delta} \beta}{\rho}\right) p_{\eta}^{n+1}\right]_{\xi i, j}=\left[\left(\frac{r^{\delta} \alpha}{\rho}\right) p_{\xi}^{n+1}-\left(\frac{r^{\delta} \beta}{\rho}\right)_{\eta}^{0} p_{i+1 / 2, j}^{n+1}\right.} \\
& -\left[\left(\frac{r^{\delta} \alpha}{\rho}\right) p_{\xi}^{n+1}-\left(\frac{r^{\delta} \beta}{\rho}\right)^{0} p_{\eta}^{n+1}\right]_{i-1 / 2, j} \\
& =\left(\frac{r^{\delta} \alpha}{\rho}\right)_{i+1 / 2, j} p_{\xi i+1 / 2, j}^{n+1}-\left(\frac{r^{\delta} \alpha}{\rho}\right)_{i-1 / 2, j} p_{\xi i-1 / 2, j}^{n+1} \\
& {\left[\left(\frac{r^{\delta} \gamma}{\rho}\right) p_{\eta}^{n+1}-\left(\frac{r^{\delta} \beta}{\rho}\right) p_{\xi}^{n+1}\right]_{\eta i, j}=\left[\left(\frac{r^{\delta} \gamma}{\rho}\right) p_{\eta}^{n+1}-\left(\frac{r^{\delta} \beta}{\rho}\right)^{0} p_{\xi}^{n+1}\right]_{i, j+1 / 2}} \\
& -\left[\left(\frac{r^{\delta} \gamma}{\rho}\right) p_{\eta}^{n+1}-\left(\frac{r^{\delta} \beta}{\rho}\right)^{0} p_{\xi}^{n+1}\right]_{i, j-1 / 2} \\
& =\left(\frac{r^{\delta} \gamma}{\rho}\right)_{i, j+1 / 2} p_{\eta i, j+1 / 2}^{n+1}-\left(\frac{r^{\delta} \gamma}{\rho}\right)_{i, j-1 / 2} p_{\eta i, j-1 / 2}^{n+1}
\end{aligned}
$$

where

$$
\begin{aligned}
& p_{\xi i+1 / 2, j}^{n+1}=p_{i+1, j}^{n+1}-p_{i, j}^{n+1} \\
& p_{\eta i, j+1 / 2}^{n+1}=p_{i, j+1}^{n+1}-p_{i, j}^{n+1}
\end{aligned}
$$

Substituting the above equations into the Poisson equation (3.7), a finite discretization equation can be written as follows:

$$
\begin{aligned}
& A_{i, j} p_{i, j}^{n+1}+B_{i, j} p_{i+1, j}^{n+1}+C_{i, j} p_{i, j+1}^{n+1}+D_{i, j} p_{i-1, j}^{n+1}+E_{i, j} p_{i, j-1}^{n+1}=S_{i, j} \\
& A_{i, j}=\left(r^{\delta} \frac{\alpha}{\rho}\right)_{i+1 / 2, j}+\left(r^{\delta} \frac{\alpha}{\rho}\right)_{i-1 / 2, j}+\left(r^{\delta} \frac{\gamma}{\rho}\right)_{i, j+1 / 2}+\left(r^{\delta} \frac{\gamma}{\rho}\right)_{i, j-1 / 2} \\
& B_{i, j}=-\left(r^{\delta} \frac{\alpha}{\rho}\right)_{i+1 / 2, j}, \quad C_{i, j}=-\left(r^{\delta} \frac{\gamma}{\rho}\right)_{i, j+1 / 2}
\end{aligned}
$$




$$
\begin{aligned}
D_{i, j} & =-\left(r^{\delta} \frac{\alpha}{\rho}\right)_{i-1 / 2, j}, \quad E_{i, j}=-\left(r^{\delta} \frac{\gamma}{\rho}\right)_{i, j-1 / 2} \\
S_{i, j} & =-\frac{r_{i}^{\delta} J_{i, j}}{\delta t}\left(\nabla \cdot \vec{v}^{*}\right)_{i, j} \\
& =-\frac{r_{i}^{\delta} J_{i, j}}{\delta t}\left(\frac{r_{i+1 / 2}^{\delta} u_{i+1 / 2, j}^{*}-r_{i-1 / 2}^{\delta} u_{i-1 / 2, j}^{*}}{r_{i}^{\delta} \delta x_{i}}\right)-\frac{r_{i}^{\delta} J_{i, j}}{\delta t}\left(\frac{v_{i, j+1 / 2}^{*}-v_{i, j-1 / 2}^{*}}{\delta y_{j}}\right)
\end{aligned}
$$

The above finite difference equations can be solved by solving a matrix equation:

$$
\begin{aligned}
& \mathrm{MP}^{n+1}=\mathrm{S} \\
& \mathbf{P}^{n+1}=\left(\begin{array}{c}
p_{1}^{n+1} \\
\vdots \\
p_{k}^{n+1}
\end{array}\right), \quad \mathbf{S}=\left(\begin{array}{c}
S_{1} \\
\vdots \\
S_{k}
\end{array}\right),
\end{aligned}
$$

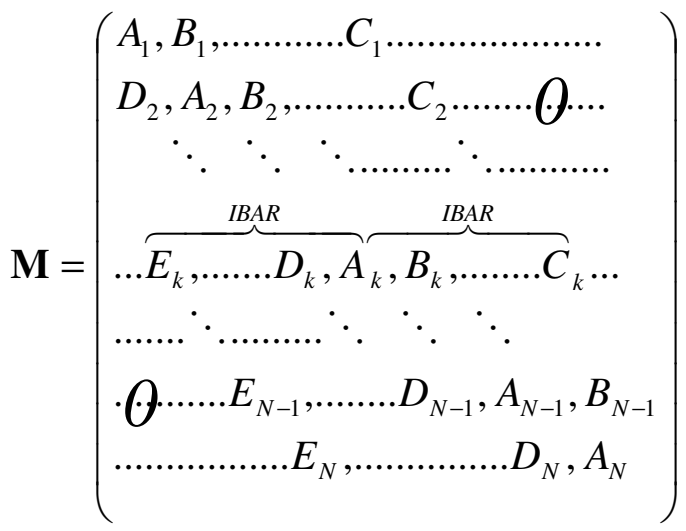

where $k=(j-2) \mathrm{IBAR}+i-1$ within range of $1 \leq k \leq \mathrm{IBAR} * \mathrm{JBAR}$. IBAR and JBAR are the number of real cells in the $\mathrm{x}$ and $\mathrm{y}$ directions respectively.

Since Matrix $\mathrm{M}$ is symmetric, positive definite, sparse, banded and diagonally dominant, an incomplete Cholesky conjugate gradient (ICCG) matrix solution method is used to solve the matrix equation (3.40) and obtain the solution for the pressure field. 


\subsection{Tracking the Free Surface}

The transient shape of the droplet is calculated using the fractional volume of fluid (VOF) method, which was pioneered by Hirt and Nichol. This method has been proved to be more flexible and efficient than other methods for treating problems with a free surface. A detailed discussion of this method can be found in the literature [61]. Its main features are discussed briefly below.

Free surfaces are modeled by means of a scalar function $F(\vec{x}, \mathrm{t})$. The value of $F$ represents the fractional volume of the cell occupied by fluid. A unit value of $F$ represents a cell occupied by fluid, while a zero value of $F$ corresponds to an empty cell. A cell with $F$ value between zero and one contains a free surface.

Hence,

$$
F(\vec{x}, t)=\left\{\begin{array}{rc}
1 & \text { in the fluid } \\
>0,<1 & \text { at the free surface } \\
0 & \text { at the void }
\end{array}\right.
$$

For incompressible flow, the VOF function might be regarded as the normalization $F(\vec{x}, t)=\rho(\vec{x}, t) / \rho_{f}$, where $\rho_{f}$ is the constant fluid density. The discontinuity in $F$ is a Lagrangian invariant, which is governed by the partial differential equation:

$$
\frac{d F}{d t}=\frac{\partial F}{\partial t}+(\vec{v} \cdot \nabla) F=0
$$


The discretization form of the above equation contains terms of $F$ fluxes through the faces of the computational cells. The VOF method is based on the use of reconstructed free surface and a donor-acceptor differencing approximation to calculate fluxes of fractional fluid volume $F$ advected through the cell faces at the free surface. The free surface is reconstructed either horizontally or vertically in a surface cell, depending upon its slope obtained from the value of $F$ in neighboring cells. The position of a free surface is also calculated based upon the value of $F$. A donor-acceptor differencing is identified by the direction of the velocity.

From equation 3.43, the partial time derivative of $F$ satisfies

$$
\left(\frac{\partial F}{\partial t}\right)=-(\vec{v} \cdot \nabla) F
$$

For an incompressible fluid, it yields the equation

$$
\left(\frac{\partial F}{\partial t}\right)=-(\vec{v} \cdot \nabla) F-(\nabla \cdot \vec{v}) F=-\nabla \cdot(\vec{v} F)
$$

The change for $F$ in a cell equals to the amount of fractional fluid volume fluxed across the cell faces. It is still an acceptable approximation even when the fluid is slightly compressible. Hence,

$$
F^{n+1}=F^{n}-\Delta t \nabla \cdot\left(\vec{v}^{n+1} F^{n}\right)
$$

Equation (3.46) is discretized by integrating over a computational cell shown in Figure 3.4 


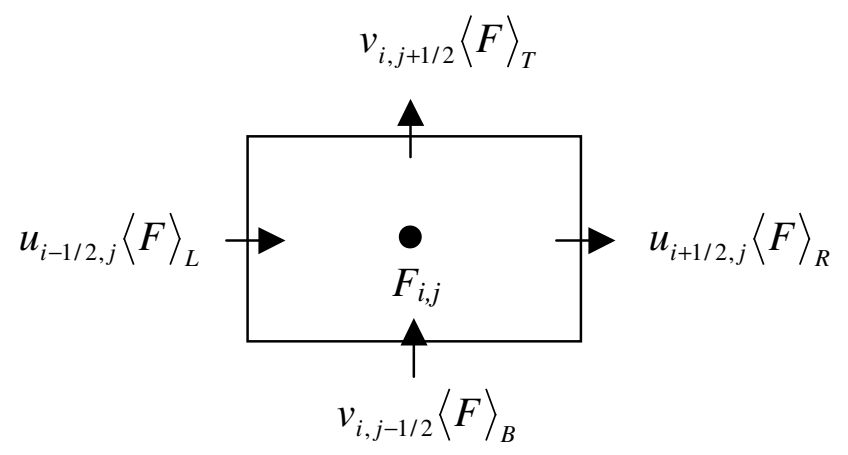

Figure 3.4 Control Volume for VOF function

Giving

$$
\begin{aligned}
F_{i, j}^{n+1}= & F_{i, j}^{n}-\frac{\Delta t}{r_{i}^{\delta} \delta x_{i}}\left[r_{i+1 / 2}^{\delta} u_{i+1 / 2, j}^{n+1}\langle F\rangle_{R}-r_{i-1 / 2}^{\delta} u_{i-1 / 2, j}^{n+1}\langle F\rangle_{L}\right] \\
& -\frac{\Delta t}{\delta y_{j}}\left[v_{i, j+1 / 2}^{n+1}\langle F\rangle_{T}-v_{i, j-1 / 2}^{n+1}\langle F\rangle_{B}\right]
\end{aligned}
$$

where subscripts $\mathrm{B}, \mathrm{T}, \mathrm{L}$ and $\mathrm{R}$ denote quantities taken at the bottom, top, left and right sides of cell $(\mathrm{i}, \mathrm{j})$, and the superscript $\delta$ is a constant equal to 1 in cylindrical and 0 in Cartesian geometry. Bracketed quantities $\langle F\rangle$ are the fractional fluid volumes across each cell boundary. Special treatment of computing fractional fluid volume $\langle F\rangle$ must be taken to preserve the discontinuous nature of free surfaces. These quantities are estimated from the $\mathrm{H}-\mathrm{N}$ reconstruction algorithm, which is based on a type of donor-acceptor flux approximation [67]. The basic idea is to reconstruct a free surface either horizontally or vertically according to the value of $F$ which is downstream and upstream of a cell boundary, then to calculate the quantities of fractional fluid volume crossing this cell boundary. 
This method may be understood by considering the fractional volume of fluid that is fluxed through the unit cross sectional area of the right cell face during a time step $\Delta t$. The total flux of volume crossing the area is $u_{i+1 / 2, j}^{n+1} \Delta t$. The sign of $u_{i+1 / 2, j}^{n+1}$ determines the donor and acceptor cells with the donor cell losing fluid and the acceptor cell gaining fluid. The fractional volume of fluid crossing this boundary is the sum of an "upstream" value $F_{i a d, j}$ plus an increment $\delta F$ :

$$
\langle F\rangle_{R}=F_{i a d, j}+\delta F
$$

where the VOF increment can be written as:

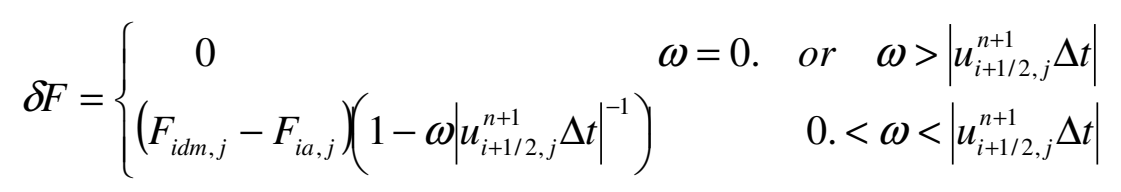

The relative value of a "void width" $\omega$ in the above equation is the distance from the flux boundary to the reconstructed free surface as shown in Figure 3.5(a) and (b). Hence, $\omega$ is nonzero only when the reconstructed free surface is perpendicular to the fluxing direction. The quantity of $\omega$ is determined by keeping the same amount of unoccupied volume of fluid before and after free surface reconstruction, i.e., $S_{1}$ equals $S_{2}$. Therefore, it has the expression:

$$
\omega=\left(\frac{F_{i d m, j}-F_{i d, j}}{F_{i d m, j}-F_{i a, j}}\right) \delta x_{i}
$$


The donor $(i d)$, acceptor (ia) and donor-minus (idm) cell indices are always $\mathrm{i}, \mathrm{i}+1$ and $\mathrm{i}-1$, respectively. The cell index iad refers to either the acceptor cell (ia) or the donor cell (id), depending upon the orientation of the interface relative to the direction of flow. The acceptor cell $(\mathrm{ia})$ is used when the free surface is mostly vertical to the direction of flow, as seen in the cases of Figure 3.4(a) and (b). Otherwise, the donor cell (id) is used for the case with the horizontal free surface reconstruction.
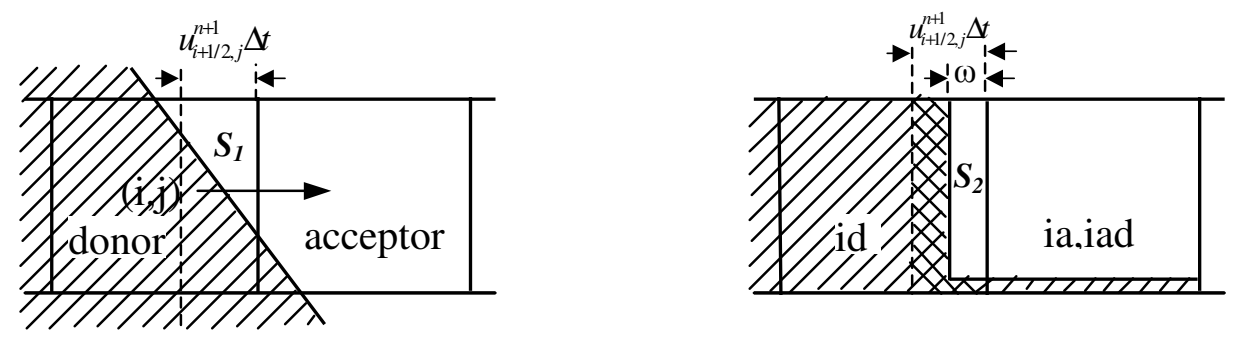

(a)
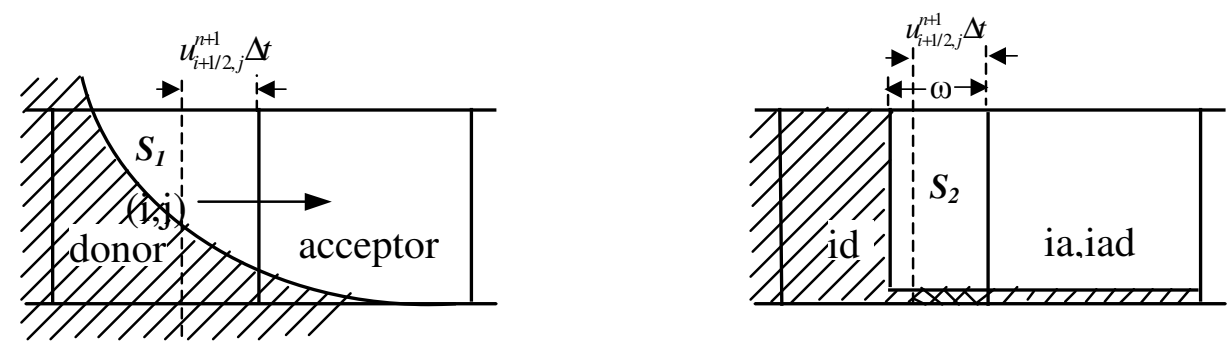

(b)
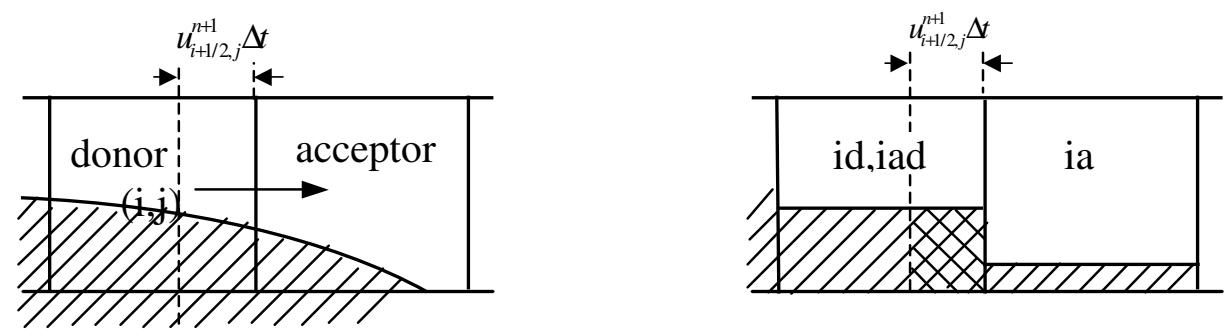

(c)

Figure 3.5 Examples of Free Surface Shapes and Reconstructions in the Advection of $F$ through the Cell Face 
Figure 3.5(a) and (b) show the cases of the reconstructed free surface vertical to the direction of flow. Figure 3.5(c) shows the example of reconstructed free surface horizontal to the direction of flow. The crosshatched regions shown in the figures are the actual volume of fluid fluxed, while the dashed lines indicate the total volume being advected. Similar calculations are performed for estimates of flux quantities $\langle F\rangle_{L},\langle F\rangle_{T}$ and $\langle F\rangle_{B}$.

\subsection{Modeling of Surface Tension}

Surface tension at a free surface is modeled with a localized volume force prescribed by the continuum surface force (CSF) model [62]. Instead of a surface tensile force or a surface pressure boundary condition applied at a discontinuity, a volume force acts on fluid lying within finite thickness transition regions continuously. Surface tension modeled with the continuum method eliminates the need for interface reconstruction, and can be easily calculated by applying an extra body force in the momentum equation (3.2).

In its standard form, the surface tension force per unit interfacial area is

$$
\vec{F}_{s a}\left(\vec{x}_{s}\right)=\sigma \kappa\left(\vec{x}_{s}\right) \hat{n}\left(\vec{x}_{s}\right)
$$

Where $\sigma, \kappa$, and $\hat{n}$ are the surface tension coefficient, the surface curvature and the unit normal to the surface at a point of $\vec{x}_{s}$ on a free surface. 
It can be reformulated to as a volume force by satisfying Green's Theory:

$$
\lim _{h \rightarrow 0} \int_{\Delta V} \vec{F}_{s v}(\vec{x}) d V=\int_{\Delta S} \vec{F}_{s a}\left(\vec{x}_{s}\right) d S
$$

The volume force $\vec{F}_{s v}$ is identified as :

$$
\vec{F}_{s v}(\vec{x})=\sigma \kappa(\vec{x}) \frac{\nabla \tilde{c}(\vec{x})}{[c]}
$$

$\tilde{c}(\vec{x})$ is the fluid color function, which varies smoothly over a thickness $h$ of transition regions at an interface, as shown in Figure 3.6. $[c]$ is the jump in color, $c_{2}-c_{1}$. For the case of incompressible flow, the color function $\tilde{c}(\vec{x})$ can be set as the VOF function $F(\vec{x})$ :

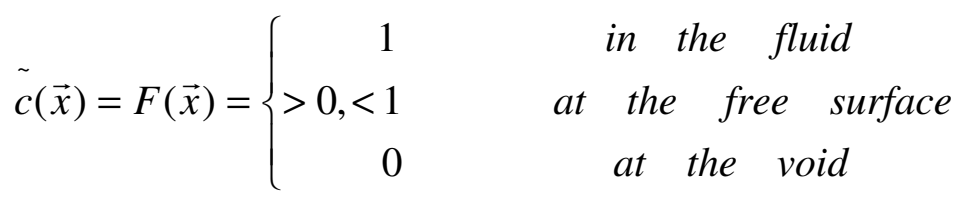

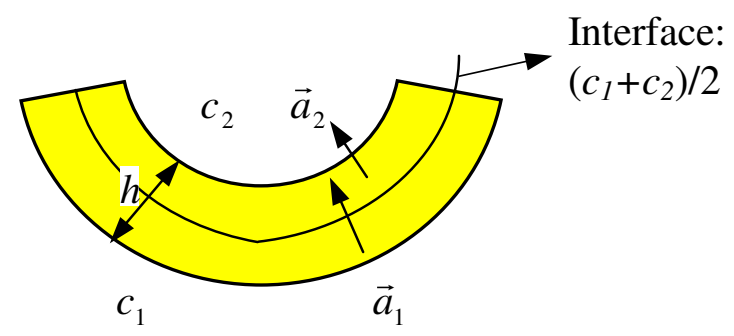

Figure 3.6 The transition zone with thickness $h$ at the interface

The volume force becomes

$$
\vec{F}_{s v}(\vec{x})=\sigma \kappa(\vec{x}) \nabla F(\vec{x})
$$

The free surface curvature $\kappa$ is calculated from

$$
\kappa=-(\nabla \cdot \widehat{n})
$$


where $\hat{n}$ is the unit normal to the surface,

$$
\widehat{n}=\frac{\vec{n}}{|\vec{n}|}
$$

Then, the curvature of free surface $\kappa$ is given by

$$
\kappa=\frac{1}{|\vec{n}|}\left[\left(\frac{\vec{n}}{|\vec{n}|} \cdot \nabla\right)|\vec{n}|-(\nabla \cdot \vec{n})\right]
$$

While normal $\vec{n}$ is a gradient of the color function

$$
\vec{n}=\nabla \tilde{c}(\vec{x})=\nabla F(\vec{x})
$$

If the above term $\vec{F}_{s v}(\vec{x})$ is added directly into the momentum equation as an extra body force, it causes that fluid accelerations, induced by surface tension, also depend on the value of local fluid density. Its application results in undesirable expansion or compression of the transition region at the free surface.

Hence, the volume force $\vec{F}_{s v}(\vec{x})$ is modified by multiplying an additional function

$$
g(x)=2 \tilde{c}(\vec{x}) /\left(c_{1}+c_{2}\right)=2 F(\vec{x})=2 \rho / \rho_{f}
$$

without changing the value of the integral in equation (3.52), since $g(x)=1$ as $h \rightarrow 0$. With this modification, the volume force becomes

$$
\vec{F}_{s v}(\vec{x})=\sigma \kappa(\vec{x}) \nabla F(\vec{x}) g(\vec{x})
$$

Adding $\vec{F}_{s v}(\vec{x})$ into the momentum equation (3.2) as an extra body force, yields

$$
\rho \frac{D \vec{v}}{D t}=-\nabla p+\nabla \cdot \vec{\tau}+\vec{F}_{b}+\vec{F}_{s v}(\vec{x})
$$


Through this modification, the fluid acceleration due to surface tension

$$
\vec{a}^{\prime}=\frac{\vec{F}_{s v}(\vec{x})}{\rho}=2 \sigma \kappa(\vec{x}) \nabla F(\vec{x}) / \rho_{f}
$$

depends only on the density gradient. Hence, better results are obtained because the application of the modified volume force avoids undesirable expansion and compression over the transition region at the free surface.

\subsection{Calculation of Electromagnetic Force}

The effect of welding current on the metal transfer includes the determination of the electromagnetic force, which is part of the body force in the momentum equation 3.2. According to Lorentz's law, the electromagnetic force generated by the welding current and self-induced magnetic field is expressed as:

$$
\vec{F}_{m}=\vec{J} \times \vec{B}
$$

where $\vec{J}$ is current density and $\vec{B}$ is the magnetic flux density. The magnetic flux density of the self-induced magnetic field $\vec{B}$ is derived from Ampere's law

$$
B_{\theta}=\frac{\mu_{0}}{x} \int_{0}^{x} J_{y} x d x
$$

and the current density $\vec{J}$ is calculated from Ohm's law

$$
J_{x}=-\sigma \frac{\partial \phi}{\partial x} \quad J_{y}=-\sigma \frac{\partial \phi}{\partial y}
$$

Therefore, the electromagnetic force is written as

$$
\vec{F}_{m}=-J_{y} B_{\theta} \vec{i}+J_{x} B_{\theta} \vec{j}
$$


Assuming the electric field is quasi-steady-state and the electrical conductivity $\sigma$ is constant, the electric potential $\phi$, being the only unknown variable, can be calculated by solving the current continuity equation

$$
\frac{1}{r^{\delta}} \frac{\partial}{\partial x}\left(r^{\delta} \frac{\partial \phi}{\partial x}\right)+\frac{\partial^{2} \phi}{\partial y^{2}}=0
$$

The superscript $\delta$ is a constant equal to 1 in cylindrical and 0 in Cartesian geometry.

A finite difference form of the above current continuity equation (3.68) over the computational cell $(i, j)$ shown in Figure 3.7 can be written as follows:

$$
A_{\phi_{i, j}} \phi_{i, j}+B_{\phi_{i, j}} \phi_{i+1, j}+C_{\phi_{i, j}} \phi_{i, j+1}+D_{\phi_{i, j}} \phi_{i-1, j}+E_{\phi_{i, j}} \phi_{i, j-1}=S_{\phi_{i, j}}
$$

Where

$$
\begin{array}{ll}
A_{i, j}=\left(r^{\delta} \alpha\right)_{i+1 / 2, j}+\left(r^{\delta} \alpha\right)_{i-1 / 2, j}+\left(r^{\delta} \gamma\right)_{i, j+1 / 2}+\left(r^{\delta} \gamma\right)_{i, j-1 / 2} \\
B_{\phi_{i, j}}=-\left(r^{\delta} \alpha\right)_{i+1 / 2, j}, & C_{\phi_{i, j}}=-\left(r^{\delta} \gamma\right)_{i, j+1 / 2} \\
D_{\phi_{i, j}}=-\left(r^{\delta} \alpha\right)_{i-1 / 2, j}, & E_{\phi_{i, j}}=-\left(r^{\delta} \gamma\right)_{i, j-1 / 2} \\
S_{\phi_{i, j}}=0 &
\end{array}
$$

and $\alpha, \gamma$ are defined by

$$
\alpha_{i, j}=\frac{y_{j}-y_{j-1}}{x_{i}-x_{i-1}}, \quad \gamma_{i, j}=\frac{x_{i}-x_{i-1}}{y_{j}-y_{j-1}}
$$

The above finite difference equations can be solved by solving a matrix equation:

$$
\mathbf{M}_{\phi} \phi=\mathbf{S}_{\phi}
$$

with integration of boundary conditions. One of the important boundary conditions here is the current density distribution on the droplet surface. 


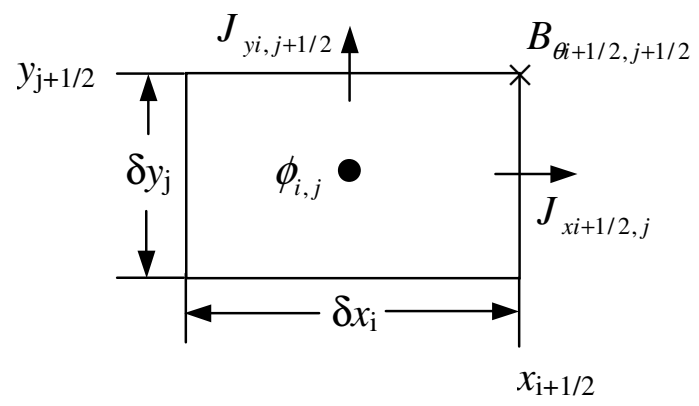

Figure 3.7 Layout of electromagnetic variables in a computational cell centered at $(\mathrm{i}, \mathrm{j})$

In the present study, a Gaussian current density distribution has been proposed and adopted. The current density for the free surface cell $(i, j)$ is written as:

$$
\begin{aligned}
& J_{s i, j}=I \cdot f(i, j) / \sum_{n}\left(S_{i, j} \cdot f(i, j)\right) \\
& f(i, j)=\frac{1}{\sqrt{2 \pi}} \exp \left(-\xi_{i, j}^{2} / 2\right) \\
& \xi_{i, j}=\frac{X_{i, j}}{D}
\end{aligned}
$$

where $X_{i, j}$ is the arc length on the droplet surface between the lowest point on the droplet and the free surface cell $(i, j)$ and $\mathrm{n}$ is the number of the free surface cells on the droplet. The current density relates to the gradient of electric potential:

$$
\vec{J}_{s i, j}=-\sigma \frac{\partial \phi}{\partial \vec{n}_{s}}
$$

Hence, the gradient of electric potential on the free surface of the droplet as a critical boundary condition must be integrated to solve the current continuity equation (3.69). However, the application of this Neumann boundary condition is complicated by the 
enforcement on an internal free surface. Special treatment to apply this boundary condition on the free surface cell has been performed in the present study.

For a free surface cell $(i+1, j)$, which contains a reconstructed vertical free surface and has fluid on the left hand side (Figure 3.8), the boundary condition can be written as:

$$
\begin{gathered}
\frac{\phi_{i+1, j}-\phi_{i, j}}{0.5\left(\Delta x_{i}+\Delta x_{i+1}\right)}=-\frac{J_{s i, j}}{\sigma} \\
\phi_{i+1, j}=\phi_{i, j}-0.5\left(\Delta x_{i}+\Delta x_{i+1}\right) J_{s i, j} / \sigma
\end{gathered}
$$

Substituting $\phi_{i+1, j}$ into the current continuity equation (3.69) for the computational cell centered at $(i, j)$, the equation becomes

$$
\begin{gathered}
A_{\phi_{i, j}} \phi_{i, j}+B_{\phi_{i, j}}\left[\phi_{i, j}-0.5\left(\Delta x_{i}+\Delta x_{i+1}\right) J_{s i, j} / \sigma\right] \\
+C_{\phi_{i, j}} \phi_{i, j+1}+D_{\phi_{i, j}} \phi_{i-1, j}+E_{\phi_{i, j}} \phi_{i, j-1}=0 \\
\left(A_{\phi_{i, j}}+B_{\phi_{i, j}}\right) \phi_{i, j}+C_{\phi_{i, j}} \phi_{i, j+1}+D_{\phi_{i, j}} \phi_{i-1, j}+E_{\phi_{i, j}} \phi_{i, j-1} \\
=0.5 B_{\phi_{i, j}}\left(\Delta x_{i}+\Delta x_{i+1}\right) J_{s i, j} / \sigma
\end{gathered}
$$

Hence, the boundary condition is incorporated by resetting the coefficient of $\phi_{i, j}$ and $\phi_{i+1, j}$ in the current continuity equation for cell centered at $(i, j)$ to the following:

$$
\begin{aligned}
& A_{\phi_{i, j}}{ }^{\prime}=A_{\phi_{i, j}}+B_{\phi_{i, j}} \\
& B_{\phi_{i, j}}{ }^{\prime}=0
\end{aligned}
$$

and adding an extra source term:

$$
S_{\phi_{i, j}}{ }^{\prime}=0.5 B_{\phi_{i, j}}\left(\Delta x_{i}+\Delta x_{i+1}\right) J_{s i, j} / \sigma
$$

For the free surface cell $(i+1, j)$, the boundary condition equation (3.80) can also be enforced by setting the coefficients in current continuity equation as follows: 


$$
\begin{aligned}
& D_{\phi_{i+1, j}}=-A_{\phi_{i+1, j}} \\
& B_{\phi_{i+1, j}}=C_{\phi_{i+1, j}}{ }^{\prime}=E_{\phi_{i+1, j}}=0
\end{aligned}
$$

and

$$
S_{\phi_{i+1, j}}=-0.5 A_{\phi_{i+1, j}}\left(\Delta x_{i}+\Delta x_{i+1}\right) J_{s i, j} / \sigma
$$

Similar treatments are performed for free surface cells, which have fluid on the right hand side, on the top, and on the bottom.

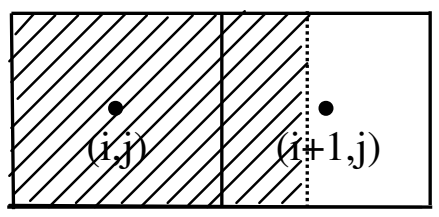

(a)

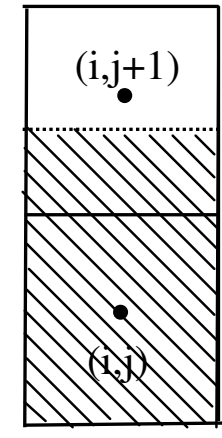

(c)

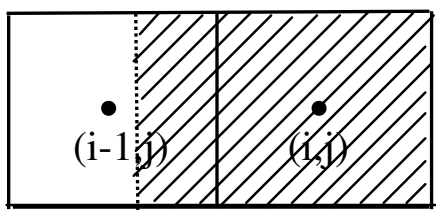

(b)

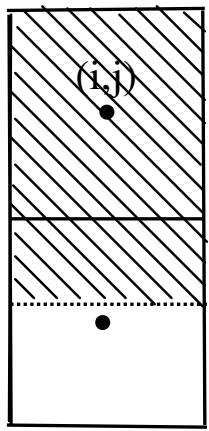

(d)

Figure 3.8 Examples of free surface cells using for boundary condition application
(a) Fluid on the left hand side
(b) Fluid on the right hand side
(c) Fluid on the bottom
(d) Fluid on the top 
For the free surface cell $(i-1, j)$, which contains a reconstructed vertical free surface and has fluid on the right hand side, the Neumann boundary condition is incorporated by resetting the coefficients and adding source terms in the current continuity equation for computational cells centered at $(i, j)$ and $(i-1, j)$ :

$$
\begin{aligned}
& A_{\phi_{i, j}}{ }^{\prime}=A_{\phi_{i, j}}+D_{\phi_{i, j}} \\
& D_{\phi_{i, j}}{ }^{\prime}=0 \\
& S_{\phi_{i, j}}{ }^{\prime}=0.5 D_{\phi i, j}\left(\Delta x_{i}+\Delta x_{i-1}\right) J_{s i, j} / \sigma
\end{aligned}
$$

and

$$
\begin{aligned}
& B_{\phi_{i-1, j}}{ }^{\prime}=-A_{\phi_{i-1, j}} \\
& C_{\phi_{i-1, j}}=D_{\phi_{i-1, j}}=E_{\phi_{i-1, j}}=0 \\
& S_{\phi_{i-1, j}}=-0.5 A_{\phi_{i-1, . j}}\left(\Delta x_{i}+\Delta x_{i-1}\right) J_{s i, j} / \sigma
\end{aligned}
$$

For the free surface cell $(i, j+1)$, which contains a reconstructed horizontal free surface and has fluid on the bottom, the Neumann boundary condition is incorporated by resetting the coefficients and adding source terms in the current continuity equation for computational cells centered at $(i, j)$ and $(i, j+1)$ :

$$
\begin{aligned}
& A_{\phi_{i, j}}{ }^{\prime}=A_{\phi_{i, j}}+C_{\phi_{i, j}} \\
& C_{\phi_{i, j}}{ }^{\prime}=0 \\
& S_{\phi_{i, j}}{ }^{\prime}=0.5 C_{\phi_{i, j}}\left(\Delta y_{j}+\Delta y_{j+1}\right) J_{s i, j} / \sigma
\end{aligned}
$$

and

$$
E_{\phi_{i, j+1}}{ }^{\prime}=-A_{\phi_{i, j+1}}
$$




$$
\begin{aligned}
& B_{\phi_{i, j+1}}{ }^{\prime}=C_{\phi_{i, j+1}}{ }^{\prime}=D_{\phi_{i, j+1}}{ }^{\prime}=0 \\
& S_{\phi_{i, j+1}}{ }^{\prime}=-0.5 A_{\phi_{i, j+1}}\left(\Delta y_{j}+\Delta y_{j+1}\right) J_{s i, j} / \sigma
\end{aligned}
$$

For the free surface cell $(i, j-1)$, which contains a reconstructed horizontal free surface and has fluid on the top, the Neumann boundary condition is incorporated by resetting the coefficients and adding source terms in the current continuity equation for computational cells centered at $(i, j)$ and $(i, j-1)$ :

$$
\begin{aligned}
& A_{\phi_{i, j}}{ }^{\prime}=A_{\phi_{i, j}}+E_{\phi_{i, j}} \\
& E_{\phi_{i, j}{ }^{\prime}}=0 \\
& S_{\phi_{i, j}{ }^{\prime}}=0.5 E_{\phi_{i, j}}\left(\Delta y_{j}+\Delta y_{j-1}\right) J_{s i, j} / \sigma
\end{aligned}
$$

and

$$
\begin{aligned}
& C_{\phi_{i, j-1}}=-A_{\phi_{i, j-1}} \\
& B_{\phi_{i, j-1}}=D_{\phi_{i, j-1}}=E_{\phi_{i, j-1}}=0 \\
& S_{\phi_{i, j-1}}=-0.5 A_{\phi_{i, j-1}}\left(\Delta y_{j}+\Delta y_{j-1}\right) J_{s i, j} / \sigma
\end{aligned}
$$

The finite difference form of the current continuity equation (3.69) and the enforced boundary condition (3.80) results in second order accuracy.

After solving the current continuity equation (3.75) to get electric potential $\phi$ centered at the computational cell, the current density at the face of cell $(i, j)$ can be calculated by equation (3.66):

$$
J_{x i+1 / 2, j}=-\sigma \frac{\phi_{i+1, j}-\phi_{i, j}}{0.5\left(\Delta x_{i}+\Delta x_{i+1}\right)}
$$




$$
J_{y_{i, j+1 / 2}}=-\sigma \frac{\phi_{i, j+1}-\phi_{i, j}}{0.5\left(\Delta y_{j}+\Delta y_{j+1}\right)}
$$

and the electromagnetic flux density can be calaculated by equation (3.65)

$$
B_{\theta i+1 / 2, j+1 / 2}=\frac{\mu_{0}}{x_{i}} \sum_{0}^{i} J_{y_{i, j+1 / 2}} x_{i} \Delta x_{i}
$$

Electromagnetic force, which directly influences fluid velocity as a body force, is evaluated at the right face of the computational cell for the x-component and at the left face of the computational cell for the y-component. From equation (3.67), the $x$ component of electromagnetic force at $(i+1 / 2, j)$ becomes

$$
\begin{aligned}
& \vec{F}_{m x i+1 / 2, j}=-J_{y i+1 / 2, j} B_{\theta_{i+1 / 2, j}} \\
& =-\left(\frac{1}{2}\left(\left(J_{y i, j+1 / 2}+J_{y i, j-1 / 2}\right) \Delta x_{i+1}+\left(J_{y i+1, j+1 / 2}+J_{y i+1, j-1 / 2}\right) \Delta x_{i}\right) /\left(\Delta x_{i}+\Delta x_{i+1}\right)\right) \\
& \quad \times\left(\frac{1}{2}\left(B_{\theta i+1 / 2, j+1 / 2}+B_{\theta i+1 / 2, j-1 / 2}\right)\right)
\end{aligned}
$$

and the $y$-component of electromagnetic force at $(i, j+1 / 2)$ becomes

$$
\begin{aligned}
\vec{F}_{m y_{i, j+1 / 2}}=-J_{x i, j+1 / 2} B_{\theta_{i, j+1 / 2}} \\
=-\left(\frac{1}{2}\left(\left(J_{x i+1 / 2, j}+J_{x i-1 / 2, j}\right) \Delta y_{j+1}+\left(J_{x i+1 / 2, j+1}+J_{x i-1 / 2, j+1}\right) \Delta y_{j}\right) /\left(\Delta y_{j}+\Delta y_{j+1}\right)\right) \\
\quad \times\left(\frac{1}{2}\left(B_{\theta i+1 / 2, j+1 / 2}+B_{\theta i-1 / 2, j+1 / 2}\right)\right)
\end{aligned}
$$

Copyright $\odot$ Ge Wang 2007 


\section{Chapter 4}

\section{Metal Transfer in Constant Current GMAW}

Based on the physical model and numerical schemes discussed in previous chapters, the dynamic characteristics of the metal transfer process in gas metal arc welding are simulated and analyzed. The numerical calculation is first carried out for the metal transfer process under constant current and comparisons are made between calculated results and experimental data. It is found that the current density distribution on the droplet surface has a significant effect on the simulation results. The results calculated assuming a gaussian current distribution on the droplet surface are in good agreement with the experimental data. The droplet profiles, electric potential, and velocity distributions within the droplet are calculated and presented. The analysis is conducted to find the most dominant effects on the different metal transfer modes. The transition of metal transfer mode has been considered as a critical phenomenon, which changes dramatically over a narrow range of welding current. This transition zone has been investigated numerically which shows irregularity in detached droplet sizes like experimental observation. The mechanism of the unique metal transfer behavior during the transition is discussed based on the numerical calculation. 


\subsection{Introduction}

In constant current GMAW, metal transfer can take place in two principle modes: globular transfer and spray transfer. Globular transfer occurs in a low current welding process. In globular transfer, the diameter of a detached droplet is much greater than that of the electrode. As welding current increases, spray transfer with detached droplet size about the same or smaller than that of the electrode takes place. Spray transfer can be further classified as drop (projected) spray or streaming spray, depending on the diameter of the detached droplet in relation to that of the electrode: approximately the same in drop spray or much smaller in streaming spray. It is found experimentally that there is a sharp transition in the droplet detachment frequency and size when the metal transfer occurs within the narrow current range between the globular and spray transfer modes. A bifurcation in the droplet detachment frequency and the droplet size has been observed in the middle of the transition current range [8-10]. The numerical results presented in the previous literature all failed to catch this transition current range accurately and did not disclose the distinct behavior of the metal transfer process under the transition current.

The two theories, most used for prediction of metal transfer in early theoretical studies, are the static force balance theory (SFBT) [25-27] and the pinch instability theory (PIT) [28-30]. In 1993, Kim and Eagar [68] compared results provided by those two theories with experimental measurements. They show that neither theory predicts results consistent with the experimental data, especially in the transition region from globular to spray transfer. Nemchinsky [32] developed a steady state equation to describe the 
equilibrium shape of a pendant droplet. A threshold current is calculated to describe an important feature of metal transfer - the transition current from globular to spray transfer. However, the results fail to give the range of transition current and the unique behavior of the metal transfer process under the transition. More recently, advanced CFD techniques have been introduced to study the metal transfer phenomena and have made significant contributions to this area.

The volume of fluid (VOF) method has proved to be an effective means to solve fluid flow with free surfaces in CFD and has been adopted in some recent numerical studies of gas metal arc welding. Choi, Yoo and Kim [43] analyzed the dynamic characteristics of the metal transfer processes by using the VOF method. They found that the current density on the droplet surface, which determines the distributions of the voltage and current density within the droplet, has significant effects on the detached droplet profile and size. Their results, given under the assumption of uniform or linear current density distribution over the droplet surface, still show discrepancies from the experimental measurements and especially in the current range of interest - the transition region from globular to spray transfer. Fan and Kovacevic [42] used the assumption of uniform distributed axial current density over any horizontal cross section of the droplet: $j_{z}=\frac{I(z)}{2 \pi R_{d}^{2}}$ with a linear approximation of current flowing through the droplet at distance of $\mathrm{z}$ from its tip: $I(z)=I \cdot z / H$ to calculate globular transfer in GMAW. Their results show good agreement with the experimental results only under globular transfer, for which the electromagnetic force has less influence. In this thesis, a transient twodimensional model is developed on the base of RIPPLE that uses a volume of fluid 
(VOF) method to capture the free surface and a continuum surface force (CSF) model to calculate surface tension. The electromagnetic force due to the welding current is estimated by assuming a Gaussian type of current density distribution over the droplet surface.

\subsection{Results and Discussions}

In this dissertation, the calculated results are presented for the physical cases based on the work of Kim and Eagar [68]. Simulations are carried out for a mild steel electrode with $1.6 \mathrm{~mm}$ diameter and a wire feed rate of $70 \mathrm{~mm} / \mathrm{s}$. The welding current varies from 150 to $320 \mathrm{~A}$, which covers the range of globular and spray metal transfer. The material properties taken from the work of Chio, Yoo and Kim [43] are listed in Table 1. The dimension of the solution domain is $4 \mathrm{~mm}$ and $14 \mathrm{~mm}$ in the $\mathrm{x}$ and $\mathrm{y}$ directions respectively. A uniform computational mesh with mesh spacing of $0.1 \mathrm{~mm}$ in each coordinate direction is used. For grid consideration, the mesh spacing was varied between $0.08 \mathrm{~mm}$ to $0.16 \mathrm{~mm}$. it was found that the predicted average droplet sizes remain unchanged. Although the change of mesh spacing leads to a change on the details of predicted results in the transition current range (between 230 to $260 \mathrm{~A}$ ), the averaged droplet sizes were found to be the same. The time step is adjusted automatically according to linear stability constraints during the course of a calculation. Calculations were executed first on a SGI-Origin-2100 workstation and then on a HP SDX superdome supercomputer. Since the HP SDX superdome supercomputer allow reducing significantly the CPU times in comparison with the SGI-Origin -2100 workstation, the 
CPU times given out in this paper are based on the calculations performed on the HP supercomputer.

\subsubsection{Effects of Surface Current Density Distribution}

Calculations were performed and results were compared for three different assumptions of current density distribution over droplet surface as mentioned previously in Chapter 2. The detached-droplet sizes predicted using a Gaussian current density distribution over the droplet surface for a range of welding currents are shown in Figure 4.1. The diameter of the detached droplet is calculated by averaging the total molten metal input over the number of the detached droplets during a period of welding time. They are compared with experimentally measured data [68] as well as the results calculated by assuming constant and linear current density distributions over the droplet surface [43].

As seen in Figure 4.1, the average detached droplet size decreases with increasing welding current. There exists a transition current range from 230 to $260 \mathrm{~A}$, over which a significant change of the detached droplet size occurs and the metal transfer mode changes from globular to spray. The images captured in a recent study using a high-speed video system also showed irregularity in detached droplet sizes occurring in the transition from globular to spray mode [10]. The results predicted using a Gaussian current density distribution show better agreement with the experimental data than the results calculated by using a constant or linear current density distribution on the droplet surface. In 
particular, the transition current range and the behavior of droplet development in this zone are predicted accurately by using a Gaussian current density distribution assumption.

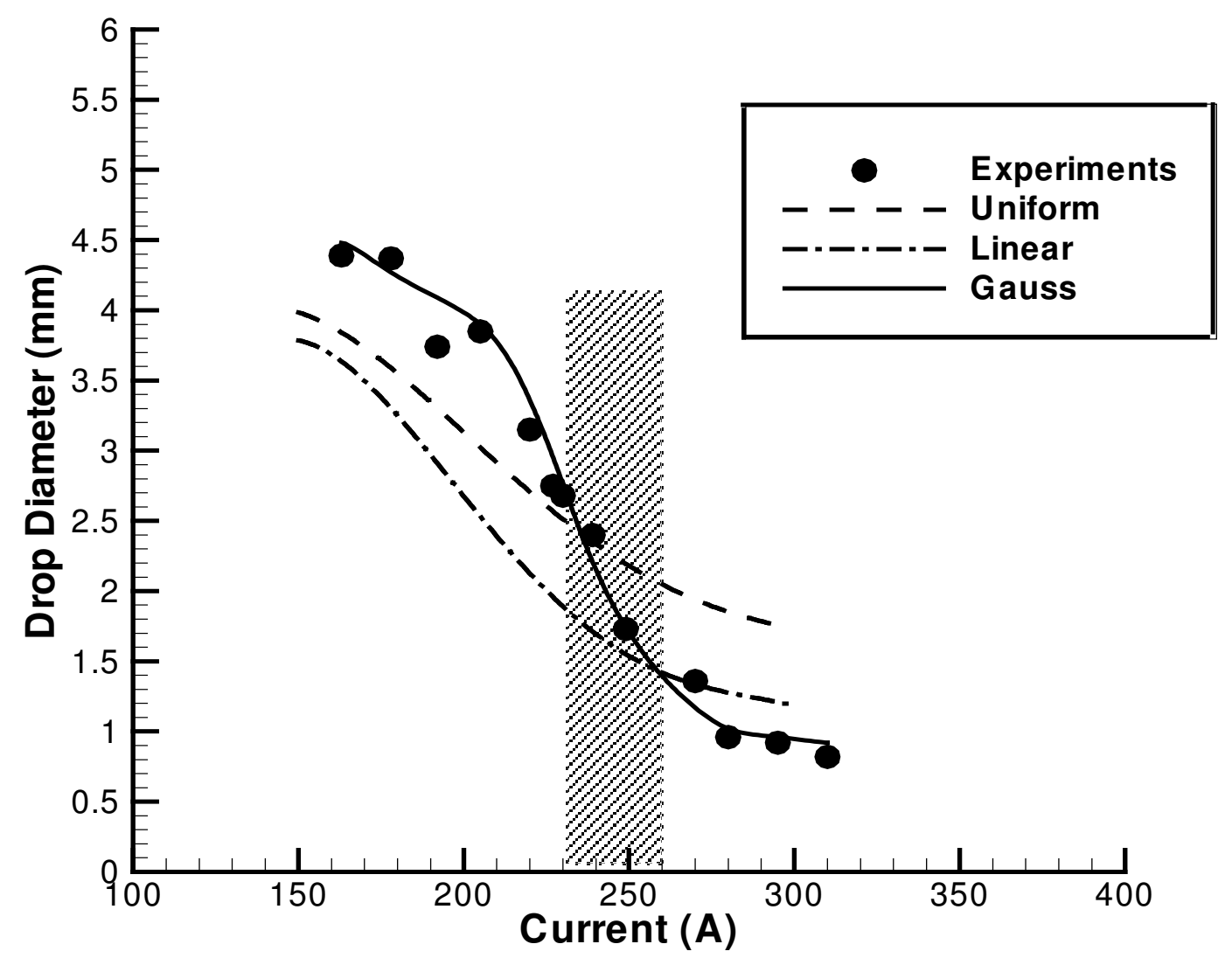

Figure 4.1 Comparison of predicted average droplet sizes under different current density distribution with experimental results 


\subsubsection{Dominant Effects for Different Metal Transfer Mode}

Figures 4.2, 4.3 and 4.4 show the droplet profiles, electric potential, and velocity distributions within the droplet during metal transfer processes, which were calculated under the assumption of a Gaussian current density distribution on the droplet surface, for welding currents of $160 \mathrm{~A}, 300 \mathrm{~A}$ and $250 \mathrm{~A}$ respectively. These three cases represent globular transfer with large droplets detached under the low welding current, spray transfer with small droplets detached under the higher welding current, and the transition from globular to spray transfer with non-uniform droplets within a narrow transition current range. The dominant effects affecting the dynamic development of the molten droplet and thereby resulting in a different mode of metal transfer are analyzed based on the numerical calculation.

Figure 4.2(a) shows the instantaneous profiles for droplet development at a current of 160A. The droplet grows at the tip of the electrode with a classic pendant drop shape, due to the balance between gravity and surface tension in the presence of decreasing electromagnetic force. Droplet detachment occurs after the neck shrinks. A droplet is detached about every $320 \mathrm{~ms}$ apart. The diameter of a detached droplet is about $4.4 \mathrm{~mm}$ with only slight variation. It took 6 hours of CPU time to simulate about 1.6 seconds of the real time process. 

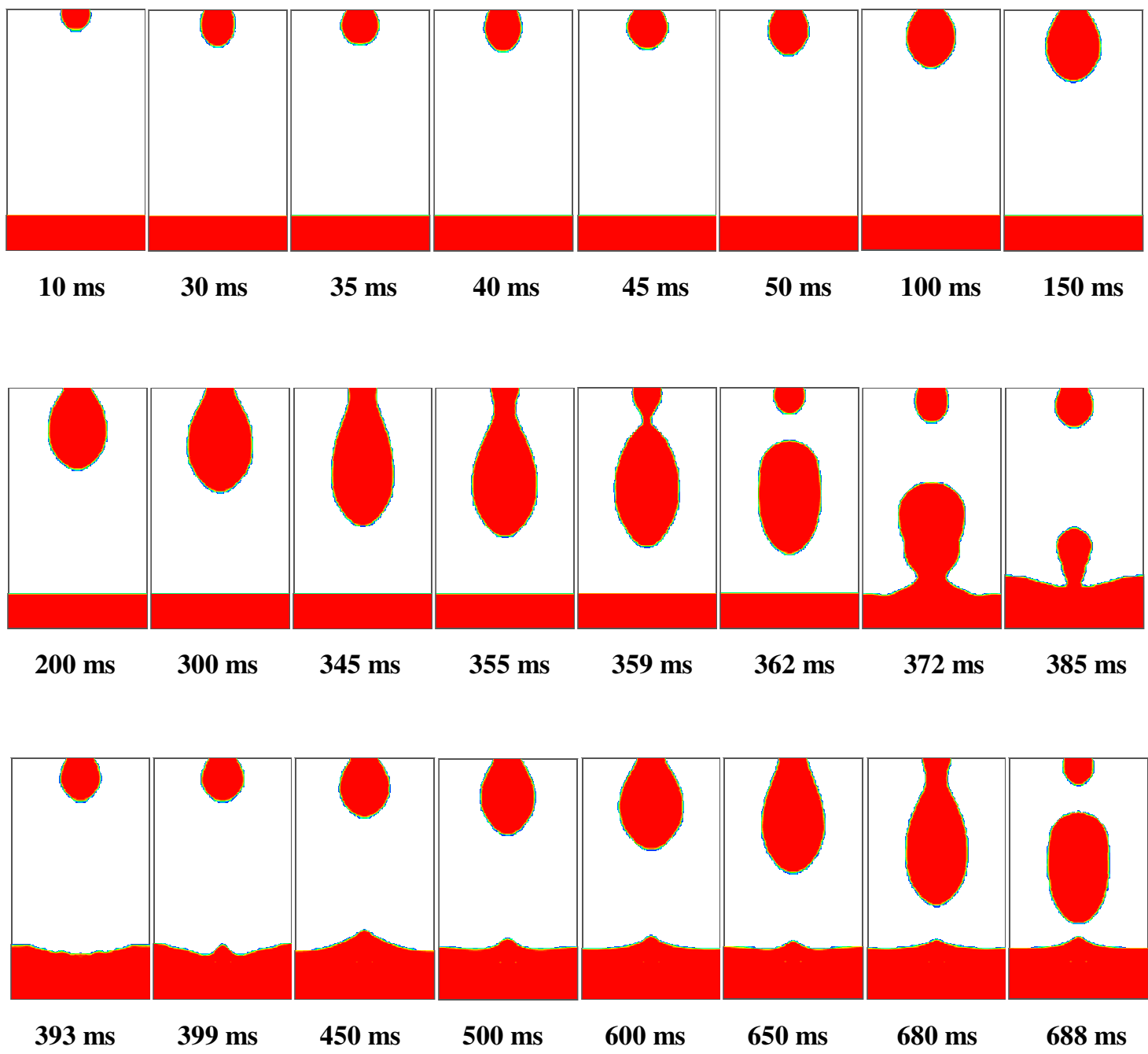

4.2(a) 

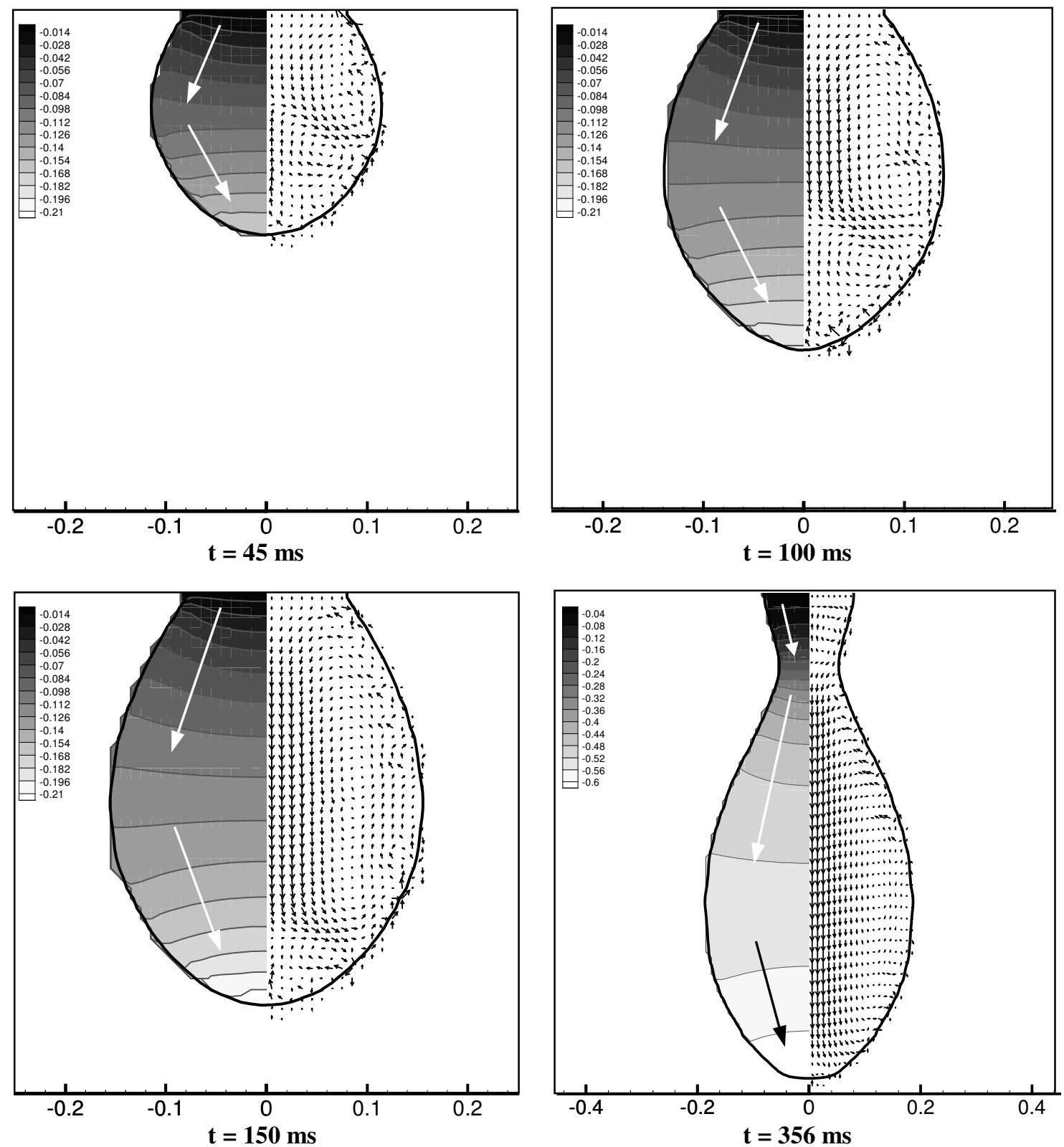

4.2(b)

Figure 4.2 Metal transfer process at a current of $160 \mathrm{~A}$

(a) Drop profiles (b) Electric potential and velocity distributions within the droplet 
Figure 4.2(b) shows the details of calculated electric potential and velocity distributions within the droplet for selected instants of time at a current of $160 \mathrm{~A}$. The current density and electromagnetic force are determined by the electric potential distribution. The current direction (marked in the figure) is normal to the curve of electric potential contour and points to the low potential. The magnitude of current density calculated from the gradient of the potential depends on the distance between contours of electric potential with same value. Different regions with concave contours and convex contours for the potential distribution are identified. The current diverges and the electromagnetic force with a downward component rises in the region with concave contours of electric potential distribution. The current converges and the electromagnetic force with an upward component rises in the region with convex contours of electric potential distribution.

There are two distinguishing regions with concave electric potential contours upside and convex potential contours downside observed before the neck forms in Figure 4.2(b). At $\mathrm{t}=45 \mathrm{~ms}$, the electromagnetic force dominates the droplet behavior compared to the gravitational force. The flow upside within the droplet is driven downward by the electromagnetic force, which has a downward component, and collides with the downside flow driven up by the electromagnetic force with an upward component. There is a counterclockwise vortex and a clockwise vortex formed. The competition between the two streams causes the observed vibration of the droplet. The magnitude of the current density decreases with the increase of pendant drop size, according to the observation of the enlargement in distance between potential contours with same value. Hence, the 
gravitational force dominates the flow instead of the electromagnetic force as the droplet becomes larger. The upside flow driven downward by gravitational and electromagnetic forces overcomes the downside flow driven upward by the electromagnetic force. Eventually, the electromagnetic force with an upward component can no longer compete with the gravitational force and the flow is driven down to hit the bottom of the droplet. There is another region with convex electric potential contour developed above the neck after it forms in the droplet. The flow driven up by the electromagnetic force with the upward component forms a clockwise vortex above the neck. From the distribution of the electric potential, the electromagnetic force accelerates the droplet detachment after the neck shrinks not only as an inward pinch force but also as a detaching force.

Figure 4.3(a) shows the instantaneous profiles for droplet development at a current of 300A. The electromagnetic force dominates the droplet detachment process compared to the gravitational force. The droplets become much smaller and the droplet detachment frequency is much greater. A droplet is detached about every $3.3 \mathrm{~ms}$ with a droplet diameter of $0.96 \mathrm{~mm}$. It took 5 hours of CPU time to simulate about 0.2 seconds of the real time process.

Figure 4.3(b) shows the details of the distributions of the calculated electric potential and velocity within the droplet for two selected instants of time at a current of 300A. There are two distinct regions with convex contour up and concave contour down in the observed electric potential distribution. A clockwise vortex and a counterclockwise vortex are induced by the electromagnetic force with an upward component and a 
downward component, respectively. The current density is very high within the droplet compared to the 160 A case. The electromagnetic force dominates the behavior of flow compared to the gravitational force. The surface tension cannot compete with the
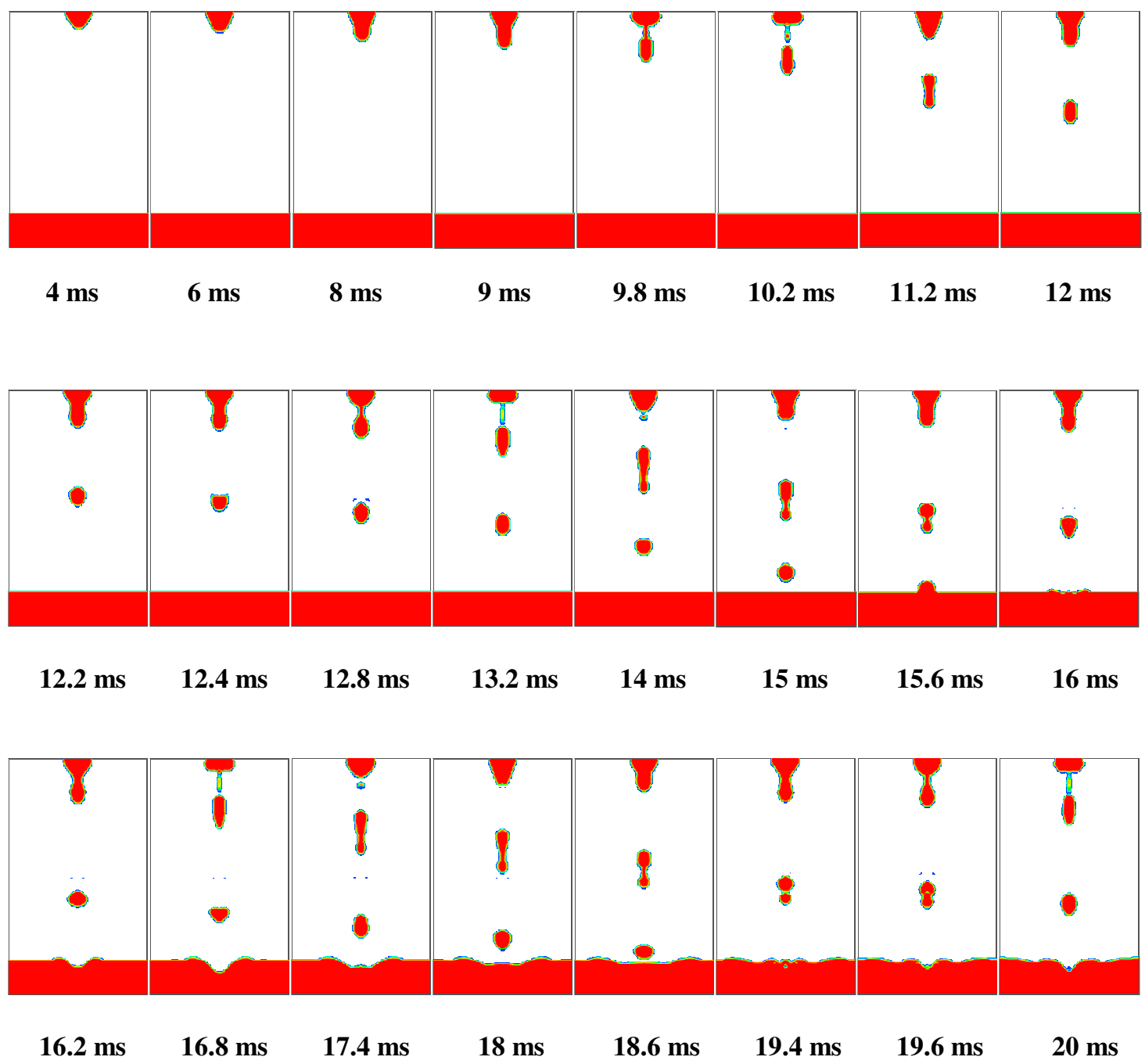

$4.3(\mathrm{a})$ 

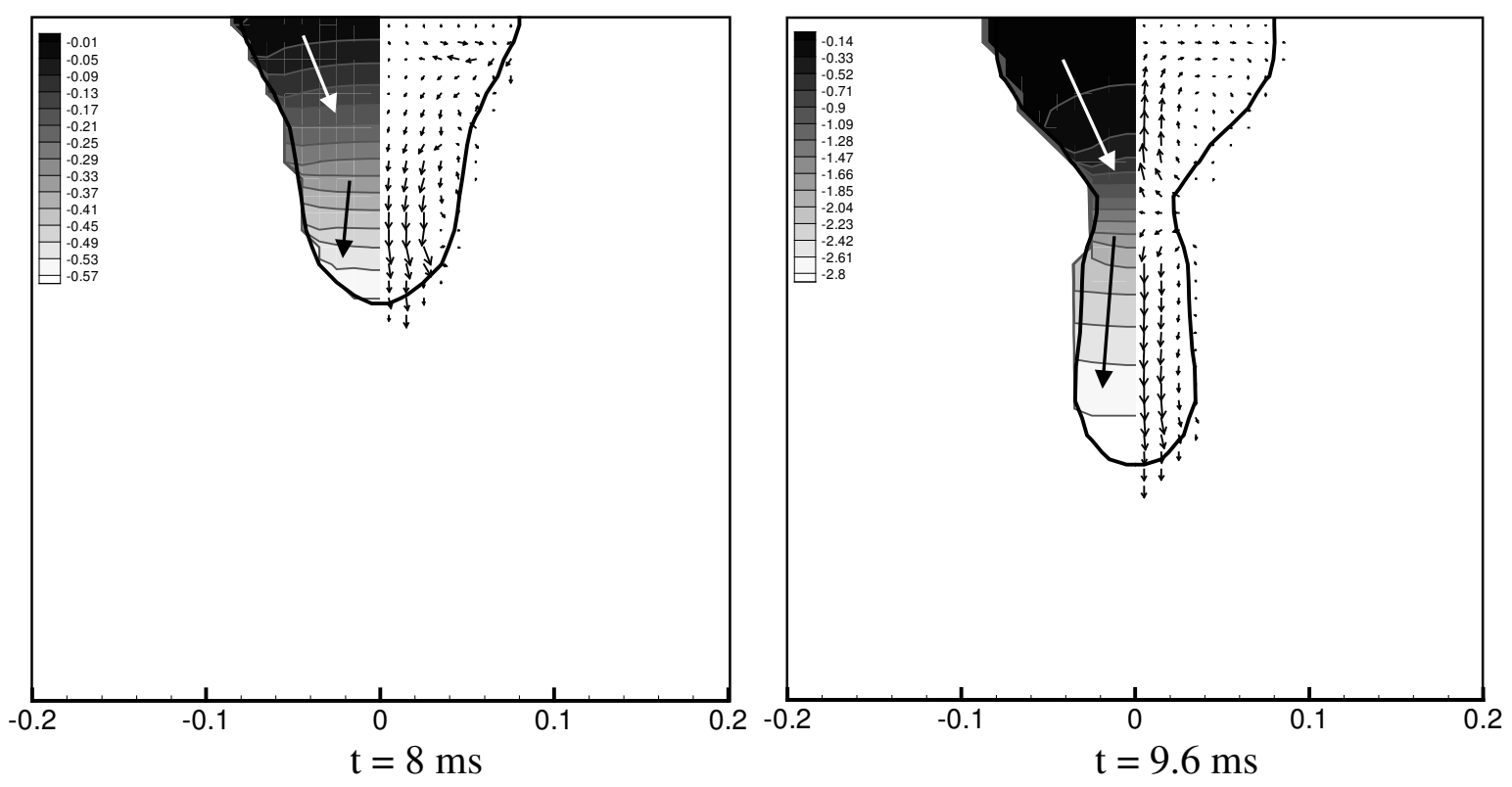

4.3(b)

Figure 4.3 Metal transfer process at a current of 300A

(a) Drop profiles (b) Electric potential and velocity distributions within the droplet

electromagnetic force before the droplet grows larger. The small droplet is detached by the electromagnetic force pinching inward and pulling apart.

Figure 4.4(a) shows the instantaneous profiles for droplet development at a current of 250A. The gravitational force and electromagnetic force both affect the droplet detachment process in the middle of the transition from globular to spray mode. The electromagnetic force helps generate a series of small droplets. However, the electromagnetic force is not large enough to detach the whole droplet and excess fluid accumulates on the tip of electrode. The electromagnetic force becomes weaker as this 


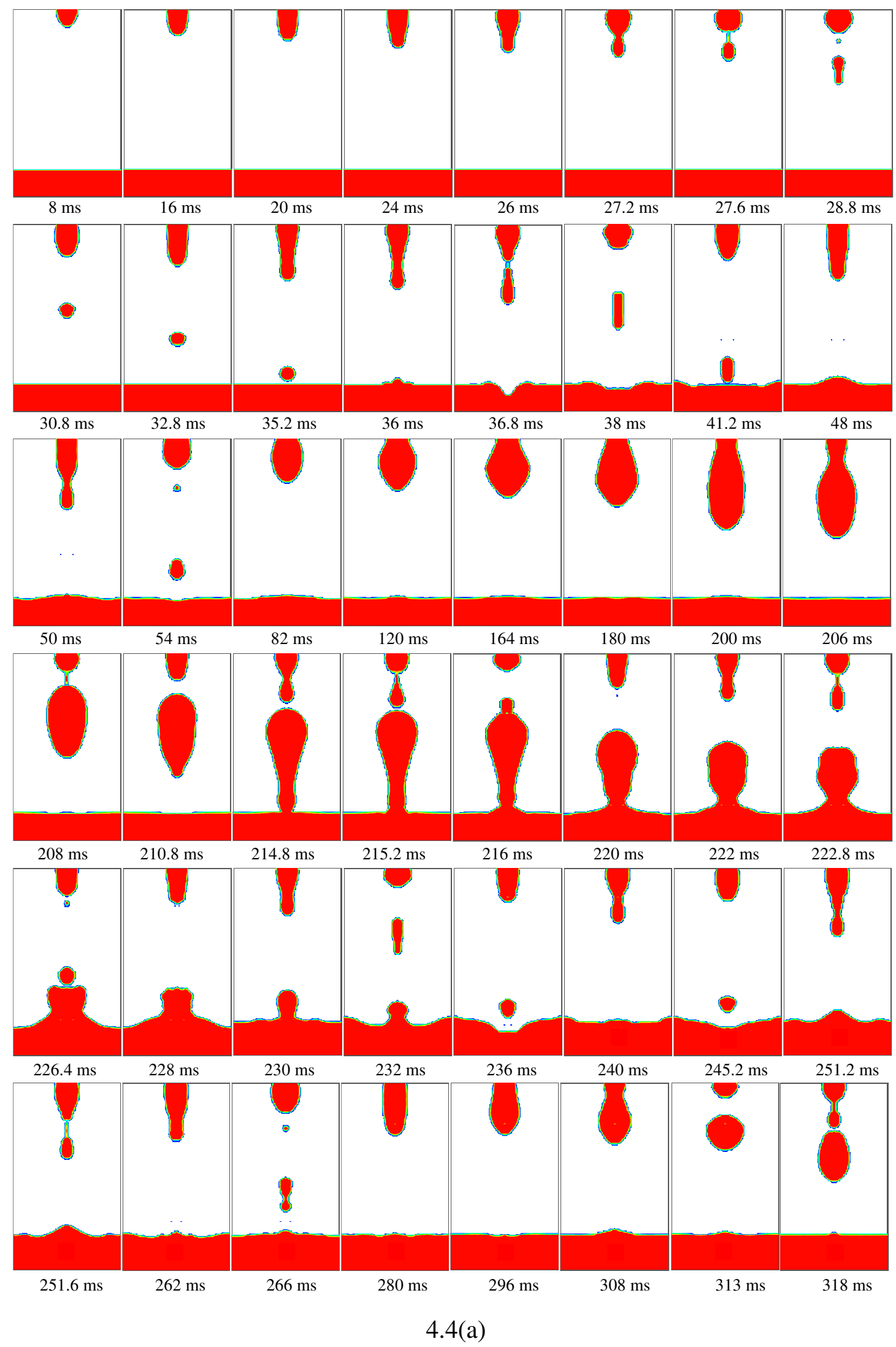



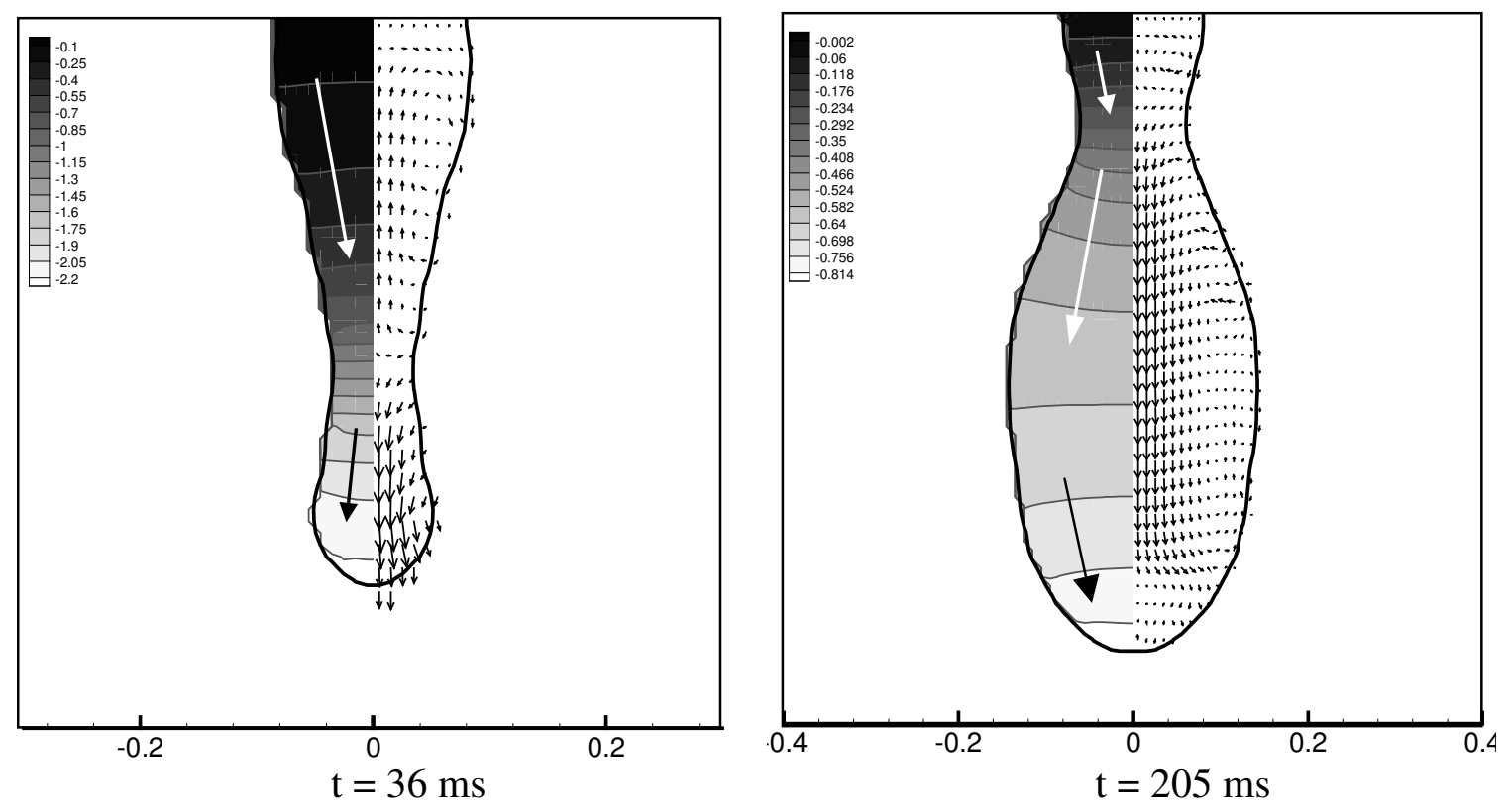

4.4(b)

Figure 4.4 Metal transfer process at a current of 250A

(a) Drop profiles (b) Electric potential and velocity distributions within the droplet

excess fluid grows. When enough fluid accumulates at the tip of the electrode, the electromagnetic force will not be able to detach the droplet fluid. A large droplet forms and is finally detached by the gravitational force. Hence, a bifurcation in the drop detachment frequency and the droplet size is captured by the calculation. A large droplet is formed and detached between every few small droplets. The phenomena are consistent with the experimental observation in pervious studies [10]. It took 8 hours of CPU time to simulate about 1 second of the real time process. 
Figure 4.4(b) shows the details of the instantaneous electric potential and velocity distributions within the droplet for a current of $250 \mathrm{~A}$, which lies in the middle of the transition from globular to spray mode. A small droplet forms on the tip of electrode with electric potential and velocity distributions similar to the case representing spray transfer under a current of 300A. A large droplet forms with electric potential and velocity distributions similar to the case representing globular transfer at a current of 160A.

\subsection{Summary}

A numerical method employing advanced techniques in CFD has been applied to simulate the dynamic process of metal transfer in constant current GMAW. The current density on the droplet surface has significant effect on the electromagnetic force calculation. The assumption of Gaussian current density distribution on the droplet surface is shown to be a good approximation, which produces calculated results closer to experimental measurements than other assumptions. The detaching-droplet size predicted using Gaussian assumption not only shows very good consistency with the experimental data for globular and spray transfer, but also for the transition range between globular and spray transfer. Effects of surface tension, electromagnetic and gravitational force, which dominate the dynamic character of droplet development and therefore influence the metal transfer mode, are analyzed. The numerical analysis provides a good understanding of the physical mechanisms that dominate the metal transfer process under different current ranges in GMAW. It not only explains the globular metal transfer with large droplets detached by a balance of gravity and surface tension and spray metal transfer with small 
droplets detached by a balance of electromagnetic force and surface tension, but also gives the observation of bifurcation in droplet size under the transition current range its numerical interpretation. The present study is the first to accurately estimate the transition current and explain the unique metal transfer behavior during the transition of metal transfer mode.

Copyright ( Ge Wang 2007 


\section{Chapter 5}

\section{Metal Transfer in Pulsating Current GMAW}

In this chapter, the metal transfer process in pulsed current GMAW is simulated and analyzed by using our numerical program. Therefore, the optimum operational conditions to achieve the desirable one drop per pulse (ODPP) metal transfer mode can be determined in systematic ways. A novel modified pulsed current GMAW is proposed to improve the robustness of the welding process to realize ODPP metal transfer. In the proposed approach, a droplet oscillation induced by an exciting current pulse brings downward momentum. A droplet is detached by the combined effects of downward momentum and increased electromagnetic force provided by a detaching current pulse. The utilization of downward momentum eliminates the need for a very high current to detach the droplet every pulse cycle, which is required in conventional pulsed current GMAW. This elimination guarantees that no unexpected detachment occurs during droplet growth and extends the application of a pulsed GMAW in thin sectioned or heat sensitive materials. The predicted results are in good agreement with the experimental data. The phase match between the droplet oscillation and the current increase, a key for achieving desirable ODPP metal transfer under this modified pulsed current GMAW, can be diagnosed by numerical calculation. The welding operational parameters and their 
ranges are also calculated and the predicted results further demonstrate the robustness of this new GMAW technique in achieving high quality welding.

\subsection{Introduction}

As it has been discussed in chapter 4, globular transfer and spray transfer are two basic metal transfer modes under constant current GMAW. Globular transfer mode typically brings significant spatters and a broad weld pool, and therefore results in poor welding quality [12]. Spray transfer mode, which can be further classified into drop spray and streaming spray, has advantages over the globular mode due to its regular detachment with uniform droplet size, directional droplet transfer and low spatters [13]. Streaming spray takes place under a higher current with a high frequency of detached droplets having much smaller diameters than the electrode. A streaming spray transfer causes high-speed impact of droplets into the weld pool and results in poor mechanical properties [3]. Drop spray, which occurs under a relatively low current range with the diameter of detached droplet almost the same as the electrode, is often being preferred [48]. However, drop spray still requires a current higher than the transition current, which sometime results in a thermal load too high to apply to thin sectioned or heat-sensitive materials.

Pulsed current GMAW was introduced during 1962 [14] as an intermediate mode. The current used in this mode is shown in Figure 5.1 with a relatively low background current (base current $I_{b}$ ) interrupted by pulses of high current (peak current $I_{p}$ ). Pulsed 
current GMAW uses a low base current $I_{b}$ to maintain the arc and a high peak current $I_{p}$ to melt the electrode and detach the droplet. By using pulsed current GMAW, a controlled drop spray transfer mode can be achieved at low average current that typically results in globular transfer. But on the other hand, the pulse introduces additional parameters including the peak current $I_{p}$, the base current $I_{b}$, the peak duration $T_{p}$ and the base duration $T_{b}$. Depending on the combinations of these parameters, metal transfer could happen in three different ways under pulsed current GMAW. Multi-drops per pulse (MDPP) occurs when there is more than one droplet detached during a pulsing cycle. One drop multiple pulses (ODMP) occurs when the droplet detachment does not occur every pulse. One drop per pulse (ODPP) with a single droplet detached every pulse, which characterizes a stable, periodical, and controllable metal transfer process, produces the best weld quality and is the most desirable metal transfer mode.

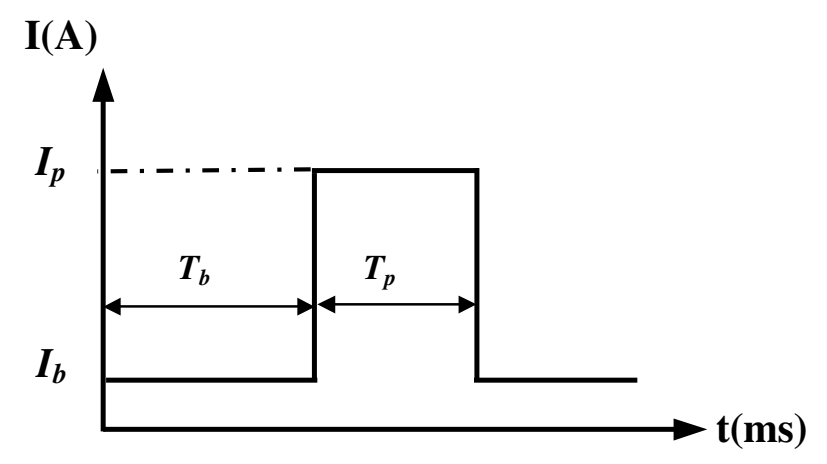

Figure 5.1 Current waveform for conventional pulsed GMAW

The necessary conditions to achieve the desirable one drop per pulse (ODPP) mode were investigated in a number of works [48-59]. It has been pointed out by Ueguri 
[48] and Amin [49] that the peak current $I_{p}$ should be set above a current threshold, which is the transition current between the globular and spray transfer mode, to obtain the ODPP mode. A peak current above the transition current has the drawbacks of easily bringing accidental detachment, i.e. multiple droplets detached per pulse (MDPP), and overheating the droplet and welding pool.

Recently, a novel active control technology has been proposed by E Zhang and his associates [63-65] in order to achieve ODPP metal transfer under a lower peak current. A droplet is detached by the combination of the downward momentum of the drop oscillation and the increased electromagnetic force, which is induced by an exciting pulse and a detaching pulse, respectively. The synchronization between the downward movement and increased current must be satisfied to ensure ODPP when using this new technology. This method introduces a large number of additional welding parameters, which make it difficult to select optimum combinations of parameters for a wide range of welding conditions. The experimental determination of these parameters under different welding conditions is laborious and unfeasible.

A theoretical description of the metal transfer in GMAW can provide a better understanding of the technology's mechanism and a better means to determine the optimum operational parameters. Some numerical studies have been done for constant current and for traditional pulsed current GMAW [25-57]. Such studies have developed from the earliest static models to the dynamic models. In this work, a transient twodimensional model developed based on RIPPLE [60] is used to simulate droplet 
formation, detachment, and transport in GMAW. The transient shape of the droplet is calculated using the fractional volume of fluid (VOF) method [61]. The continuum surface force (CSF) model [62] used here simplifies the calculation of surface tension and enables accurate modeling of fluid flows driven by surface forces. The electromagnetic force, which is generated by the welding current, is calculated by assuming Gauss-type current density distribution over the free surface of the droplet. The numerical calculation was successfully applied to constant current GMAW in the Chapter 4.

In this chapter, the calculation is first carried out for conventional pulsed current GMAW. The effects of the peak current and pulse duration on droplet detachment are analyzed and the predicted results are compared with the available data. Then, numerical simulation and analysis are performed for the metal transfer in the modified pulsed current GMAW. The parameters are diagnosed for phase match in order to achieve ODPP metal transfer under the modified pulsed current GMAW. The operating ranges of various pulse signal parameters including the time interval $T_{b 2}$, exciting pulse duration $T_{p 1}$, peak current $I_{p}$ and pulse frequency $f$ have been under scrutiny. The numerical calculation will not only demonstrate the effectiveness of the proposed approach in achieving the desired metal transfer process, but will also provide a theoretical means to determine the operational parameters. 


\subsection{Proposed approach in modified pulsed current GMAW}

In conventional pulsed GMAW using a pulse waveform as shown in Figure 5.1, the droplet is supposed to be detached by the pulsed current. The detachment of a droplet is still considered as a natural detachment process driven only by the combined effects of gravitational force and electromagnetic force. For a current lower than the transition current, a natural detachment can only occur when the diameter of droplet is significantly larger than that of the electrode. Hence, the peak current $I_{p}$ must be higher than the transition current in order for the droplet to naturally detach with a size similar to the diameter of the electrode $[48,49]$. On the other hand, the use of a peak current higher than the transition current narrows the range of the peak current duration for generating stable ODPP metal transfer. If the duration of the peak current period is longer than required, multiple droplets may be detached in a single pulse, which brings accidental detachment and causes multiple-droplets-per-pulse (MDPP) [69]. If the duration is shorter, multiple pulses may be needed to develop and detach one droplet, which causes one-droplet-multiple-pulses (ODMP) [54].

To obtain ODPP metal transfer with a droplet size similar to the diameter of electrode, while the peak current is lower than the transition current to prevent accidental detachment, a novel active control technology has been recently proposed [63-65]. In this modified pulsed current GMAW, a pulse cycle is composed of two periods: growth period and detachment period as shown in Figure 5.2. An exciting pulse edge is applied at the end of the growth period when the current is switched to the base level so that a 
sudden change in electromagnetic force is imposed to the droplet. As a result, an oscillation of the droplet is introduced. After a period $T_{b 2}$ as shown in Figure 5.2, a detaching pulse is applied. The downward momentum of the oscillating droplet enhances detachment and eliminates the need for a higher current to detach the droplet. (The detachment of the droplet is no longer a natural transition in this proposed approach but a controlled result.) Hence, the peak current can be lower than the transition current to detach the droplet while at the same time accidental detachment is prevented. Also, the elimination of the use of high current reduces superheating related fumes, material property damage and burning-through of thin-sectioned material.

From the parameters of modified pulsed current GMAW shown in Figure 5.2,

$$
\begin{aligned}
& T_{b}=T_{b 1}+T_{b 2} \\
& T_{p}=T_{p 1}+T_{p 2} \\
& T=T_{b 1}+T_{b 2}+T_{p 1}+T_{p 2}=T_{b}+T_{p}=1 / f \\
& I_{a v g}=\left(I_{p} * T_{p 1}+I_{p} * T_{p 2}+I_{b} * T_{b 1}+I_{b} * T_{b 2}\right) / T=\left(I_{p} * T_{p}+I_{b} * T_{b}\right) * f
\end{aligned}
$$

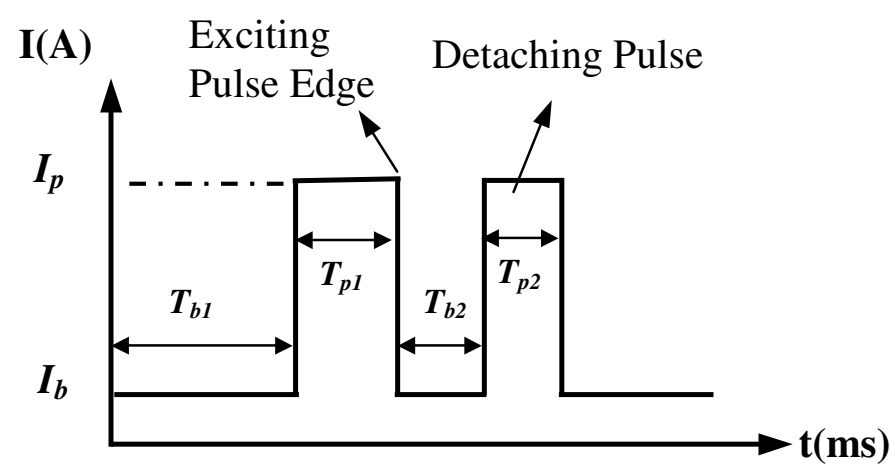

Figure 5.2 Current waveform used for modified pulsed GMAW 
where $f$ is the pulse frequency, $I_{\text {avg }}$ is the average current, $I_{p}$ is the peak current, $I_{b}$ is the background current, and $T_{p}$ and $T_{b}$ represent the peak and base duration respectively. If any four of these are given as preset parameters, the other two parameters can be determined accordingly. The waveform of the welding current can be changed by the adjustment of $T_{p 1}, T_{p 2}, T_{b 1}$ and $T_{b 2}$ without change of the preset parameters. By properly choosing the waveform of the welding current, the proposed method takes advantage of synchronization between the downward momentum of the droplet oscillation and the increased electromagnetic force provided by detaching pulse to realize ODPP metal transfer. The synchronization between the downward momentum of the droplet and the increase of the detaching electromagnetic force is referred to as phase match. The phase match is the key to achieve ODPP metal transfer when using this modified pulsed current GMAW technique.

Experiments have demonstrated the effectiveness of the proposed approach [6365]. But a significant limitation of the control system developed by experiment is the use of a high-cost imaging system, which monitors the oscillating droplet to provide the phase match. Since this new technique brings in many parameters, the experimental determination of the operational combination of these parameters to reach phase match and therefore realize ODPP metal transfer is laborious and costly. Numerical simulations can provide a better understanding of the mechanism of this process and the means to determine the optimum operation parameters. 
As mentioned earlier, the proposed approach takes advantage of the downward momentum of the excited droplet oscillation to reduce the current level for the droplet detachment and prevent accidental detachment. In order to achieve the detachment and realize ODPP, the phase match between the downward momentum and the detaching action is crucial. The proper selection of $T_{b 2}$, the time interval between the exciting pulse and detaching pulse, determines whether or not the phase match condition between the downward momentum and the detaching action can be met. Because of the importance of the time interval $T_{b 2}$, a numerical solution is introduced here to determine it and guarantee phase match. Since the oscillation of the droplet is induced by exciting pulse, the excited oscillation of the droplet is numerically simulated first. Based on analysis of the calculated results, the proper time interval $T_{b 2}$ is then determined to assure phase match and obtain ODPP metal transfer.

\subsection{Numerical Results and Discussions}

Calculations were performed based on the experimental work of Zhang and his associates $[63,64]$. Most simulations were carried out for stainless steel electrodes with a diameter of $1.2 \mathrm{~mm}$. The physical properties of the stainless steel electrodes are listed in Table 1. A uniform computational mesh with a spacing of $0.1 \mathrm{~mm}$ in each coordinate direction was used.

Since the transition current range between globular mode and spray mode plays a critical role in the study of metal transfer, the calculations are performed for the metal 
transfer process under constant current to predict the transition current range first. Figure 5.3 shows the average droplet sizes predicted using our numerical program for different welding currents while the wire feed rate is set to be $70 \mathrm{~mm} / \mathrm{s}$. As the welding current increases, the metal transfer mode changes from the globular mode with a large droplet detached to the spray mode with a small droplet detached. The results once again support the existence of a narrow current range (transition current range) over which a remarkable change from the globular transfer mode to the spray transfer mode occurs. Hence, the transition current range for the stainless steel electrode with diameter of $1.2 \mathrm{~mm}$ is predicted to be from $220 \mathrm{~A}$ to $230 \mathrm{~A}$ according to the calculations.

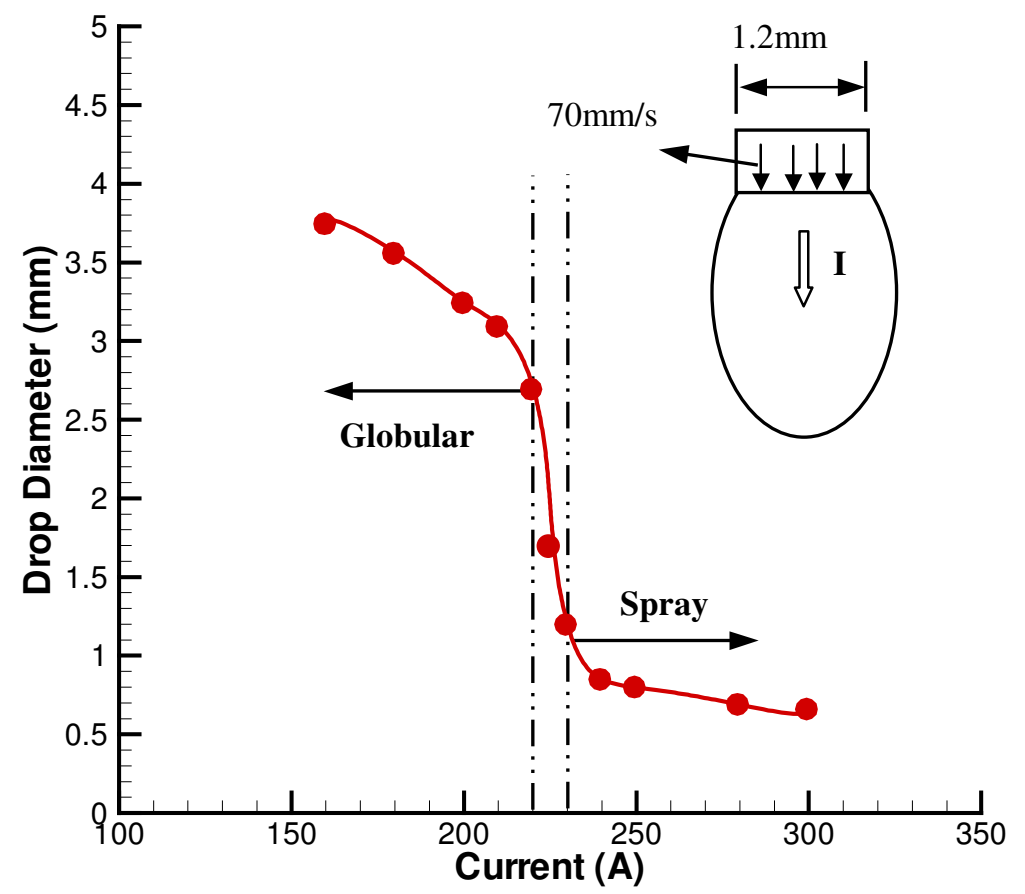

Figure 5.3 The relationship between the detached drop sizes and welding currents 


\subsubsection{Traditional Single Pulsed Current GMAW}

Traditional single pulsed current GMAW has an advantage over the constant current GMAW due to the possibility of achieving a stable spray metal transfer mode (one drop per pulse mode) at low average current that would otherwise bring globular transfer with large sporadic droplets. The lower average current reduces the thermal load on the weld to avoid overall overheating. Also, GMAW under the pulsed current mode can produce fewer fumes as Castner showed in his paper [70]. Hence, pulsed current GMAW has been widely used in mechanized or robotic welding ever since it was introduced. However, the droplets detached in traditional single pulse current GMAW are due to increased electromagnetic force during the peak current period. It is still a natural detachment process as the electromagnetic force and gravity are still the only forces to detach a droplet. The peak current $I_{p}$ must be higher than the transition current in order to ensure that the droplet naturally detaches in the spray mode with a smaller droplet size.

On the other hand, the use of a peak current higher than the transition current can easily cause accidental detachment and multiple-droplets-per-pulse (MDPP) metal transfer. Calculations were performed and the results were consistent with the above analysis.

The dynamic drop development and detachment processes under traditional single pulsed current GMAW were simulated. Three different kinds of metal transfer cases are presented here, which represent the desired one drop per pulse (ODPP) under low average current, one drop multiple pulses (ODMP) with undesired larger droplet detached under peak current lower than transition current and Multi-drops per pulse (MDPP) with 
unwanted accidental detachment under peak current higher than transition current, respectively.

For the case representing ODPP metal transfer, the calculation was carried out for a steel electrode of $1.6 \mathrm{~mm}$ diameter for an average current of $202 \mathrm{~A}$ when the peak current $I_{p}$ was $400 \mathrm{~A}$ and base current $I_{b}$ was set to $180 \mathrm{~A}$. The wire feed speed was set to be $45 \mathrm{~mm} / \mathrm{s}$. When the pulsing frequency was $20 \mathrm{~Hz}$, metal transfer with one drop per pulse was observed. The calculated dynamic variations of the droplet profile and vertical coordinate of droplet tip with current pulses are shown in Figure 5.4. The instants for droplet detachment are marked with dots in the figure.

Since this set of data was taken from the pervious study of Choi, Yoo and $\operatorname{Kim}[54,56]$, the broad agreement between our calculations and their experimental results provides a primary test for pulsed current GMAW simulation performed using our program. Calculations were also carried out for a range of the pulsing frequency, with the other parameters held constant, to determine the operating range of the pulsing frequency that provides ODPP metal transfer. When the droplet detaching to current pulse frequency ratio is equal to one, one drop per pulse metal transfer occurs. Figure 5.5 shows comparison of the calculated ratios between the droplet detaching and current pulse frequency with available data. The calculated data provided by the present study are marked with crosses in the figure. Our predicted results are compared with the experimentally data as well as the results calculated by Choi, Yoo and Kim. It is notable 
that the present calculated results show better agreement with the experimental data than the results provided by Choi, Yoo and Kim.
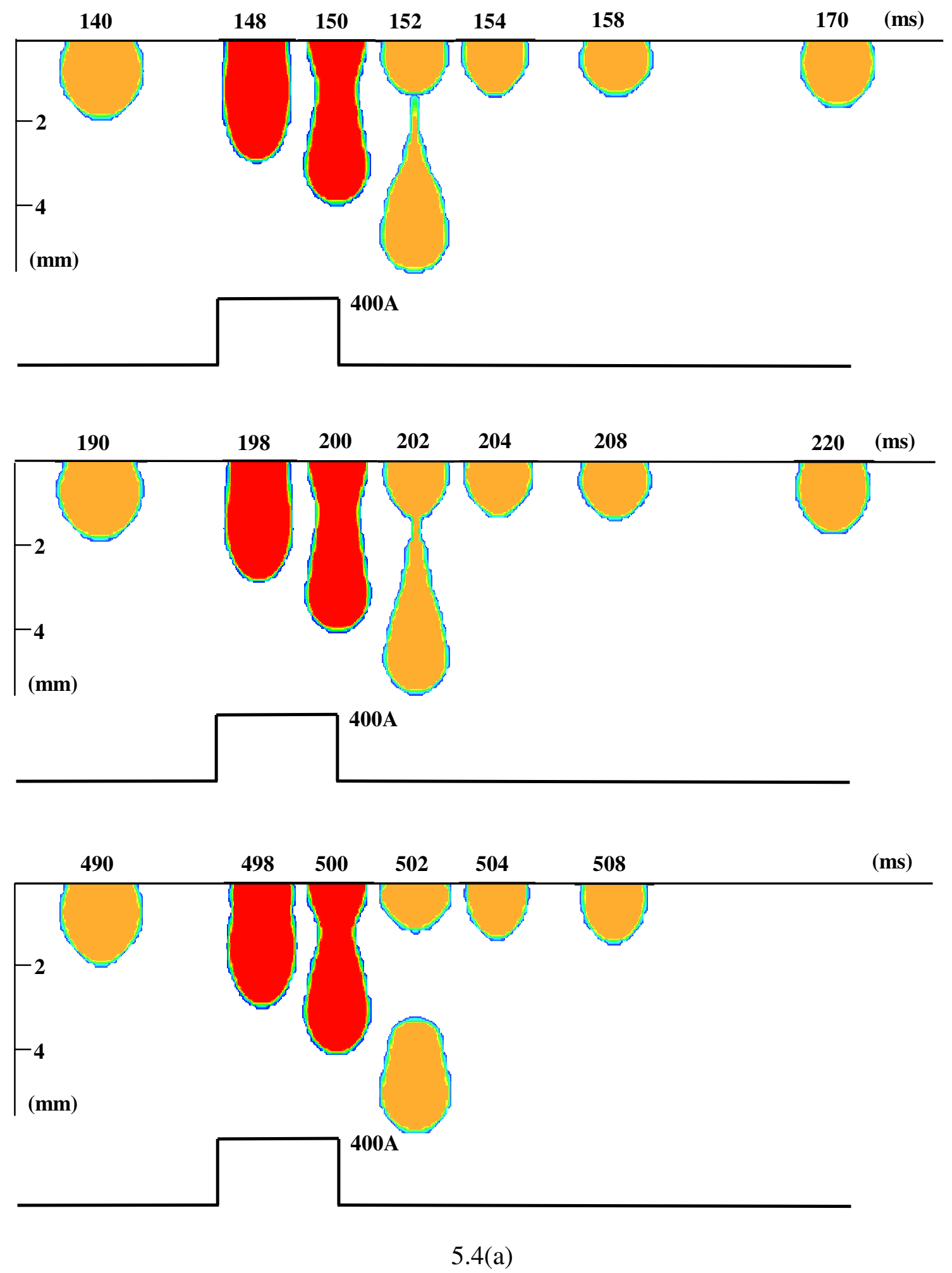


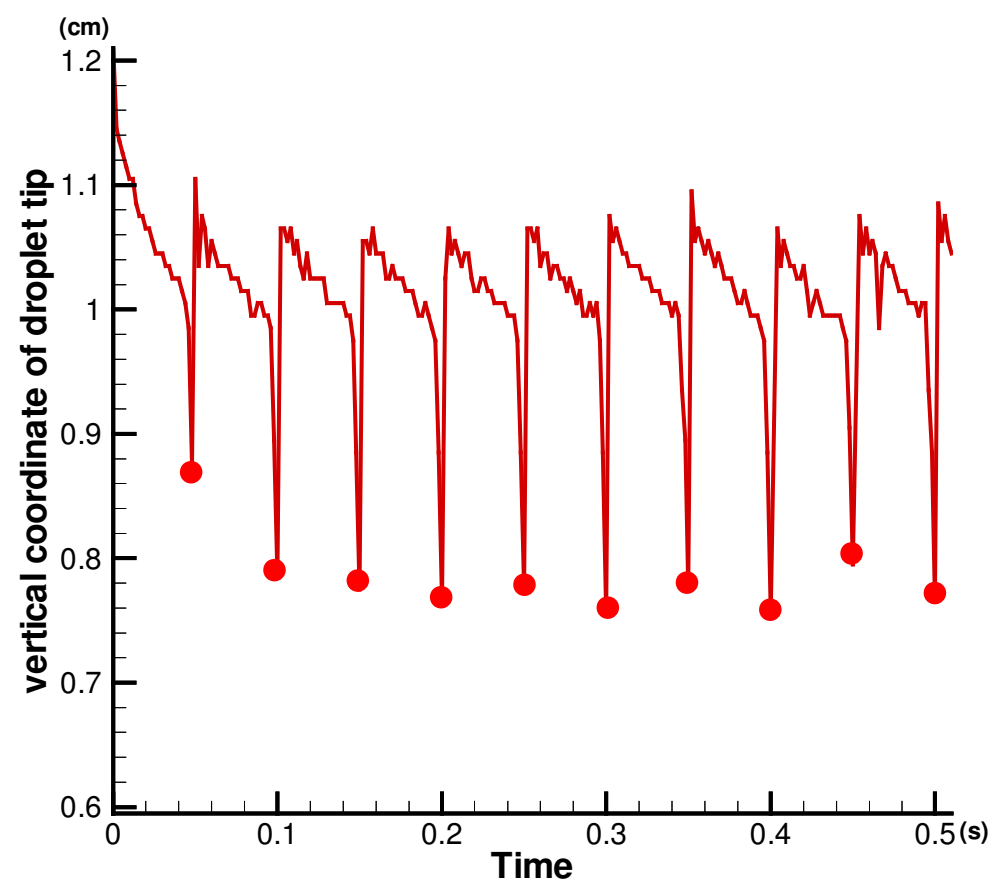

$5.4(\mathrm{~b})$

Figure 5.4 Metal transfer with ODPP under conventional pulsed current GMAW:

(a) Droplet profiles (b) Vertical coordinate of droplet tip

For the case representing ODMP metal transfer, the calculation was carried out for a steel electrode with a diameter of $1.2 \mathrm{~mm}$. The average current $I_{\text {avg }}$ is set as 100A, the pulse frequency $f$ is $30 \mathrm{~Hz}$, the base current $I_{b}$ is $40 \mathrm{~A}$ and peak current $I_{p}$ is $220 \mathrm{~A}$ (lower than the transition current range). According to experimental data taken from the work of Zhang, E, and Walcott [63], the wire feed rate is selected to be $110 \mathrm{in} / \mathrm{min}$ $(46.5 \mathrm{~mm} / \mathrm{s})$ for an average current around 100A. The calculated dynamic variations of the droplet profile with current pulses are shown in Figure 5.6. Metal transfer with one drop multiple pulses (ODMP) can be visualized. The predicted results support the general idea 
that the peak current must be higher than the transition current in order to ensure that the droplet is detached by each pulse with a size similar to the diameter of electrode in conventional single pulsed current GMAW. Also, the droplet oscillation introduced by the current pulse can be easily visualized from Figure 5.6. It shows the instantaneous profiles for droplet bouncing to the highest position immediately after a current pulse drags it down. It can be noted the time required for the droplet to reach the highest position after a current pulse is increased with increasing mass of droplet. In other words, the frequency of droplet oscillation after a current pulse is decreased with an increase in droplet mass.

The metal transfer process was simulated as the peak current $I_{p}$ increased to $250 \mathrm{~A}$, which is higher than the transition current. The other welding conditions, which include the base current, the base duration, the peak duration, and the pulse frequency, were kept the same as the above ODMP metal transfer case. The calculated dynamic profile variations of the droplet are illustrated in Figure 5.7. This situation easily causes accidental detachment while generating more than one droplet during one current pulse cycle. Hence, metal transfer with undesired Multi-drops per pulse (MDPP) occurs. Also, the use of high current causes superheating related fumes, material property damage and burning-through of thin-sectioned material.

In order to overcoming the drawbacks associated with the requirement of a peak current higher than transition current in conventional pulsed current GMAW, a modified pulsed current GMAW has been proposed to achieve the desired metal transfer. 


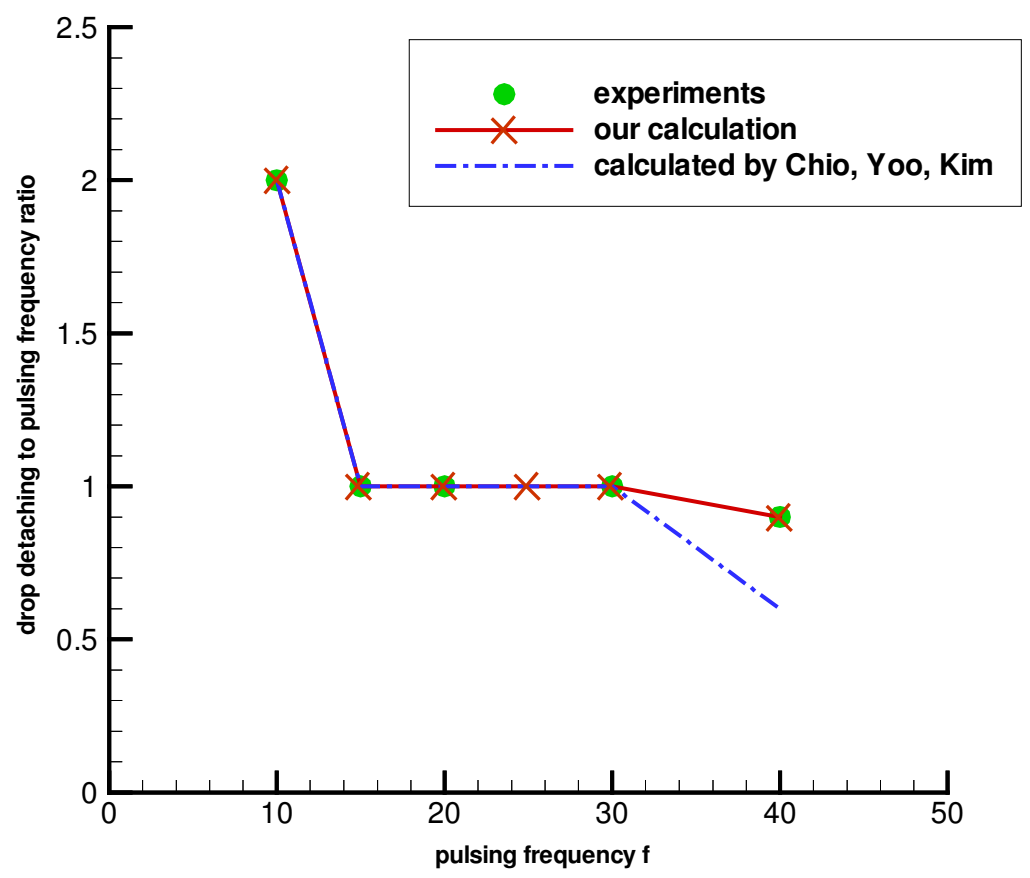

5.5(a)

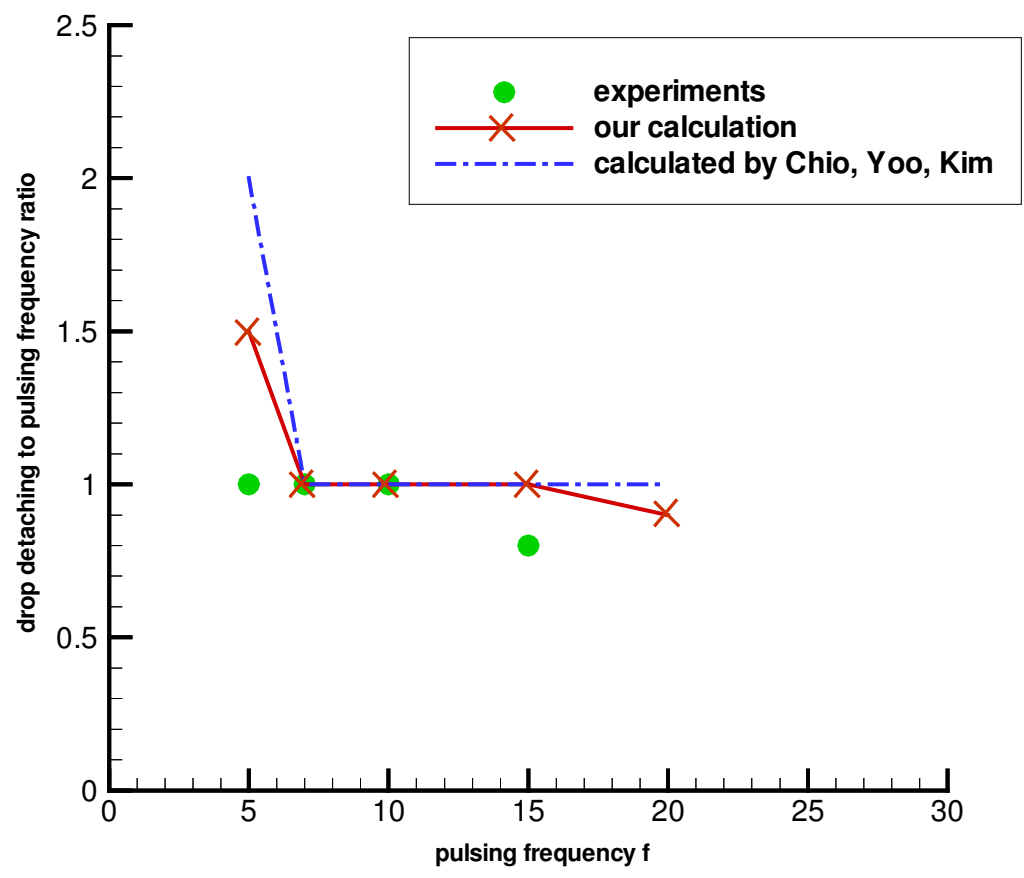

5.5(b) 


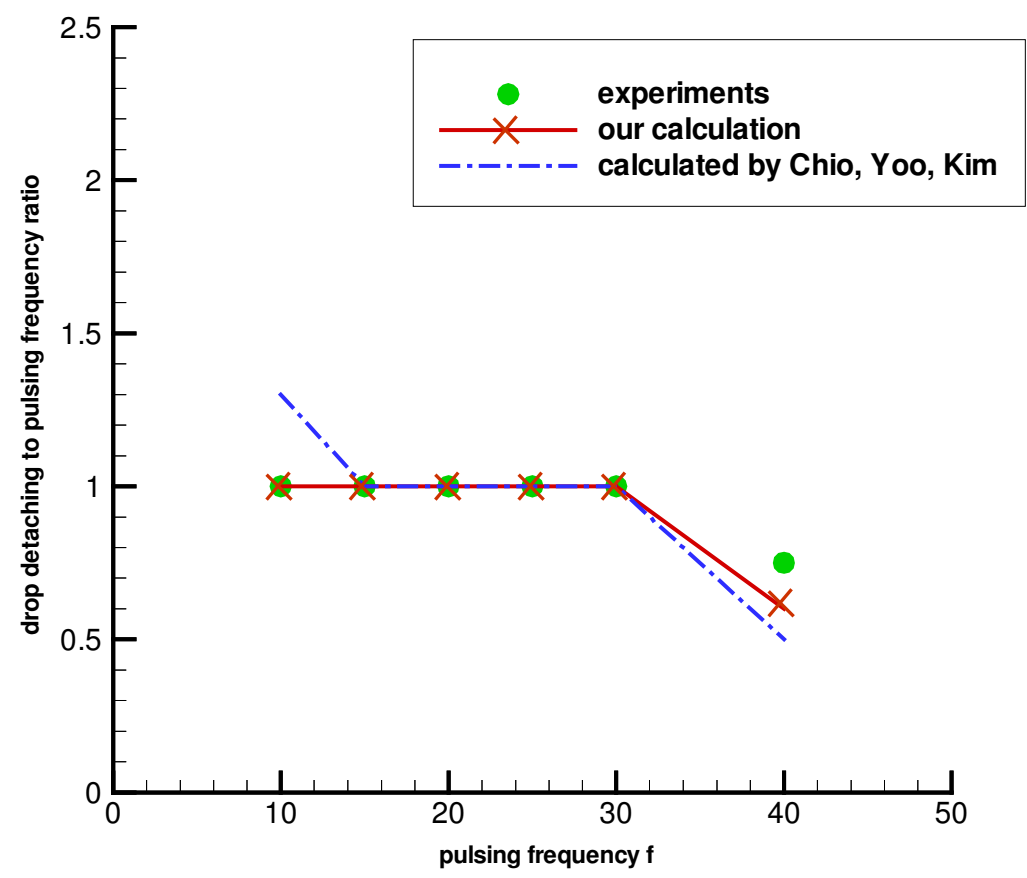

5.5(c)

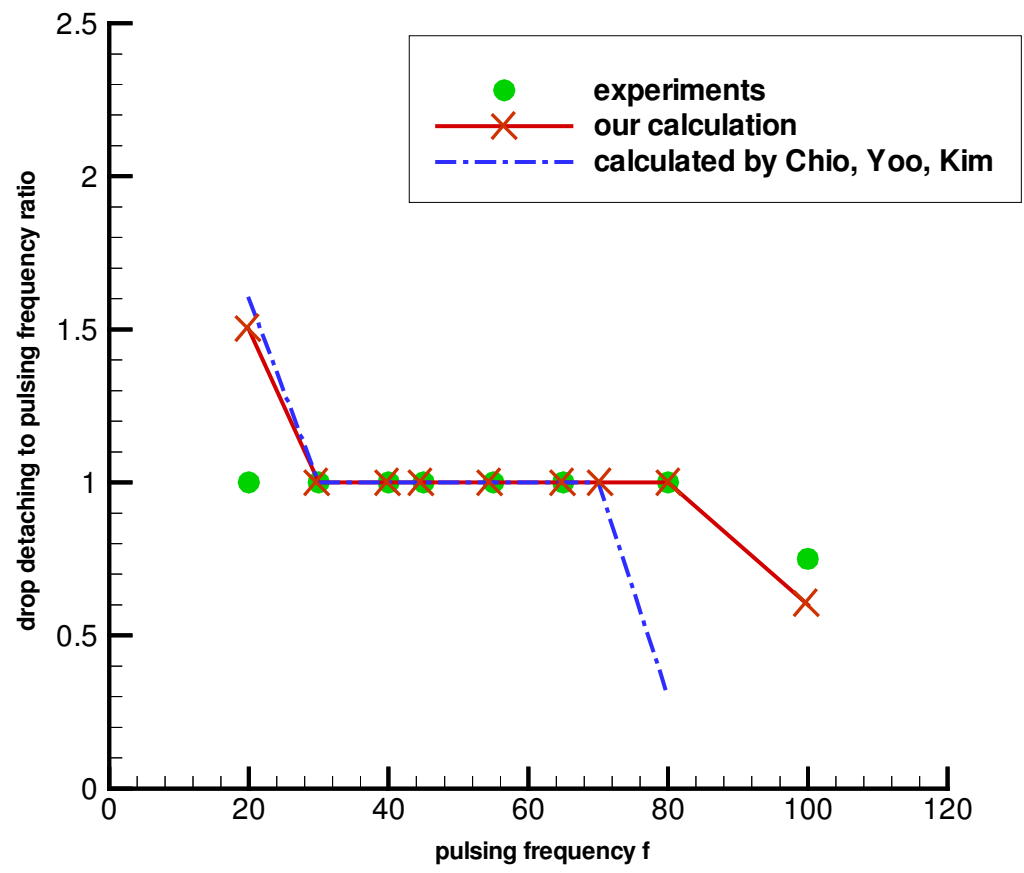

5.5(d)

Figure 5.5 Comparison of calculated and experimental operating ranges:
a. $I_{p}=400 \mathrm{~A}, I_{b}=180 \mathrm{~A}, I_{a v g}=202 \mathrm{~A}$
b. $I_{p}=400 \mathrm{~A}, I_{b}=180 \mathrm{~A}, I_{a v g}=191 \mathrm{~A}$

c. $I_{p}=500 \mathrm{~A}, I_{b}=180 \mathrm{~A}, I_{a v g}=196 \mathrm{~A}$

d. $I_{p}=500 \mathrm{~A}, I_{b}=180 \mathrm{~A}, I_{a v g}=212 \mathrm{~A}$ 

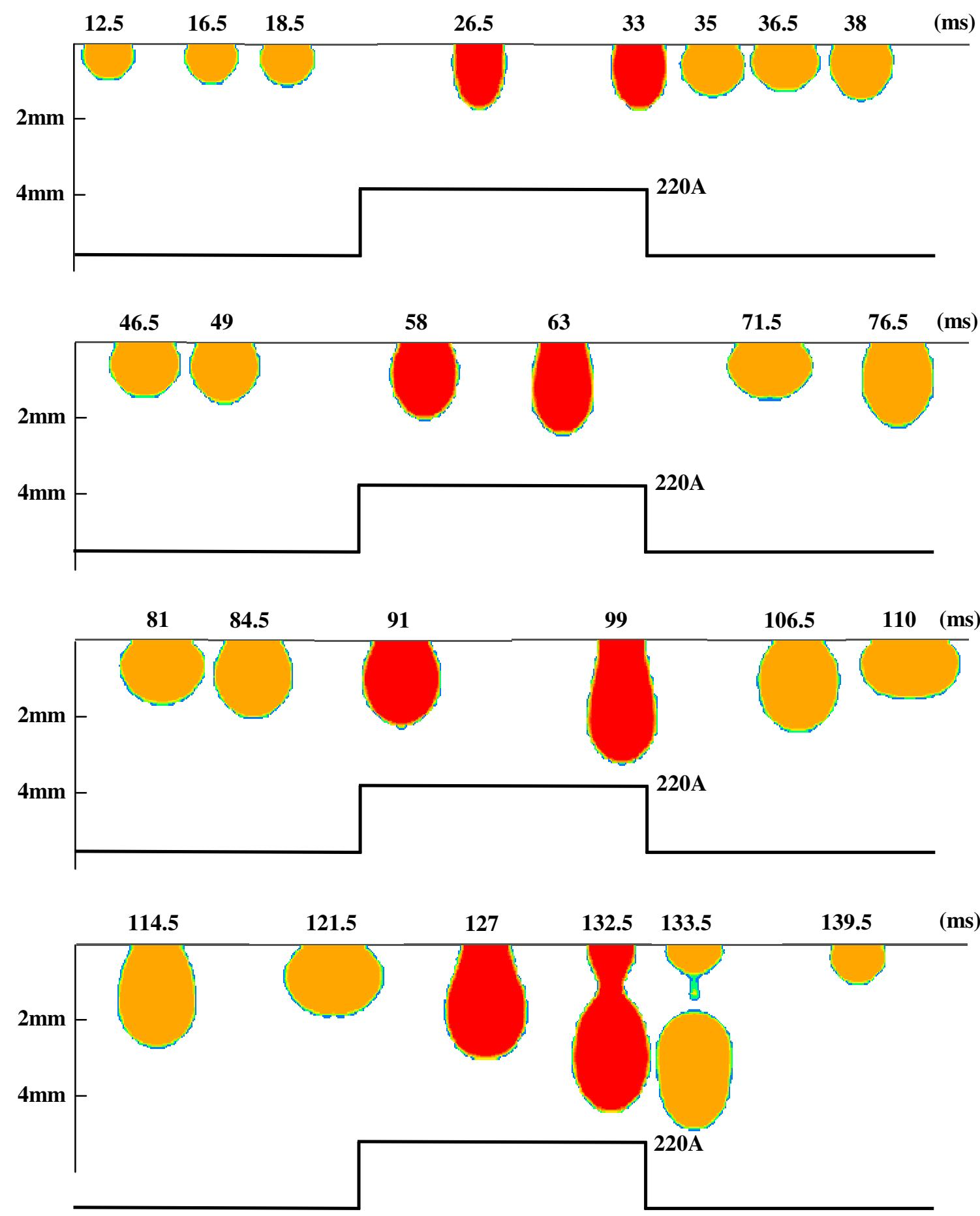

Figure 5.6 Metal transfer with ODMP under conventional pulsed current GMAW 

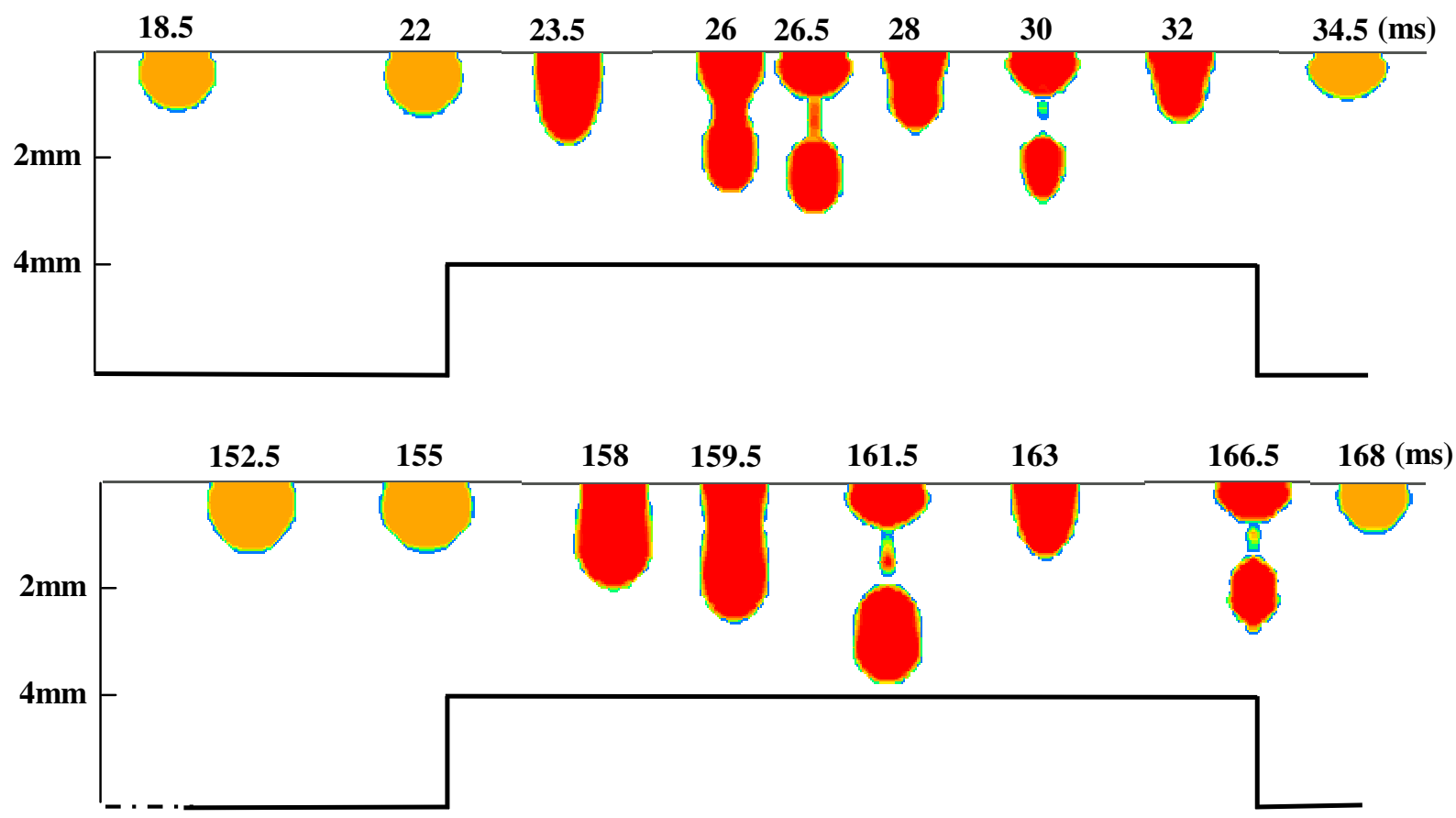

Figure 5.7 Metal transfer with MDPP under conventional pulsed current GMAW

\subsubsection{Modified Pulsed Current GMAW}

By simply splitting the current pulse used in the above ODMP case into two parts and keeping all other parameters unchanged, the current signal used in the modified pulsed current GMAW is shown in Figure 5.8(a). The average current $I_{\text {avg }}$ is 100A, the pulse cycle frequency $f$ is $30 \mathrm{~Hz}$, the base current $I_{b}$ is $40 \mathrm{~A}$, the peak current $I_{p}$ is $220 \mathrm{~A}$, the exciting pulse duration $T_{p 1}$ is $5 \mathrm{~ms}$, the detaching pulse duration $T_{p 2}$ is $6 \mathrm{~ms}$ and 
the time interval between the exciting pulse and the detaching pulse $T_{b 2}$ is set as $4 \mathrm{~ms}$. The calculated dynamic variations of the droplet profile with current pulses are shown in Figure 5.8(b). The droplet oscillation can be visualized. The droplet bounces upward immediately after an exciting pulse, because of the sudden change in welding current resulting in the dramatic decrease of electromagnetic force. The detaching pulse is added when the droplet reaches its highest position and starts to work its way down, which in this case occurs $4 \mathrm{~ms}$ after the exciting pulse. With the assistance of the oscillating droplet's downward momentum, the increased electromagnetic force detaches the droplets with a detaching pulse current lower than the peak current demand in traditional pulsed current GMAW.

The calculated dynamic development of the droplet tip vertical coordinate is shown in Figure 5.8(c) with the instances of droplet detachment marked with dots. It shows that the one droplet per pulse metal transfer is achieved with a droplet size similar to the diameter of the electrode under the peak current of $220 \mathrm{~A}$, which is lower than the transition current, by using the modified pulsed current GMAW. The calculation proves the principle of the proposed approach: the modified pulsed current GMAW takes advantage of the phase match between the downward momentum of the oscillating droplet introduced by the exciting pulse and the increased electromagnetic force brought by the detaching pulse; The current level, which is required to detach a droplet each pulse cycle with a size similar to the diameter of the electrode, is lowered by introducing the downward momentum of the oscillating droplet. This test case is named as the model case for easy reference later. 


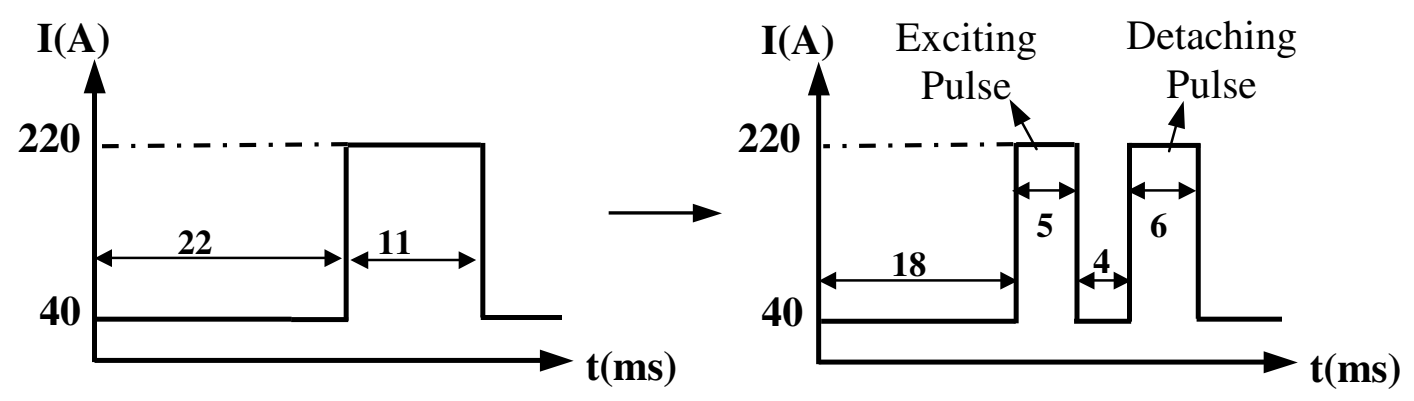

5.8 (a)
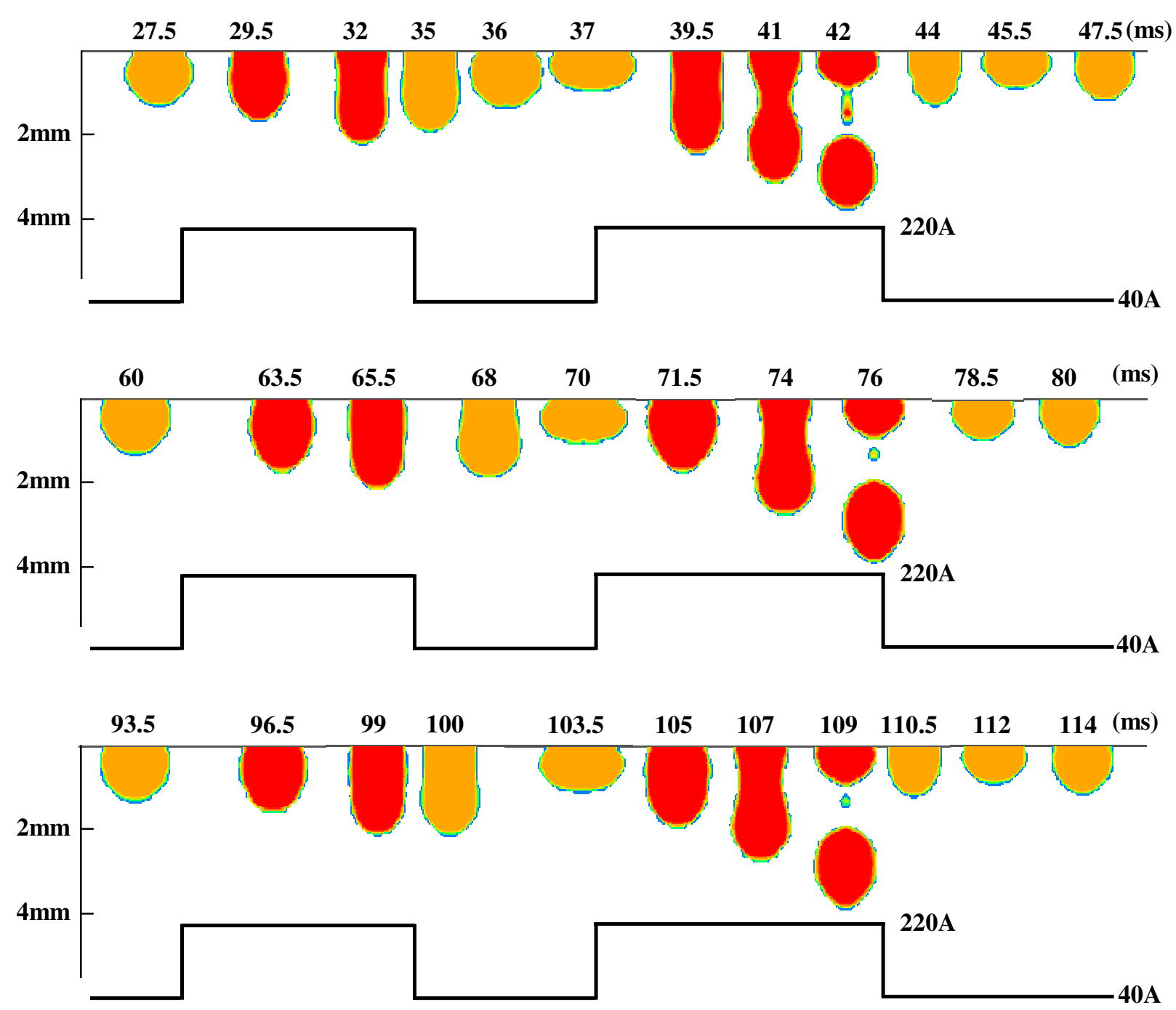

5.8 (b) 


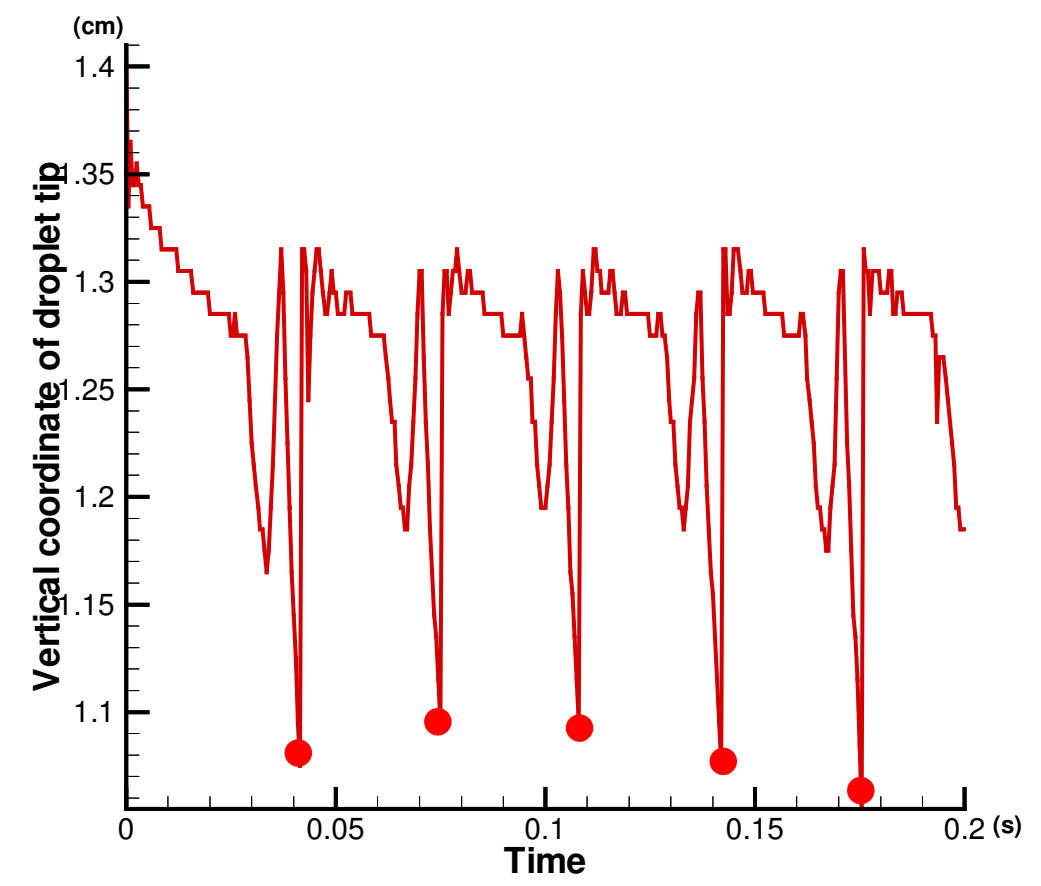

$5.8(\mathrm{c})$

Figure 5.8 Metal transfer with ODPP under modified pulsed current GMAW:

(a) Current waveform (b) Droplet profiles (c) Vertical coordinate of droplet tip

\subsubsection{Parameter Diagnoses for Phase Match}

As it can be observed in Figure 5.8, when the time interval between the exciting pulse and detaching pulse $T_{b 2}$ is selected to be $4 \mathrm{~ms}$, ODPP metal transfer is realized. However, if a shorter time interval $T_{b 2}=3 \mathrm{~ms}$ or a longer time interval $T_{b 2}=6 \mathrm{~ms}$ is used and other parameters including the average current $I_{\text {avg }}$, pulse cycle frequency $f$, peak current $I_{p}$, base current $I_{b}$ and exciting pulse duration $T_{p l}$ are kept the same as the model case, the ODPP metal transfer cannot be realized as shown in Figure 5.9 and Figure 5.10. 
In the above two cases, the failure of ODPP metal transfer is caused by the unsatisfied phase match condition.
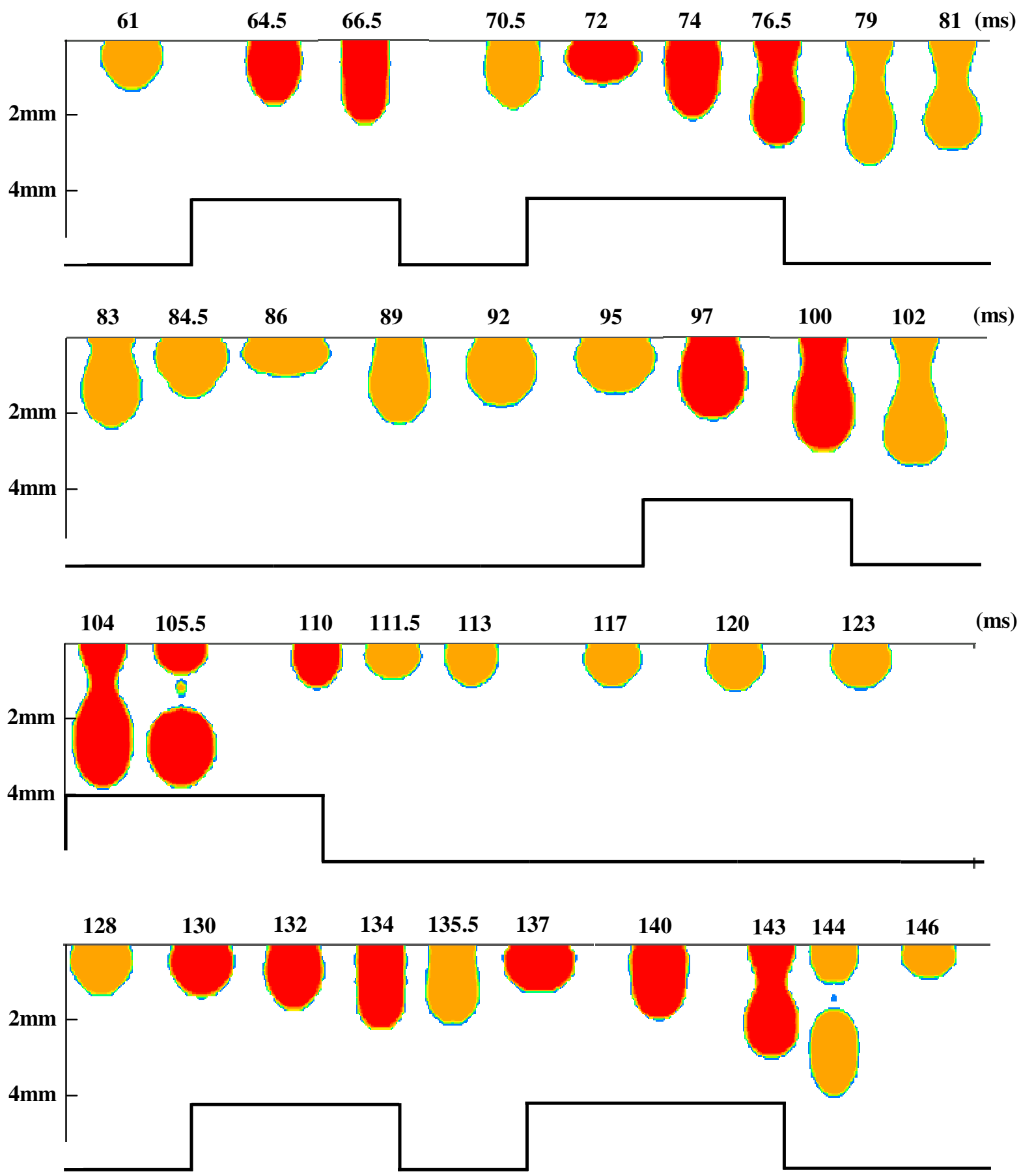

$5.9(\mathrm{a})$ 


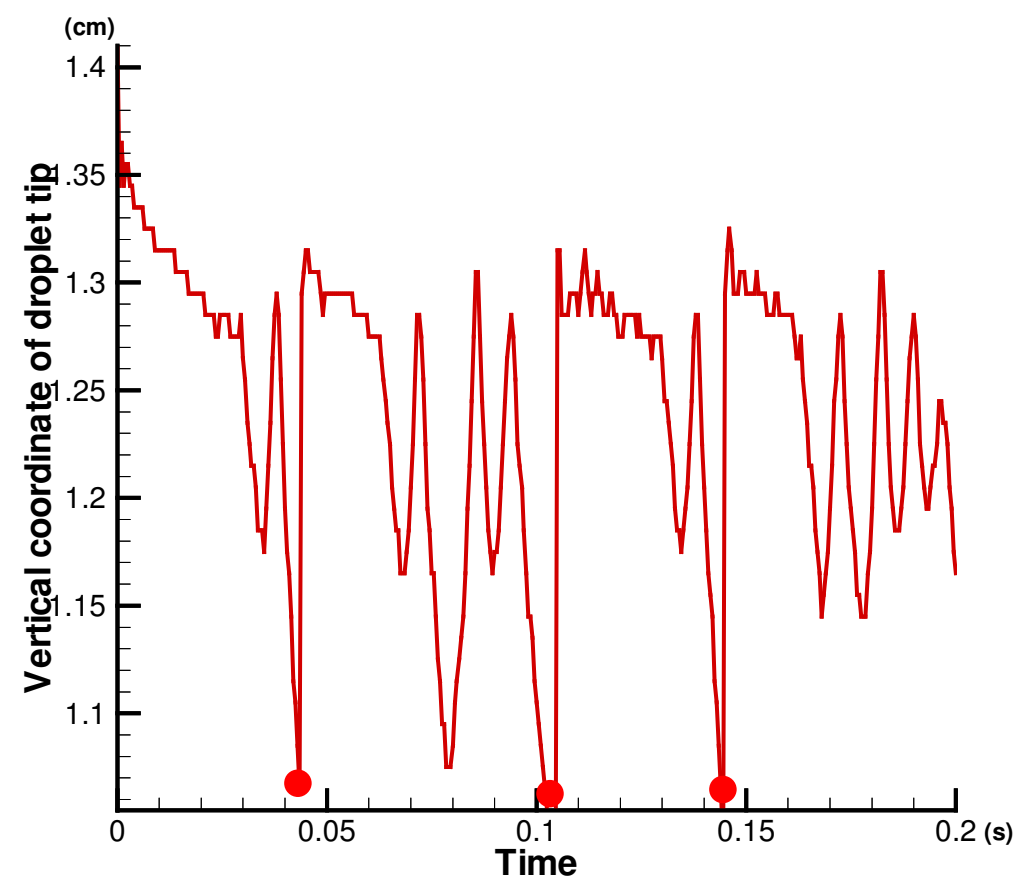

5.9 (b)

Figure 5.9 Metal transfer processes with unsatisfied phase match condition:

(a) Drop profiles under a shorter duration $T_{b 2}$ of $3 \mathrm{~ms}$

(b) Vertical coordinate of droplet tip

In the first case, the calculated dynamic variations of the droplet profile with current pulses and dynamic development of the droplet tip vertical coordinate are shown in Figure 5.9. The time interval of $3 \mathrm{~ms}$, between the exciting pulse and the detaching pulse, is too short to let the droplet bounce to its highest position before the detaching pulse arrives. Hence, the droplet continues moving to the higher position during part of the period of the detaching pulse application. The upward momentum of droplet not only cannot reinforce the detaching process, but also offsets the electromagnetic force and makes the droplet detachment process more difficult. 

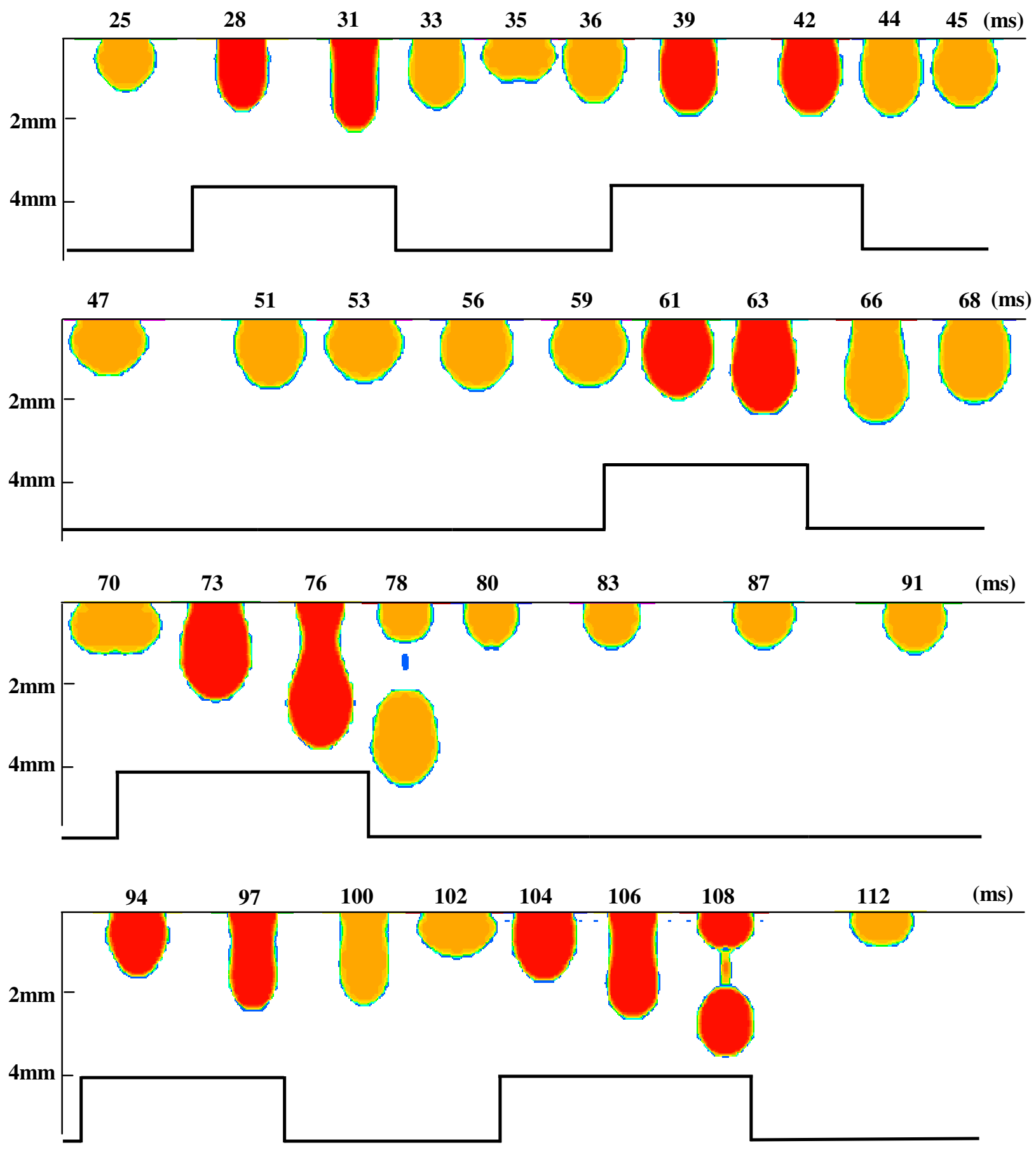

5.10 (a) 


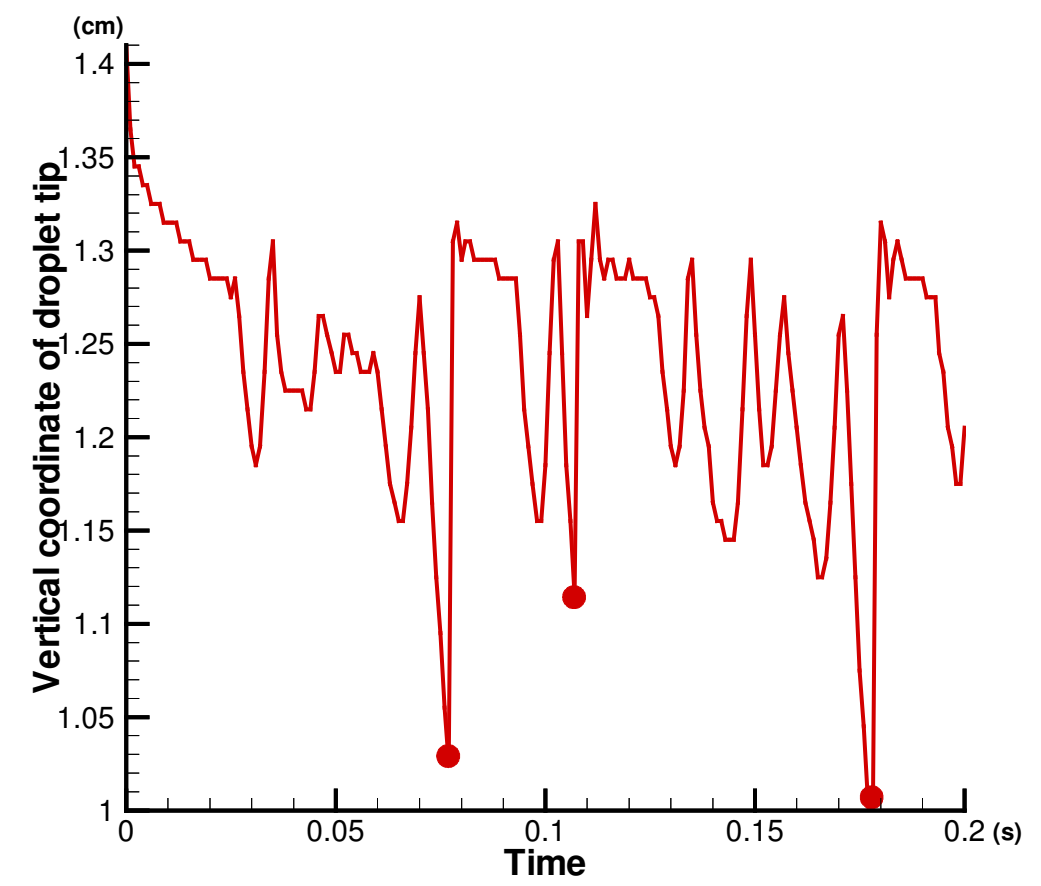

$5.10(b)$

Figure 5.10 Metal transfer processes with unsatisfied phase match condition:

(a) Drop profiles under a longer duration $\mathrm{T}_{\mathrm{b} 2}$ of $6 \mathrm{~ms}$

(b) Vertical coordinate of droplet tip

In the second case, the calculated dynamic variations of the droplet profile with current pulses and dynamic development of the droplet tip vertical coordinate are shown in Figure 5.10. The longer time interval of $6 \mathrm{~ms}$, between the exciting pulse and the detaching pulse, allows the droplet to reach its highest position and move a long way down before the detaching pulse arrives. The downward momentum of the droplet is decreasing as the droplet moves its way down from its highest position. The joint effect of the decreasing downward momentum and the increasing electromagnetic force is not 
large enough to detach the droplet. Hence, phase match as a necessary condition for the proposed approach must be satisfied.

Phase match can be achieved by the proper selection of time interval $T_{b 2}$ when other parameters including the average current, pulse cycle frequency, peak current, base current and exciting pulse duration are kept constant. It becomes difficult to determine $T_{b 2}$ experimentally due to the use of the high-cost imaging system, but the diagnosis for phase match can be easily done numerically. When determining the value of $T_{b 2}$ to assure the phase match, the calculation is carried out first to simulate the response of the droplet under the stimulus of the exciting pulse.

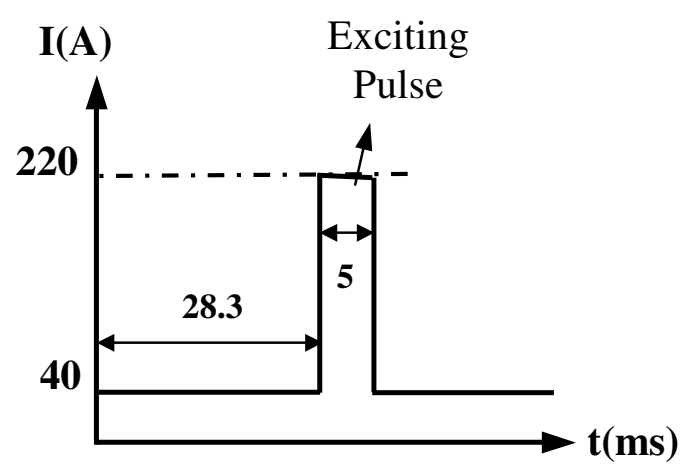

(a)

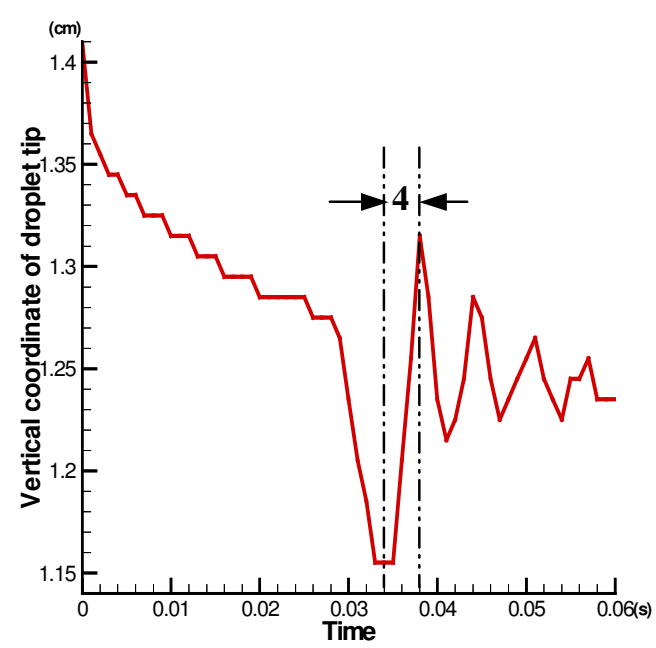

(b)

Figure 5.11 The droplet response to exciting pulse:

(a) Current waveform (b) Vertical coordinate of droplet tip 


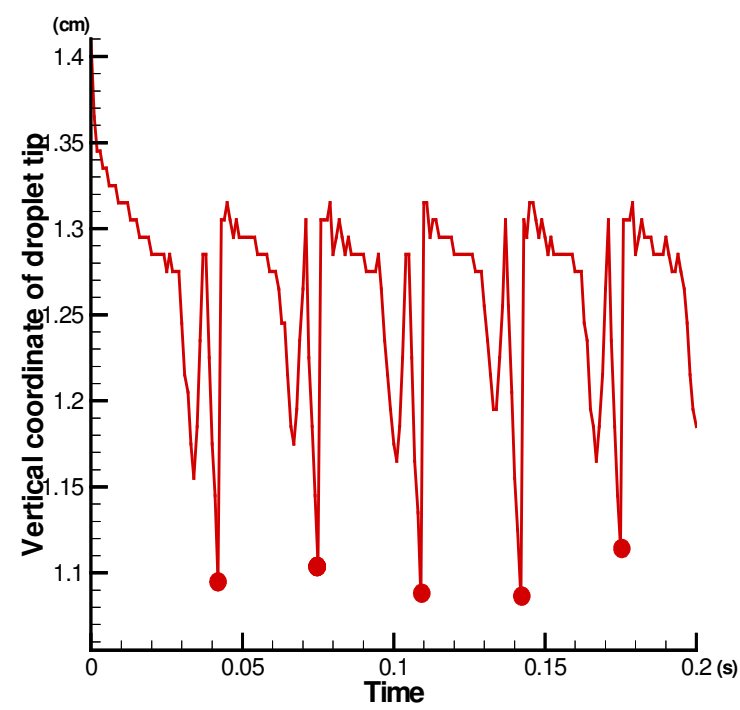

(a)

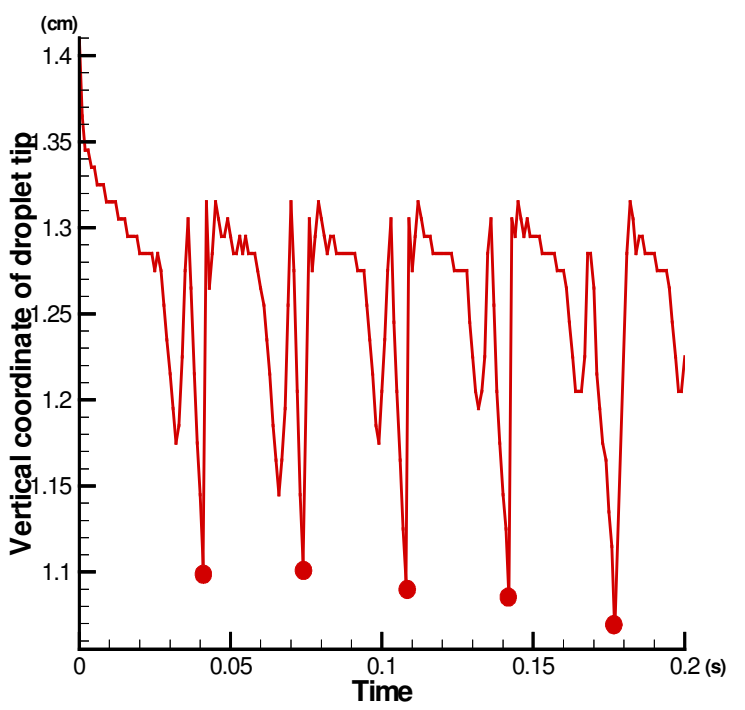

(c)

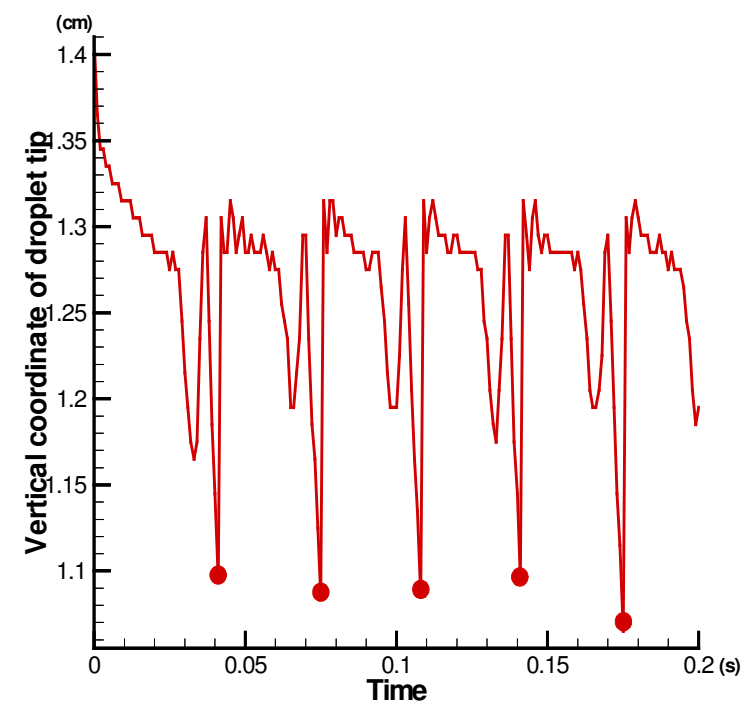

(b)

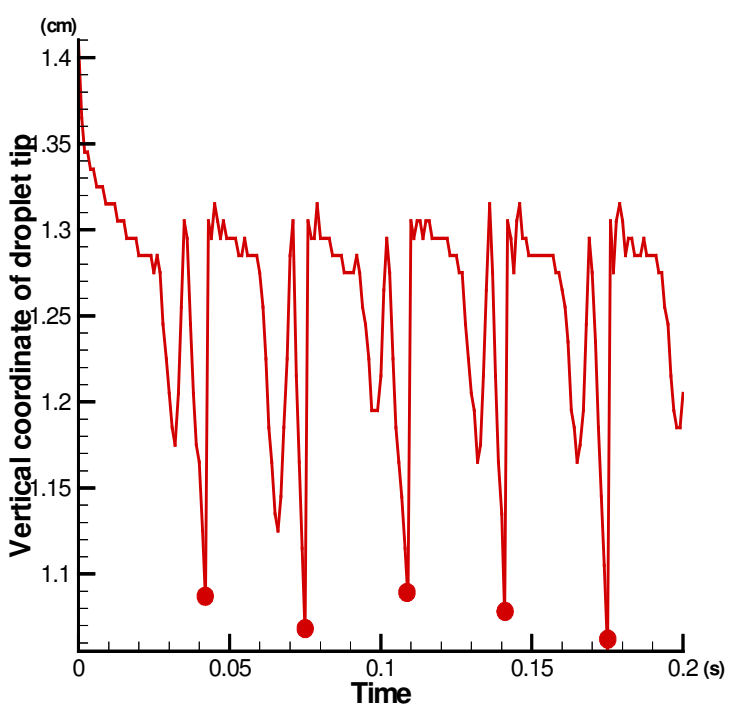

(d)

Figure 5.12 Development for vertical coordinate of droplet tip in modified pulsed current GMAW:
(a) ODPP under $\mathrm{T}_{\mathrm{b} 2}$ of $3.5 \mathrm{~ms}$
(b) ODPP under $\mathrm{T}_{\mathrm{b} 2}$ of $4.5 \mathrm{~ms}$
(c) ODPP under $\mathrm{T}_{\mathrm{b} 2}$ of $5 \mathrm{~ms}$
(d) ODPP under $\mathrm{T}_{\mathrm{b} 2}$ of $5.5 \mathrm{~ms}$ 
Figure 5.11 shows the calculated vertical coordinate development of the droplet tip under the application of the exciting pulse $I_{p 1}$. The oscillation of the droplet is induced by the sudden change of welding current. In order to realize the phase match and take advantage of the downward momentum of the oscillating droplet, it has been determined that the proper time to add the detaching pulse is when the vertical coordinate of the droplet tip reaches the highest point and it is ready to move downward. From Figure 5.11, this time is identified as $4 \mathrm{~ms}$ for the droplet recoiling back to its highest point after the exciting pulse under the given signal. According to previous calculation, ODPP metal transfer is realized when $T_{b 2}$ is set as $4 \mathrm{~ms}$.

Figure 5.12 shows metal transfers with ODPP are achieved for $T_{b 2}$ values between $3.5 \mathrm{~ms}$ and $5.5 \mathrm{~ms}$ when other parameters are hold constant. The instants for droplet detachment are marked with dots in the figure. Hence, the value of time interval $T_{b 2}$ which produces ODPP metal transfer can be determined according to analysis of the simulation results. It is noted that there is a time range around the instant of the droplet tip reaching the highest position, over which ODPP metal transfer could be obtained when the detaching pulse is added.

\subsubsection{The Operating Range of $T_{b 2}$}

The range of time interval $T_{b 2}$ which provides ODPP metal transfer when other parameters including the average current, pulse cycle frequency, peak current, base current and exciting pulse duration are kept constant is referred to as the operating range 
of $T_{b 2}$. From the above numerical calculation, the operating range of $T_{b 2}$ is predicted as $3.5 \mathrm{~ms}-5.7 \mathrm{~ms}$ when the average current $I_{\text {avg }}$ is $100 \mathrm{~A}$, the pulse cycle frequency $f$ is $30 \mathrm{~Hz}$, the base current $I_{b}$ is $40 \mathrm{~A}$, the peak current $I_{p}$ is $220 \mathrm{~A}$ and the exciting pulse duration $T_{p 1}$ is set as $5 \mathrm{~ms}$. In the modified pulsed current GMAW, the time interval $T_{b 2}$ is a critical parameter to obtain desirable ODPP metal transfer. Hence, the effect of other parameters on the operating range of $T_{b 2}$ are simulated and analyzed.

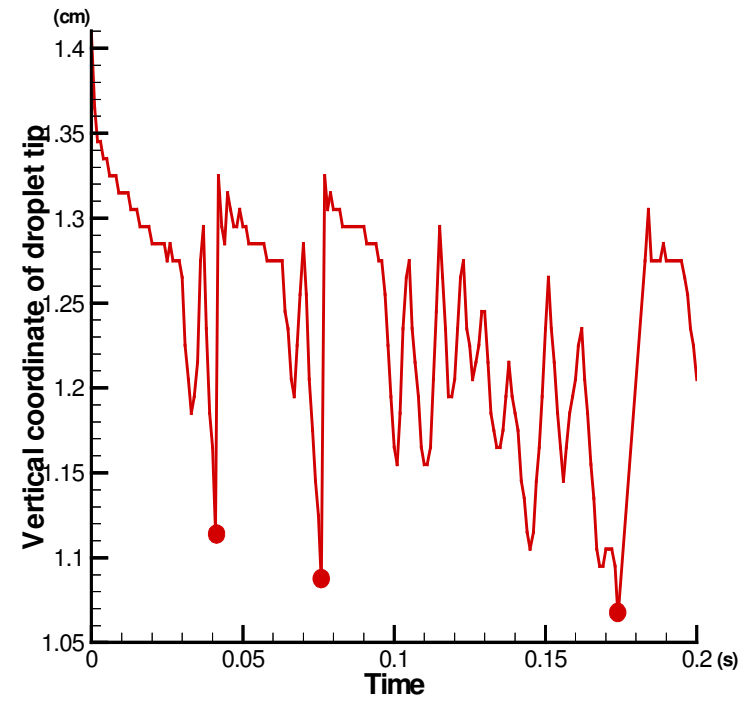

(a)

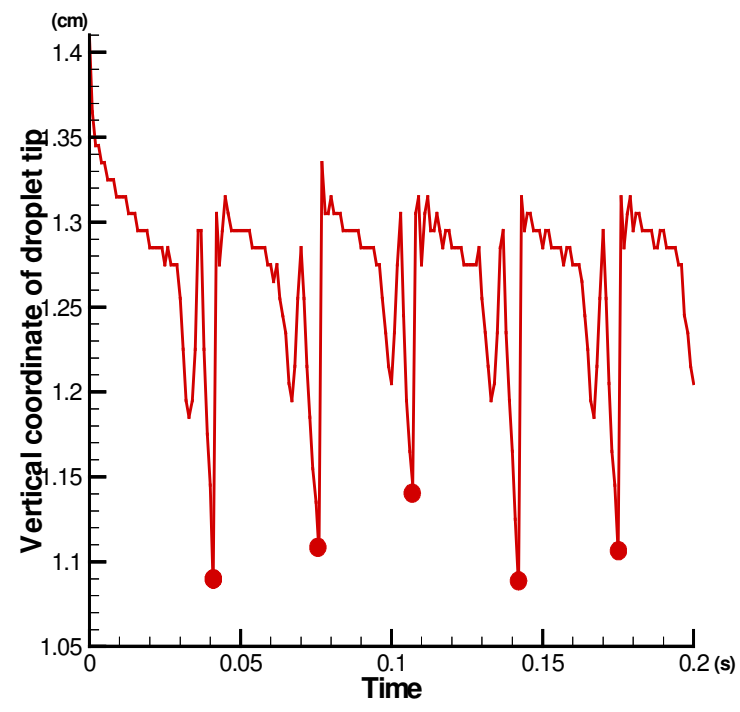

(b) 


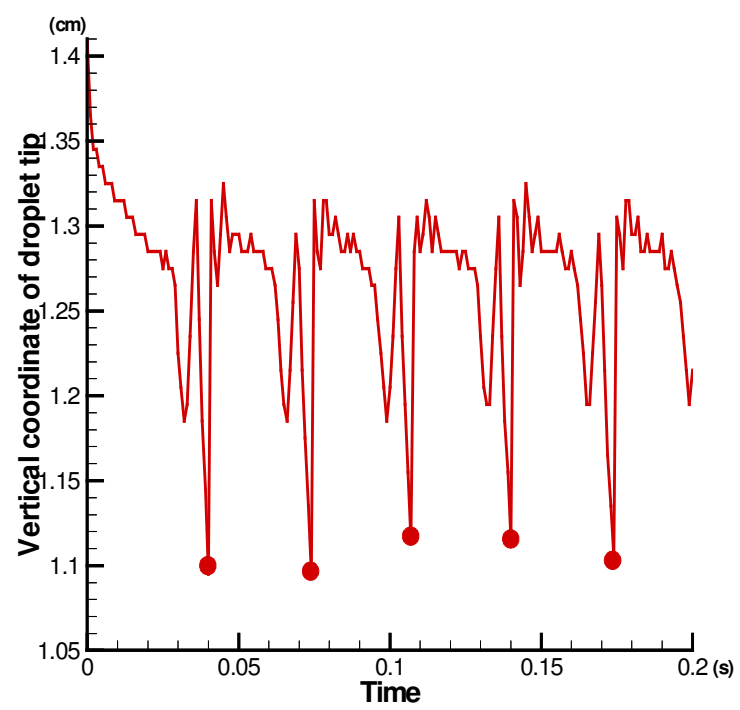

(c)

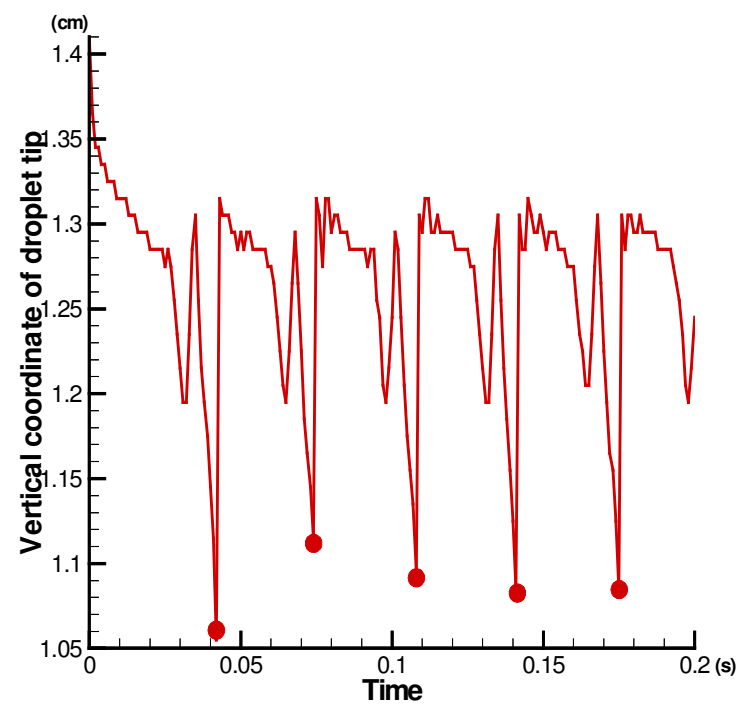

(e)

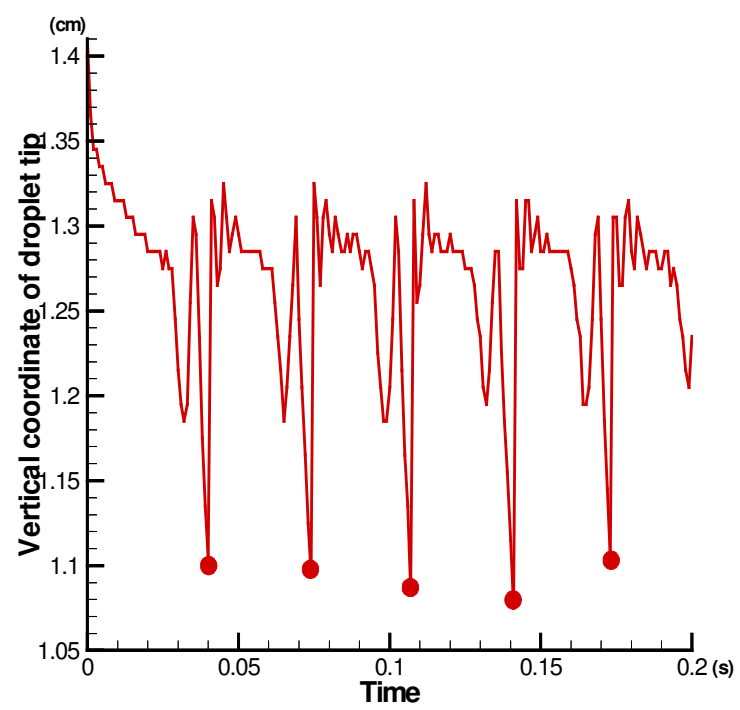

(d)

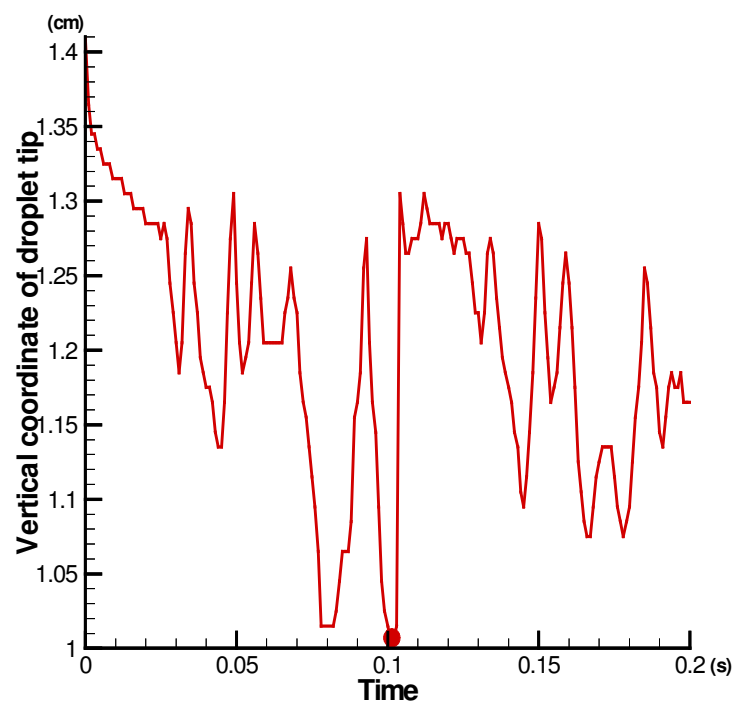

(f)

Figure 5.13 Development for vertical coordinate of droplet tip in modified pulsed current GMAW $\left(T_{p l}=4 \mathrm{~ms}, I_{a v g}=100 \mathrm{~A}, f=30 \mathrm{~Hz}, I_{p}=220 \mathrm{~A}, I_{b}=40 \mathrm{~A}\right)$ :
(a) ODMP under $\mathrm{T}_{\mathrm{b} 2}$ of $2.8 \mathrm{~ms}$
(b) ODPP under $\mathrm{T}_{\mathrm{b} 2}$ of $3 \mathrm{~ms}$
(c) ODPP under $\mathrm{T}_{\mathrm{b} 2}$ of $4 \mathrm{~ms}$
(d) ODPP under $\mathrm{T}_{\mathrm{b} 2}$ of $4.5 \mathrm{~ms}$
(e) ODPP under $\mathrm{T}_{\mathrm{b} 2}$ of $5 \mathrm{~ms}$
(f) ODMP under $\mathrm{T}_{\mathrm{b} 2}$ of $5.5 \mathrm{~ms}$ 


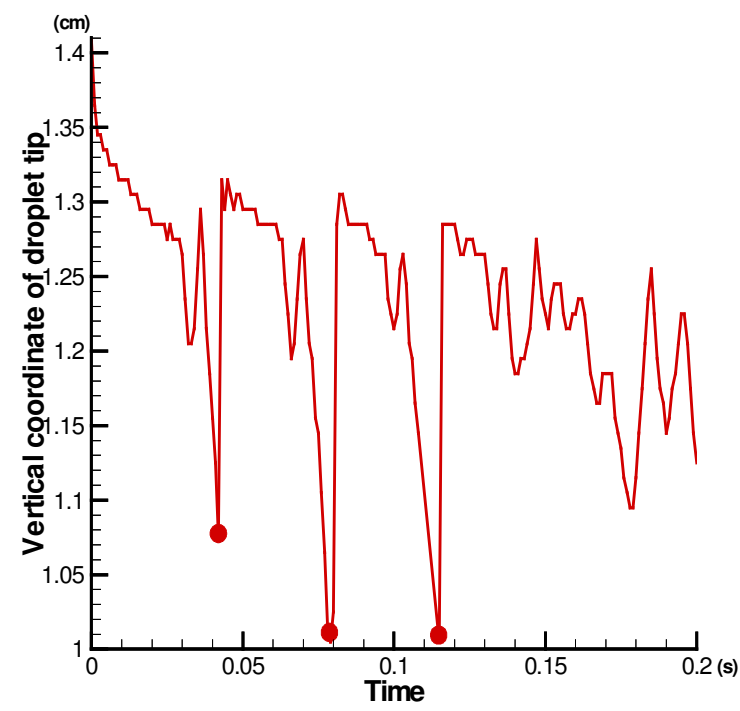

(a)

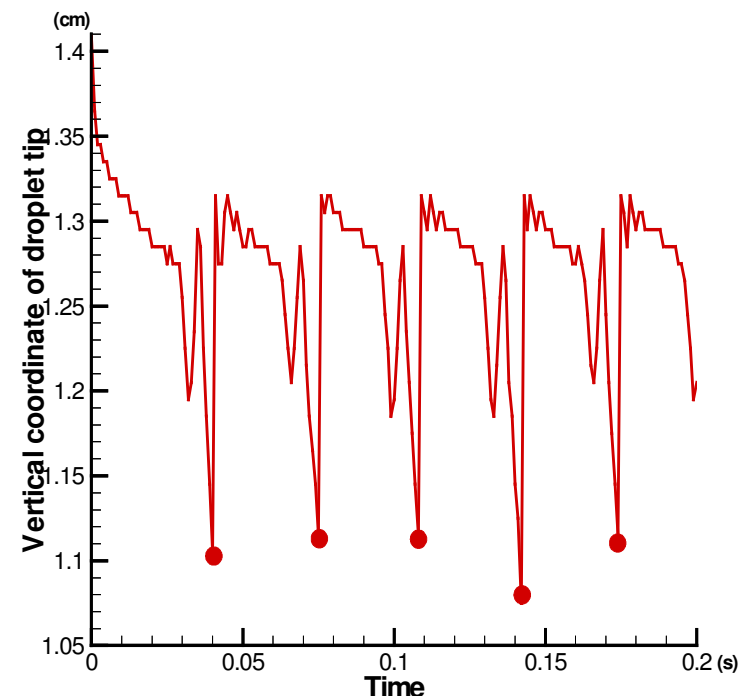

(c)

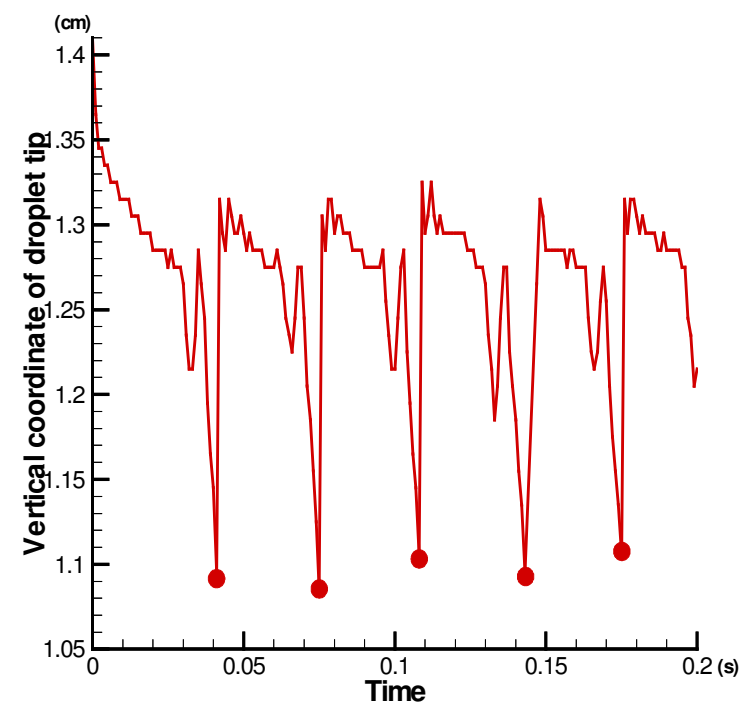

(b)

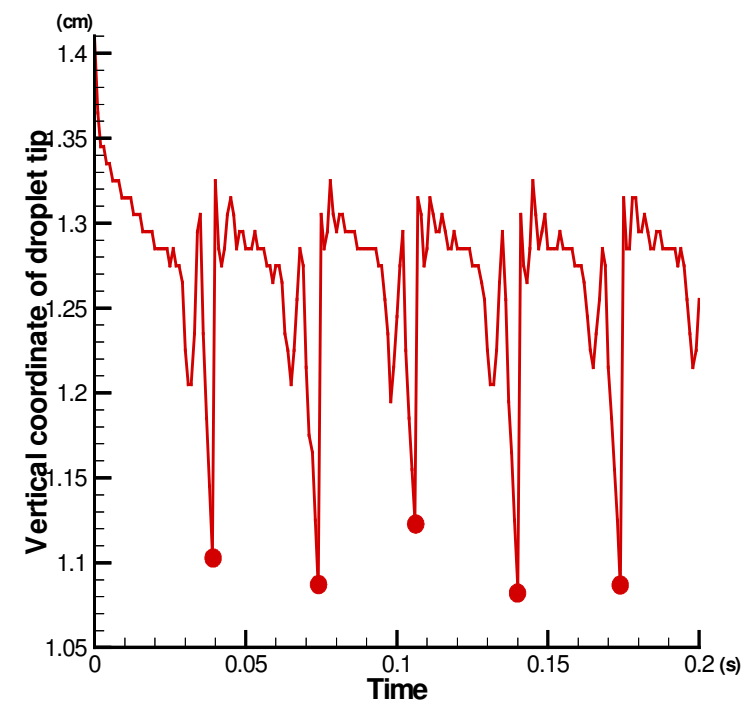

(d) 


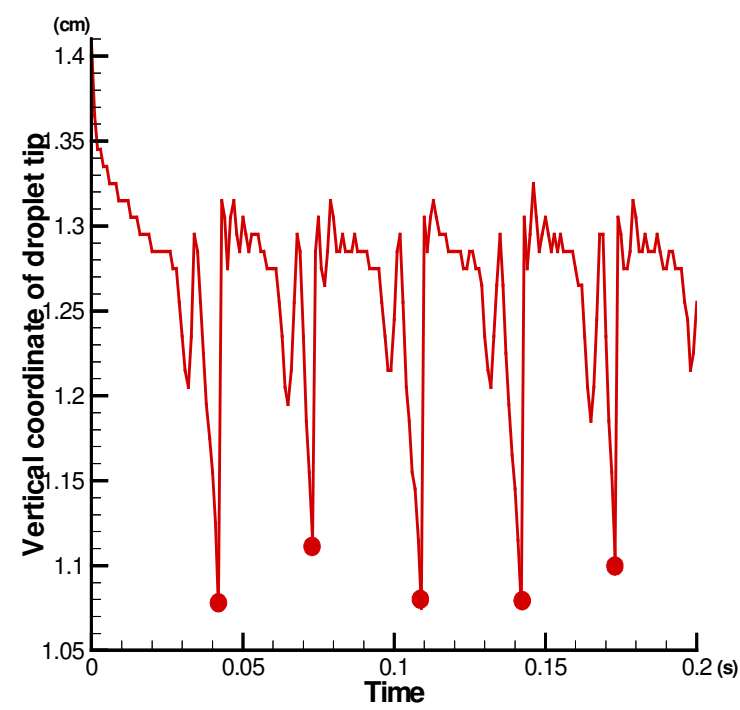

(e)

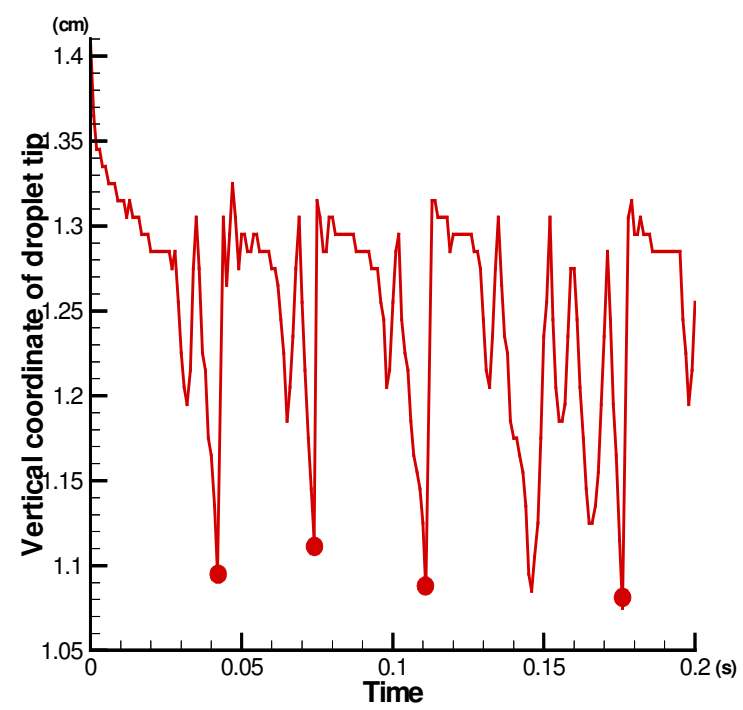

(f)

Figure 5.14 Development for vertical coordinate of droplet tip in modified pulsed current GMAW $\left(T_{p l}=3 \mathrm{~ms}, I_{a v g}=100 \mathrm{~A}, f=30 \mathrm{~Hz}, I_{p}=220 \mathrm{~A}, I_{b}=40 \mathrm{~A}\right)$ :
(a) ODMP under $T_{b 2}$ of $2.5 \mathrm{~ms}$
(b) ODPP under $\mathrm{T}_{\mathrm{b} 2}$ of $2.8 \mathrm{~ms}$
(c) ODPP under $\mathrm{T}_{\mathrm{b} 2}$ of $3 \mathrm{~ms}$
(d) ODPP under $\mathrm{T}_{\mathrm{b} 2}$ of $4 \mathrm{~ms}$
(e) ODPP under $\mathrm{T}_{\mathrm{b} 2}$ of $5 \mathrm{~ms}$
(f) ODMP under $T_{b 2}$ of $5.5 \mathrm{~ms}$

When the exciting pulse duration $T_{p 1}$ is decreased to $4 \mathrm{~ms}$ with the same average current, pulse cycle frequency, base current and peak current, the calculated dynamic developments of droplet tip vertical coordinate for different values of $T_{b 2}$ are illustrated in Figure 5.13. Metal transfers with ODPP are achieved between $T_{b 2}$ of $3 \mathrm{~ms}$ and $T_{b 2}$ of $5.2 \mathrm{~ms}$. In other words, the operating range of $T_{b 2}$ is predicted to be between $3 \mathrm{~ms}$ and 5.2ms. As the exciting pulse duration $T_{p 1}$ is further decreased to $3 \mathrm{~ms}$, the predicted operating range of $T_{b 2}$ is $2.8 \mathrm{~ms}-5 \mathrm{~ms}$ as shown in Figure 5.14. 
It is noted that the operating range of $T_{b 2}$ is shifted slightly to a smaller value without much change in the interval as the exciting pulse duration $T_{p 1}$ is decreased from $5 \mathrm{~ms}$ to $3 \mathrm{~ms}$. The decrease of $T_{p 1}$ causes the mass of oscillating droplet to also decrease slightly. Less time is need for a smaller oscillating droplet bouncing to its highest position since the frequency of droplet oscillation after a current pulse increases with a decrease of droplet mass. Hence, the slight shift of the operating range of $T_{b 2}$ can be explained by a slight change of oscillating droplet mass due to the change of $T_{p 1}$. The same span of operating $T_{b 2}$ under the certain range of exciting pulse duration $T_{p 1}$ suggests that the exciting pulse with same peak current provides the same amount of momentum of droplet oscillation during that range of $T_{p 1}$. In other words, there is an operating range of $T_{p 1}$ which provides ODPP metal transfer with the proper selection of $T_{b 2}$.

When the exciting pulse duration $T_{p 1}$ is increased to $6 \mathrm{~ms}$, the calculated dynamic developments of droplet tip vertical coordinate for different values of $T_{b 2}$ are illustrated in Figure 5.15. The results show that the operating range of $T_{b 2}$ narrowed down suddenly to $4.5 \mathrm{~ms}-5 \mathrm{~ms}$. As indicated in Figure 5.16, ODPP metal transfer can no longer be achieved when $T_{p 1}$ is increased further to $7 \mathrm{~ms}$. This is because the decreasing duration of detaching pulse $T_{p 2}$ becomes too short to detach a droplet, when the duration of the exciting pulse $T_{p 1}$ rises above a certain value. 


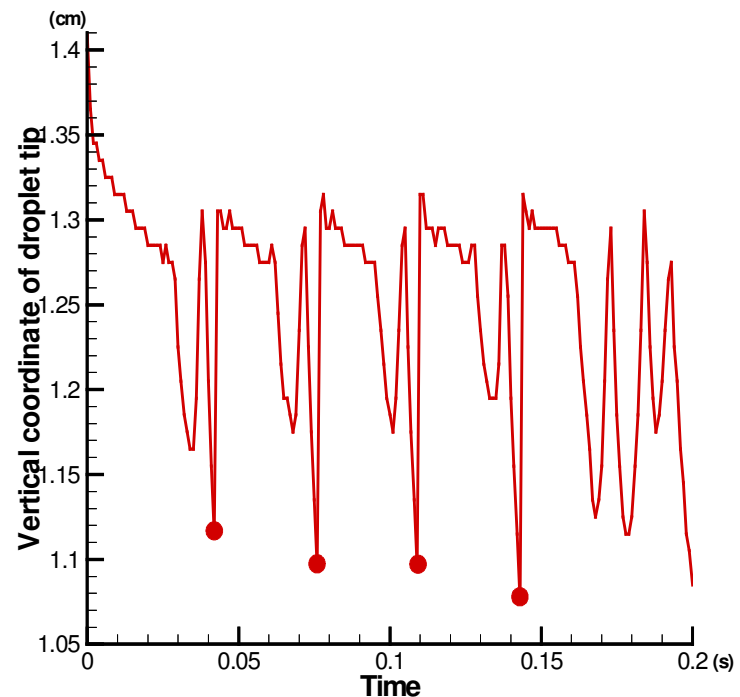

(a)

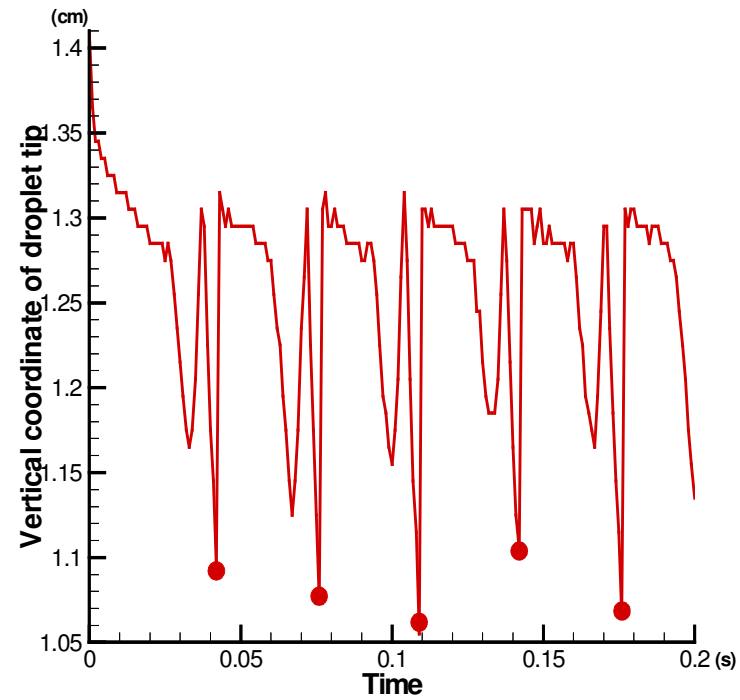

(c)

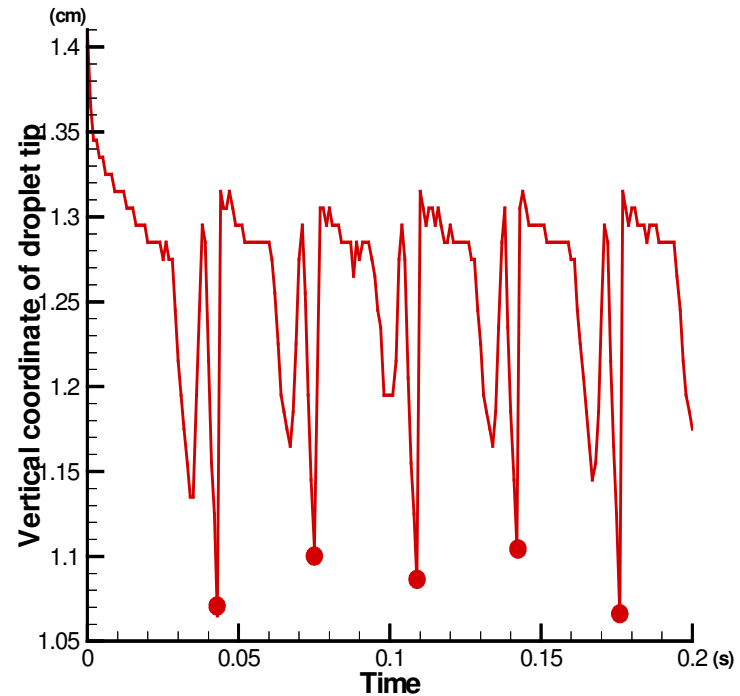

(b)

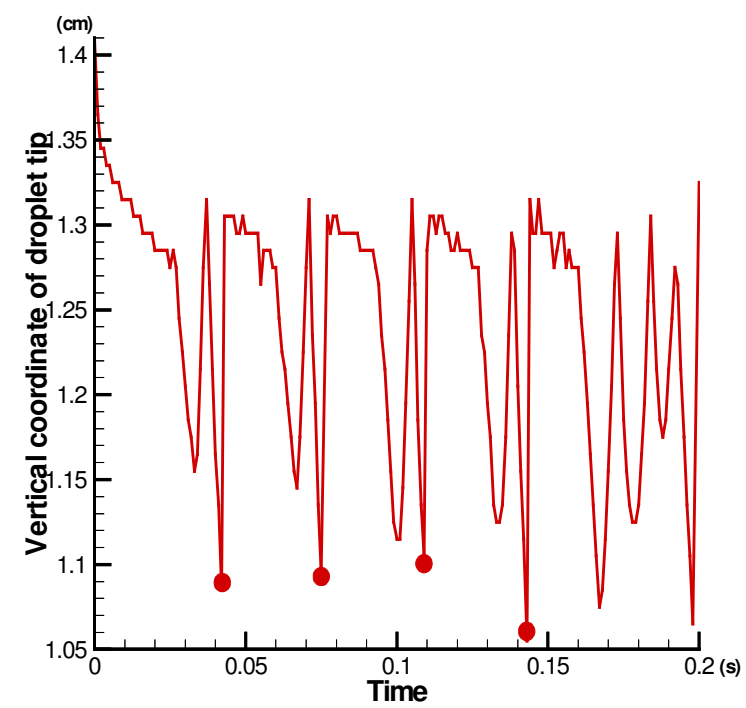

(d)

Figure 5.15 Development for vertical coordinate of droplet tip in modified pulsed current GMAW $\left(T_{p l}=6 \mathrm{~ms}, I_{a v g}=100 \mathrm{~A}, f=30 \mathrm{~Hz}, I_{p}=220 \mathrm{~A}, I_{b}=40 \mathrm{~A}\right)$ :
(a) ODMP under $\mathrm{T}_{\mathrm{b} 2}$ of $4 \mathrm{~ms}$
(b) ODPP under $\mathrm{T}_{\mathrm{b} 2}$ of $4.5 \mathrm{~ms}$
(c) ODPP under $\mathrm{T}_{\mathrm{b} 2}$ of $5 \mathrm{~ms}$
(d) ODMP under $\mathrm{T}_{\mathrm{b} 2}$ of $5.5 \mathrm{~ms}$ 


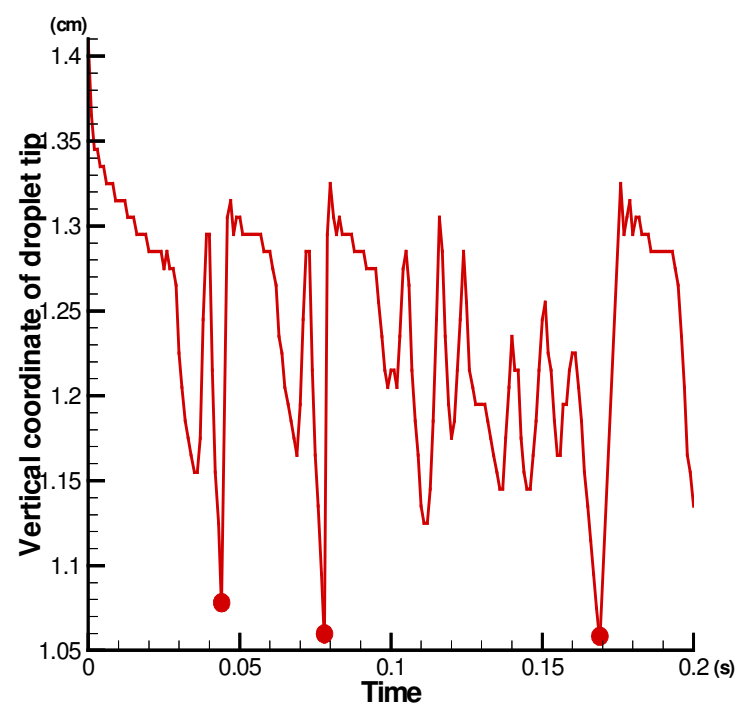

(a)

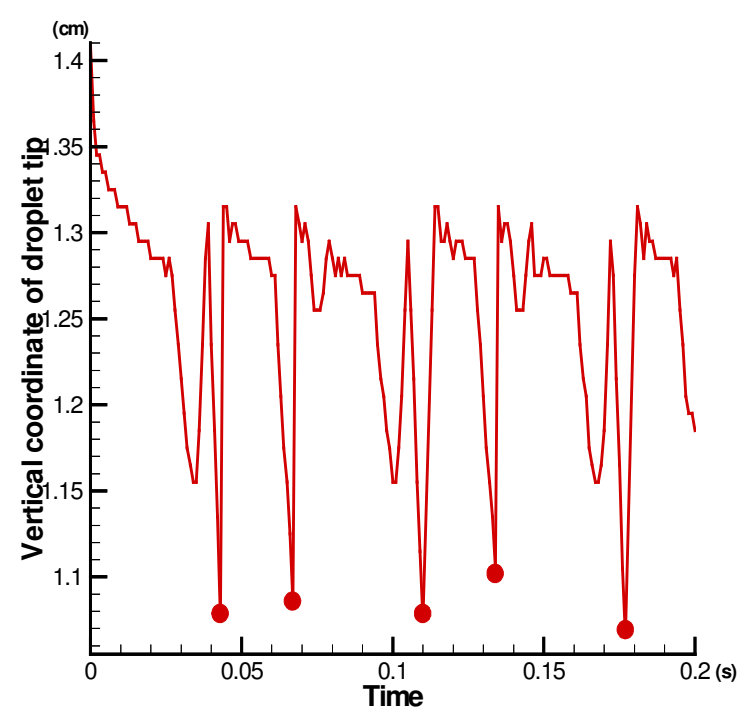

(b)

Figure 5.16 Development for vertical coordinate of droplet tip in modified pulsed current GMAW $\left(T_{p l}=7 \mathrm{~ms}, I_{a v g}=100 \mathrm{~A}, f=30 \mathrm{~Hz}, I_{p}=220 \mathrm{~A}, I_{b}=40 \mathrm{~A}\right)$ :

(a) ODMP under $\mathrm{T}_{\mathrm{b} 2}$ of $4 \mathrm{~ms}$ (b) ODMP under $\mathrm{T}_{\mathrm{b} 2}$ of $5 \mathrm{~ms}$

\subsubsection{The Influence of Pulsing Cycle Frequency and Peak Current}

The pulse frequency $f$ is increased to $40 \mathrm{~Hz}$ with other parameters including the average current (100A), peak current (220A) and base current (40A) are kept the same as the model case. The calculated droplet profiles and dynamic development of the droplet tip vertical coordinate under the given current signal are shown in Figure 5.17. Metal transfer with one droplet detached per pulse cycle can also be achieved. The calculated operating range of $T_{b 2}$ is $3 \mathrm{~ms}-3.5 \mathrm{~ms}$ as shown in Figure 5.18 . 


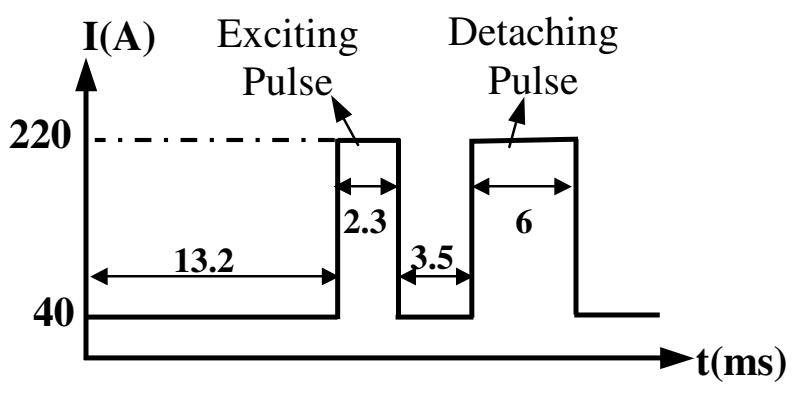

(a)
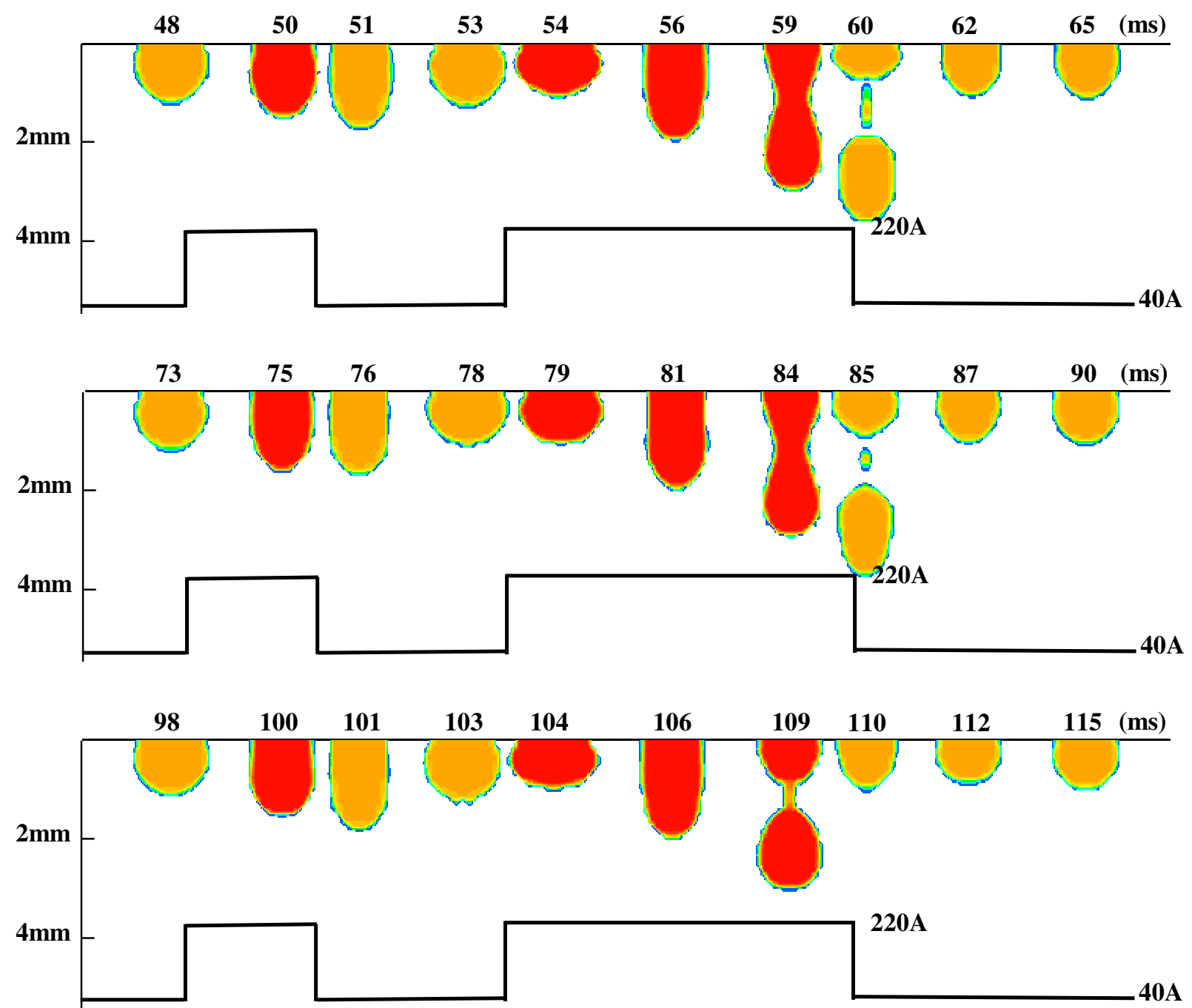

(b) 


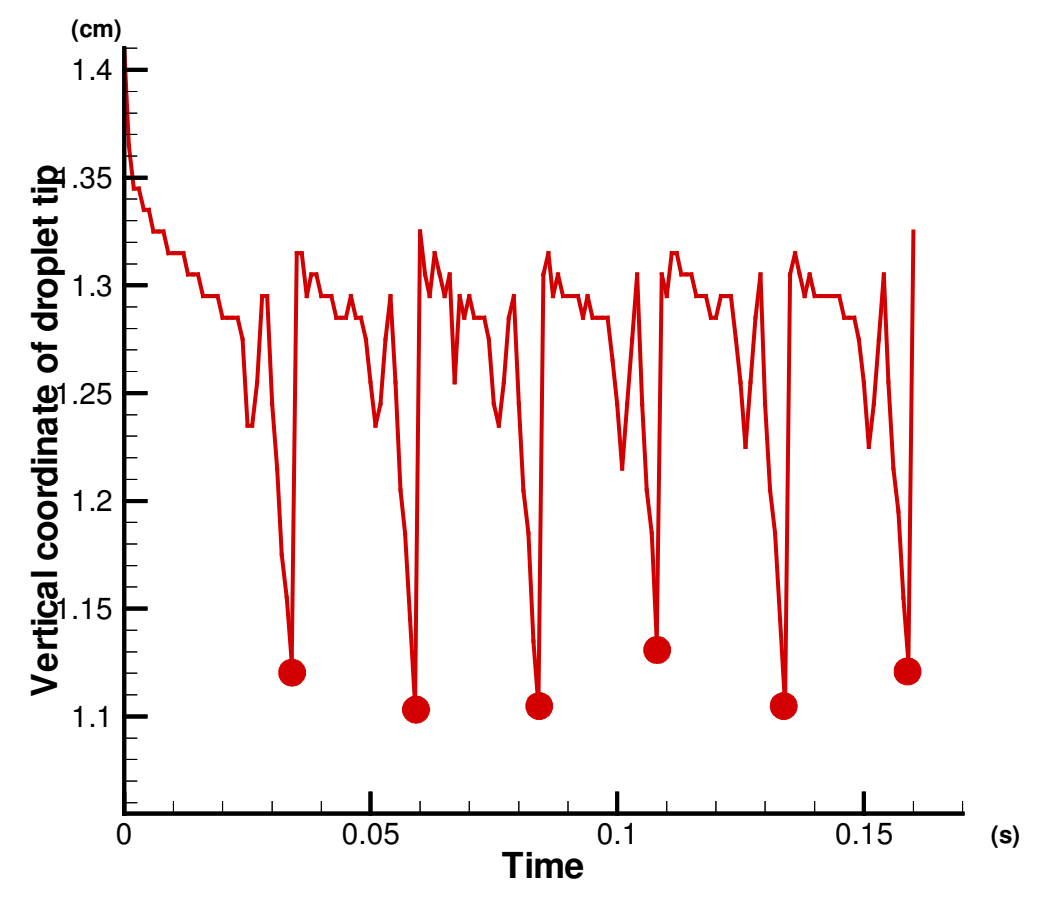

(c)

Figure 5.17 Metal transfer with ODPP under modified pulsed current GMAW:

(a) Current Signal (b) Droplet profiles (c) Vertical coordinate of droplet tip

The peak current $I_{p}$ is decreased to $210 \mathrm{~A}$ while other parameters including the average current $(100 \mathrm{~A})$, the pulse cycle frequency $(30 \mathrm{~Hz})$ and base current $(40 \mathrm{~A})$ are kept the same as the model case. The calculated droplet profiles and dynamic development of the droplet tip vertical coordinate under the given current signal are shown in Figure 5.19. Metal transfer with one drop detached per pulse cycle is predicted by using modified pulsed current GMAW. Figure 5.20 shows the calculated vertical coordinate development for the different choices of $T_{b 2}$. The operating range of $T_{b 2}$ is identified to be from $3 \mathrm{~ms}$ to $4 \mathrm{~ms}$. 


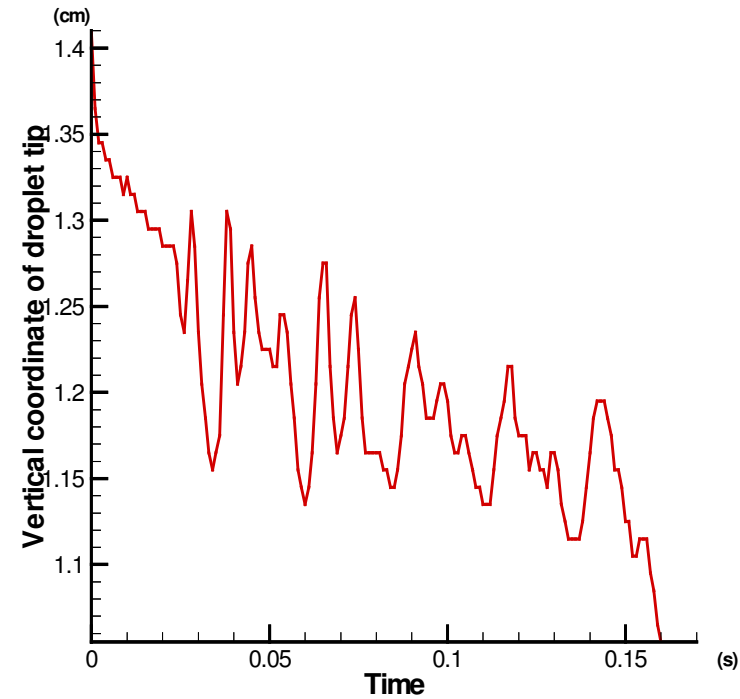

(a)

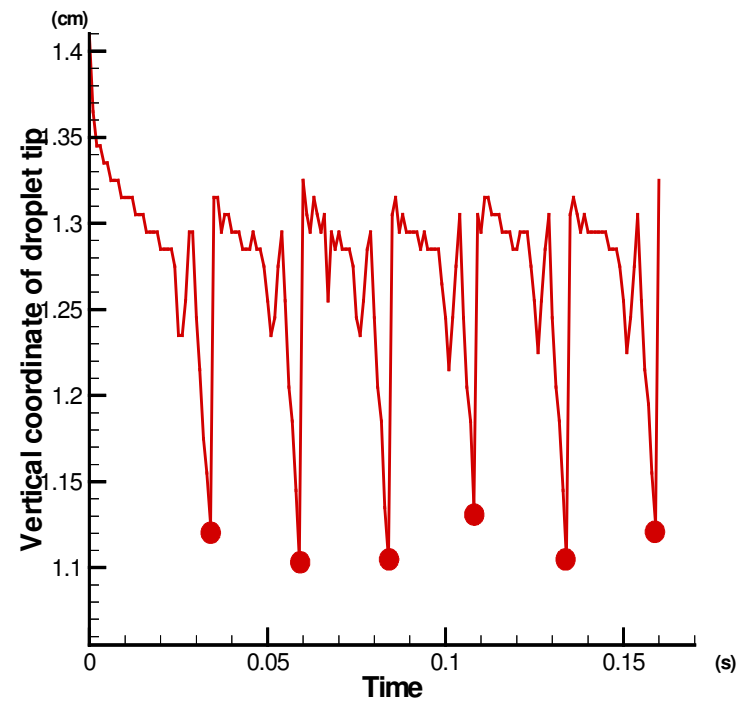

(c)

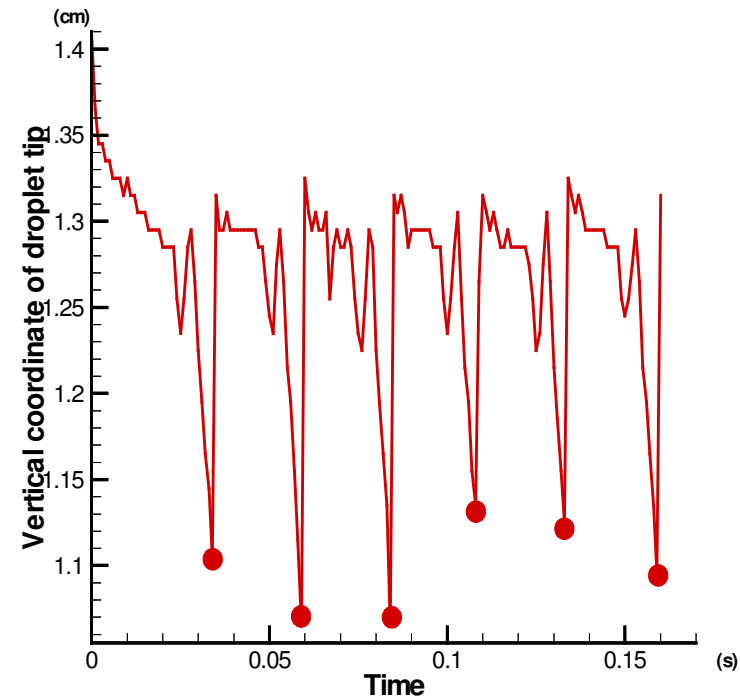

(b)

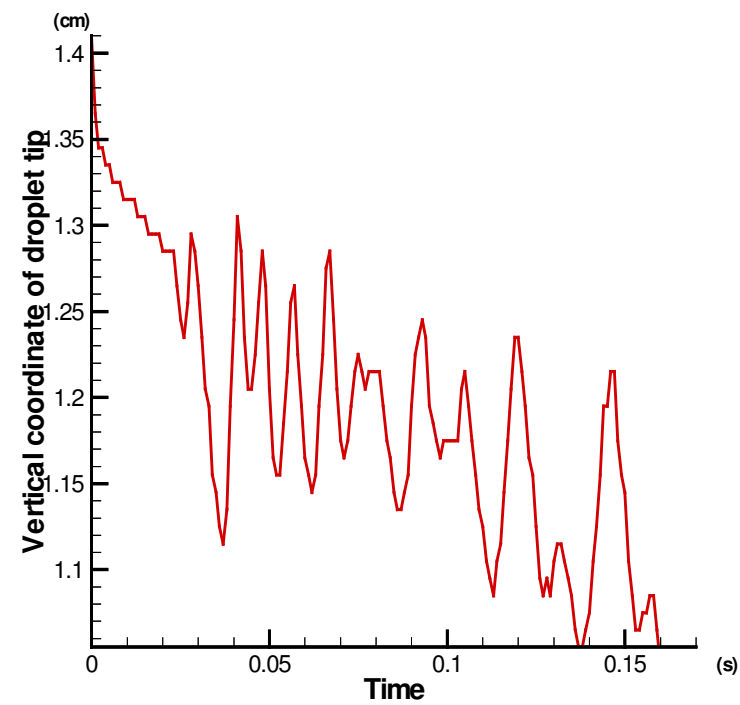

(d)

Figure 5.18 Development for vertical coordinate of droplet tip in modified pulsed current GMAW:

(a) ODMP under $\mathrm{T}_{\mathrm{b} 2}$ of $2.5 \mathrm{~ms}$ (b) ODPP under $\mathrm{T}_{\mathrm{b} 2}$ of $3 \mathrm{~ms}$

$\begin{array}{ll}\text { (c) ODPP under } \mathrm{T}_{\mathrm{b} 2} \text { of } 3.5 \mathrm{~ms} & \text { (d) ODMP under } \mathrm{T}_{\mathrm{b} 2} \text { of } 4 \mathrm{~ms}\end{array}$ 

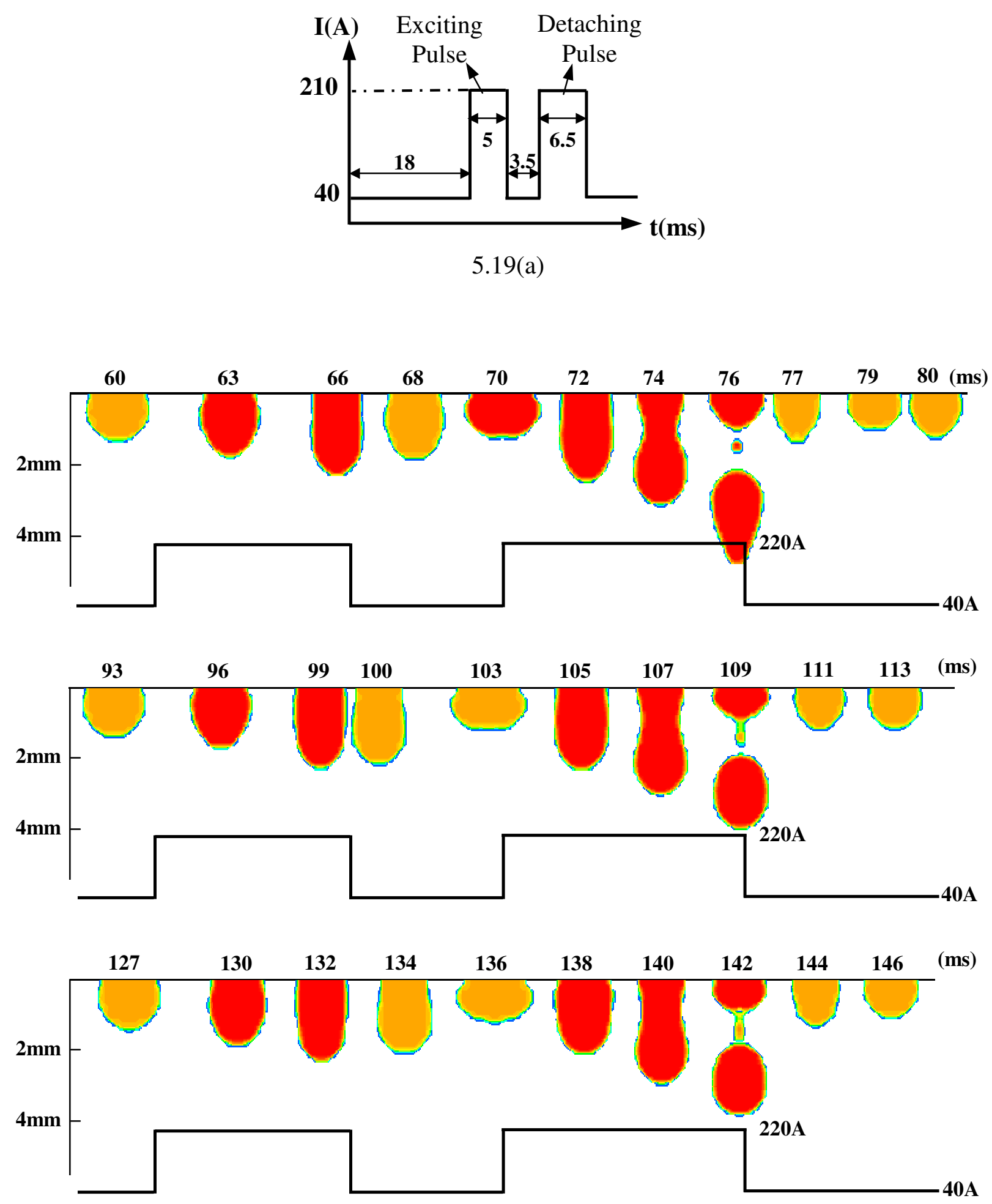

5.19 (b) 


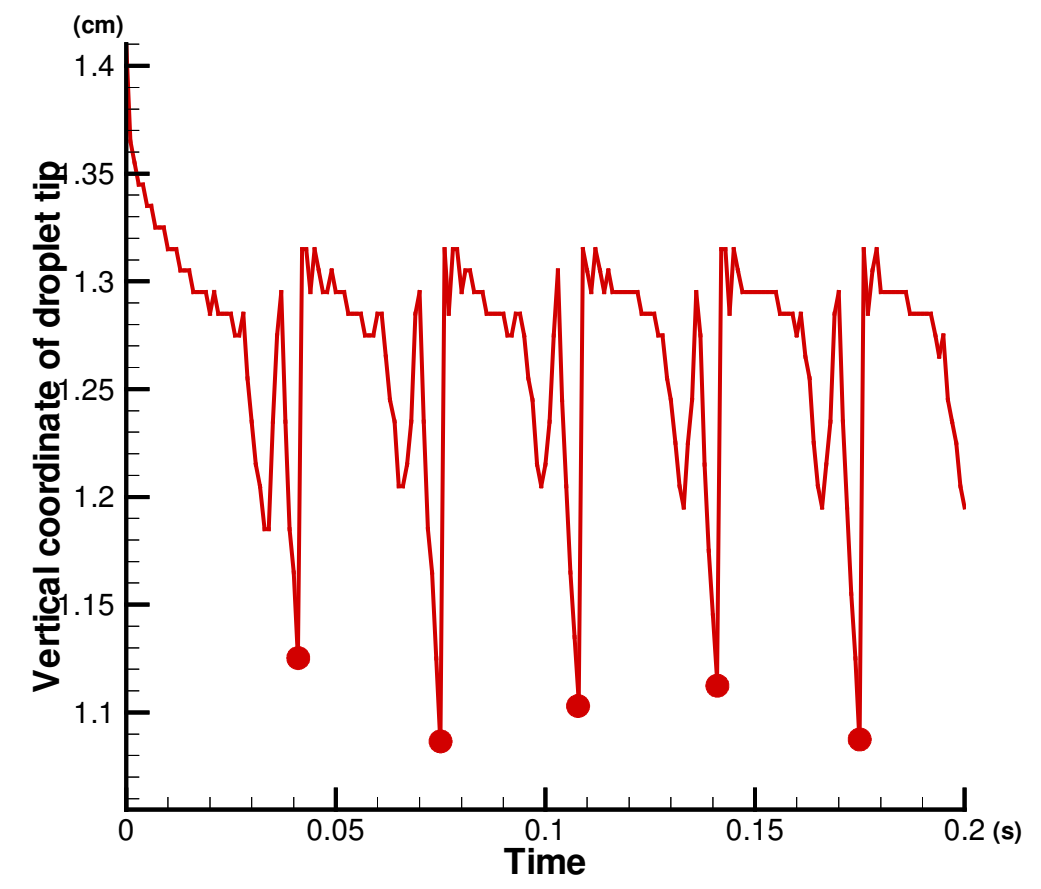

$5.19(\mathrm{c})$

Figure 5.19 Metal transfer with ODPP under modified pulsed current GMAW:

(a) Current waveform (b) Droplet profiles (c) Vertical coordinate of droplet tip

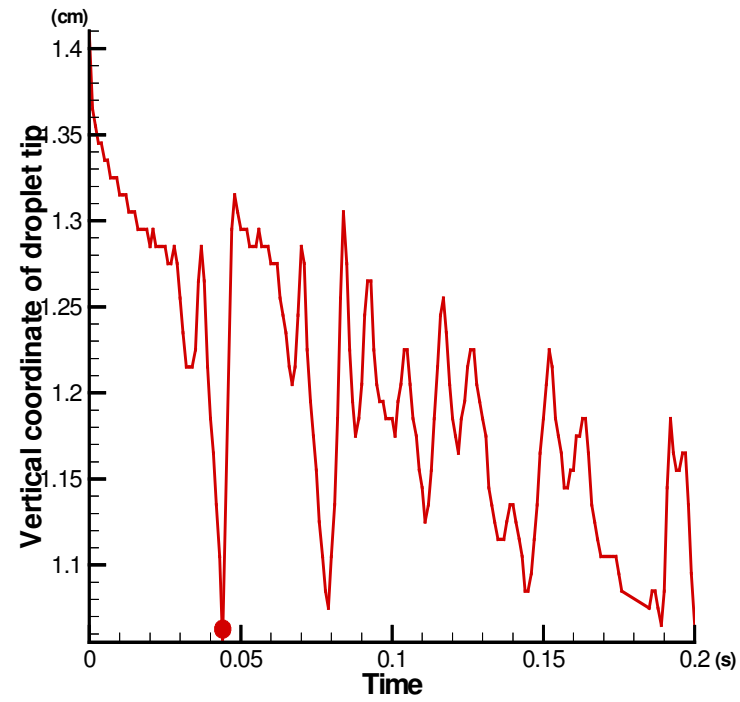

(a)

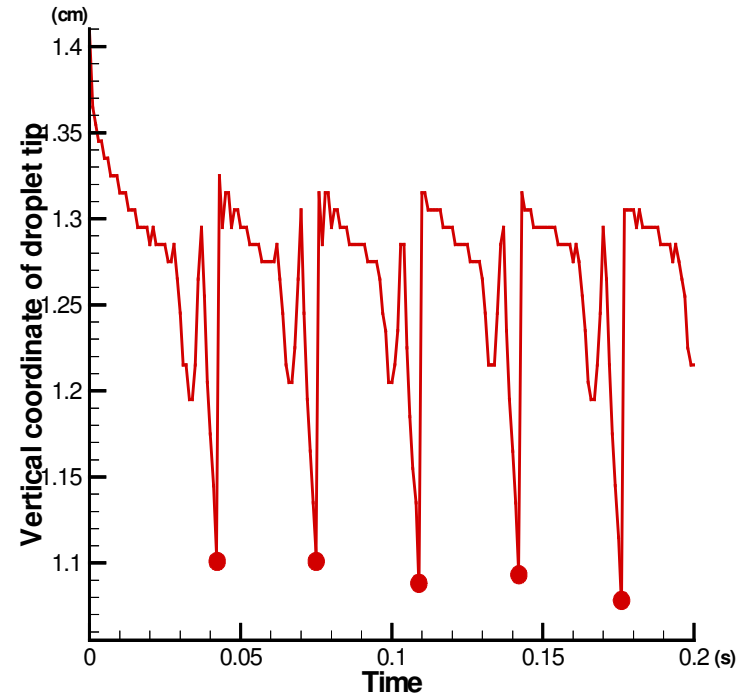

(b) 


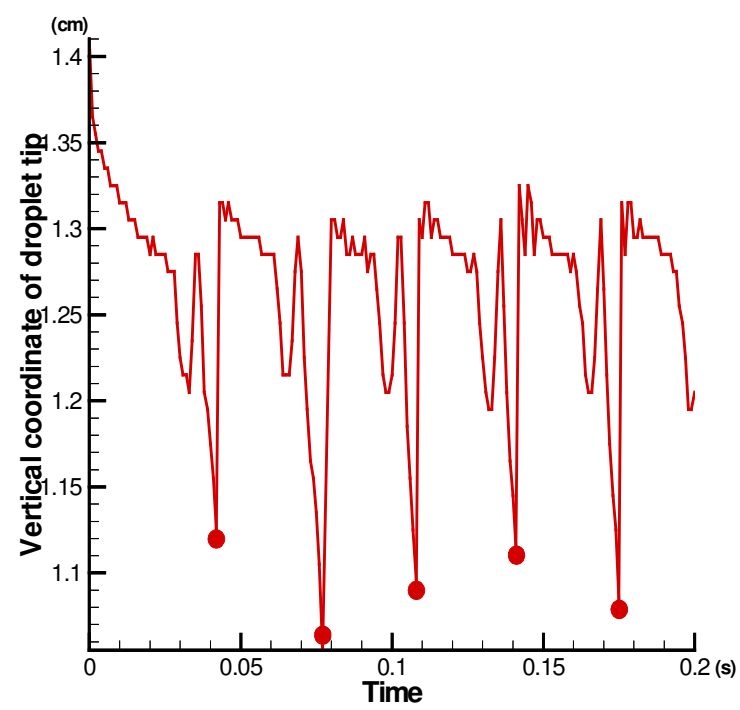

(c)

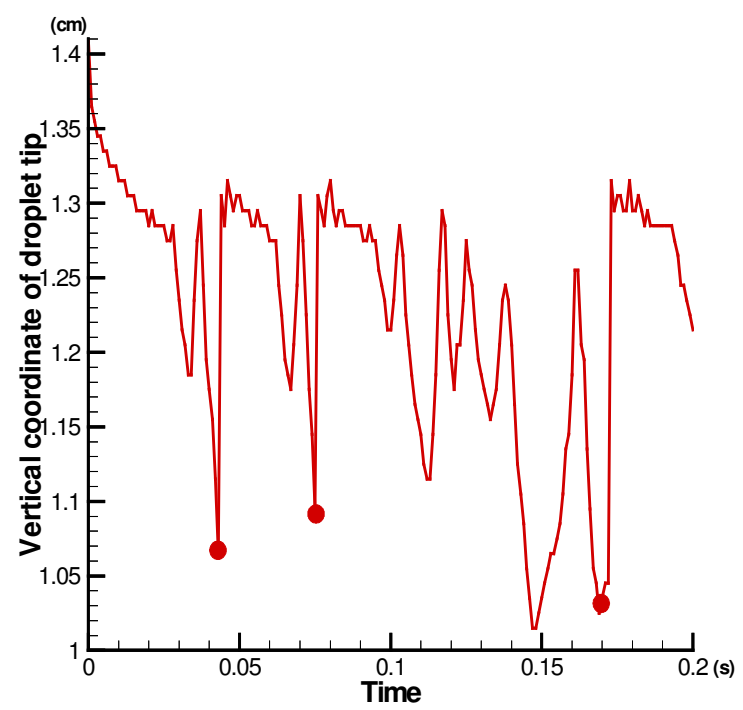

(d)

Figure 5.20 Development for vertical coordinate of droplet tip in modified pulsed current GMAW:
(a) ODMP under $\mathrm{T}_{\mathrm{b} 2}$ of $2.5 \mathrm{~ms}$
(b) ODPP under $\mathrm{T}_{\mathrm{b} 2}$ of $3 \mathrm{~ms}$
(c) ODPP under $\mathrm{T}_{\mathrm{b} 2}$ of $4 \mathrm{~ms}$
(d) ODMP under $\mathrm{T}_{\mathrm{b} 2}$ of $4.5 \mathrm{~ms}$

Narrower operating ranges of $T_{b 2}$ are obtained as the pulse cycle frequency increases or the peak current decreases. A theoretical explanation for this phenomenon can easily be found. When other parameters stay unchanged, an increase in pulse cycle frequency produces a smaller droplet if ODPP metal transfer occurs. A larger combination of droplet downward momentum and electromagnetic force is required to detach a smaller droplet, and therefore brings down the operating range of $T_{b 2}$. The decrease of peak current causes the decrease of both electromagnetic force and downward momentum of droplet oscillation, and therefore narrows the operating range of $T_{b 2}$. 
The same value of peak current for the exciting pulse and the detaching pulse has been used in previous cases. The peak current has been kept lower than the transition current to prevent accidental droplet detachment, and also to keep thin-sectioned or heat sensitive workpieces from overheating. In the following cases, different values of peak current are used for the exciting pulse and detaching pulse. The peak current for the exciting pulse is still kept under the transition current to prevent accidental detachment, while the peak current for the detaching pulse becomes adjustable.

The exciting pulse still provides downward momentum of droplet oscillation to enhance detachment, and at the same time prevents accidental droplet detachment. A higher current could be used as the peak current for the detaching pulse. The increase of detaching pulse peak current reinforces the electromagnetic force produced by the detaching pulse, and therefore provides strengthened detachment force and assists the droplet detachment.

The peak current for the detaching pulse is increased to $250 \mathrm{~A}$ while the other parameters including average current (100A), pulse cycle frequency (30A), base current (40A), peak current (220A) and duration (5ms) for the exciting pulse are kept the same as the model case. Figure 5.21 illustrates the calculated dynamic developments of droplet tip vertical coordinate for different choices of time interval $T_{b 2}$. Metal transfers with ODPP are achieved between $T_{b 2}$ of $3 \mathrm{~ms}$ and $T_{b 2}$ of $6 \mathrm{~ms}$. In comparison with the model case (3.5ms-5.7ms), the operating range of $T_{b 2}$ has been expanded. 


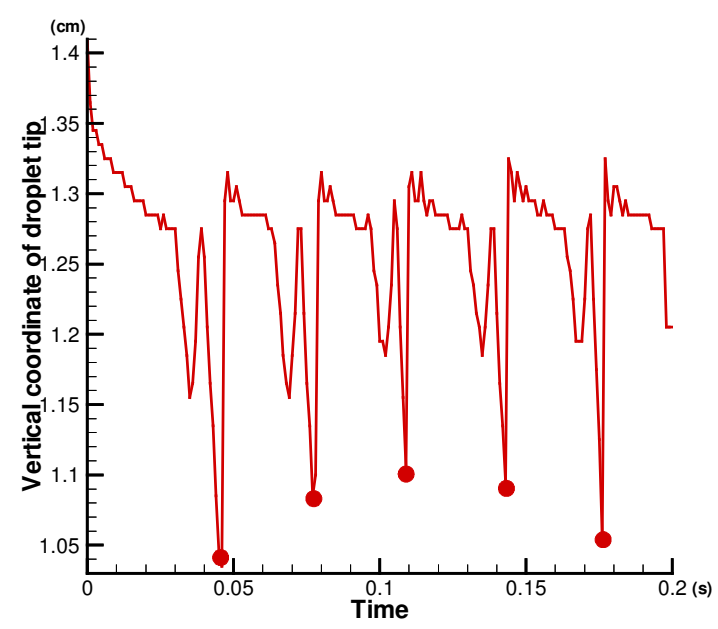

(a)

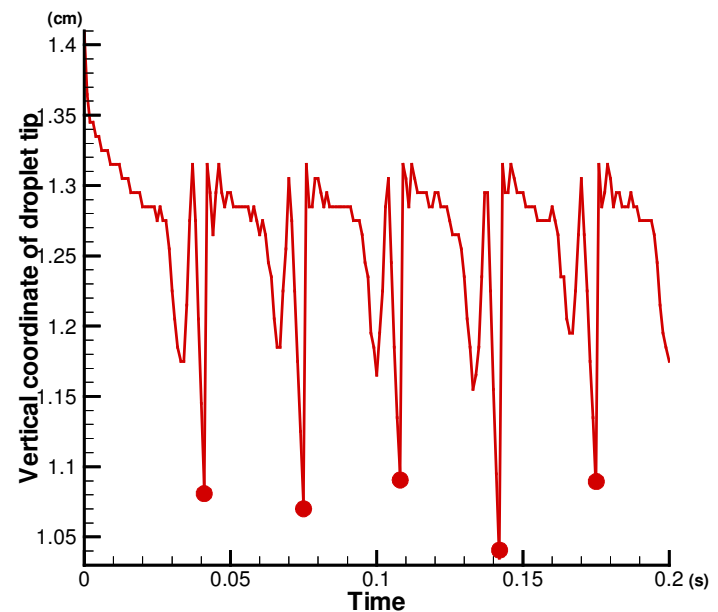

(c)

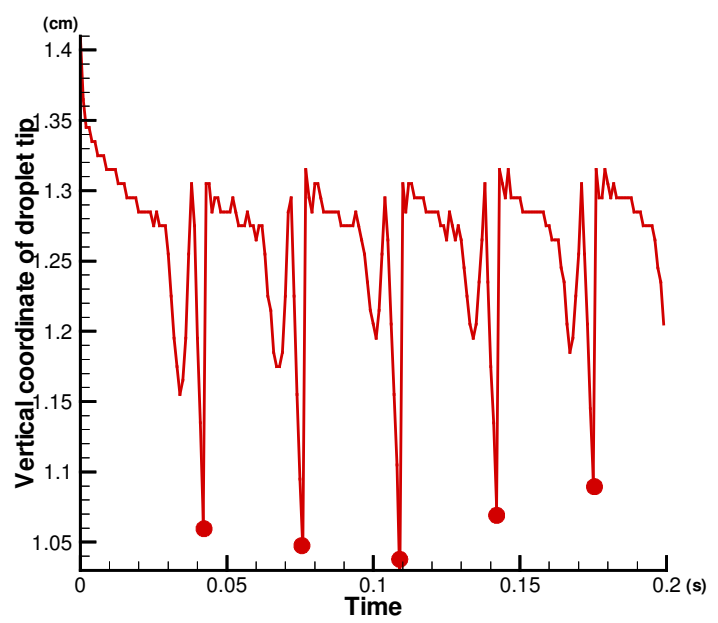

(b)

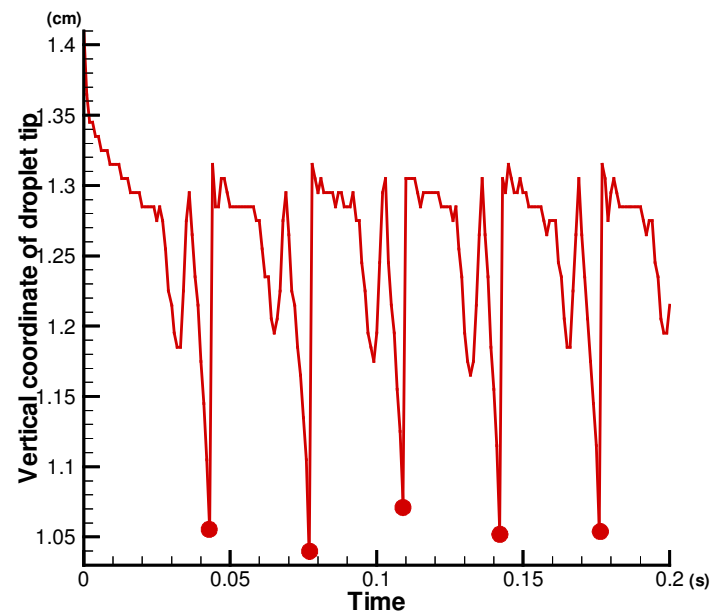

(d)

Figure 5.21 Development for vertical coordinate of droplet tip in modified pulsed current GMAW ( $I_{p l}=220, I_{p 2}=250$ ):
(a) ODPP under $\mathrm{T}_{\mathrm{b} 2}$ of $3 \mathrm{~ms}$
(b) ODPP under $\mathrm{T}_{\mathrm{b} 2}$ of $4 \mathrm{~ms}$
(c) ODPP under $\mathrm{T}_{\mathrm{b} 2}$ of $5 \mathrm{~ms}$
(d) ODPP under $\mathrm{T}_{\mathrm{b} 2}$ of $6 \mathrm{~ms}$ 
The peak current for the detaching pulse is further increased to 300A. As we already known, the undesirable streaming spray metal transfer with high-speed small droplets occurs under constant current of 300A in GMAW. When the average current $(100 \mathrm{~A})$, pulse cycle frequency $(30 \mathrm{~Hz})$ and base current $(40 \mathrm{~A})$ are set the same as the model case, the metal transfer under conventional single-pulsed-current GMAW with peak current of $300 \mathrm{~A}$ is also considered for the purpose of comparison with modified pulsed current GMAW. Calculation is carried out first for metal transfer under the conventional single-pulsed-current GMAW. Figure 5.22 shows the calculated developments for vertical coordinate of droplet tip and droplet profiles. Multiple droplets are detached per pulse, i.e., accidental droplet detachment occurs in the conventional pulsed current GMAW under the above assumed welding conditions.

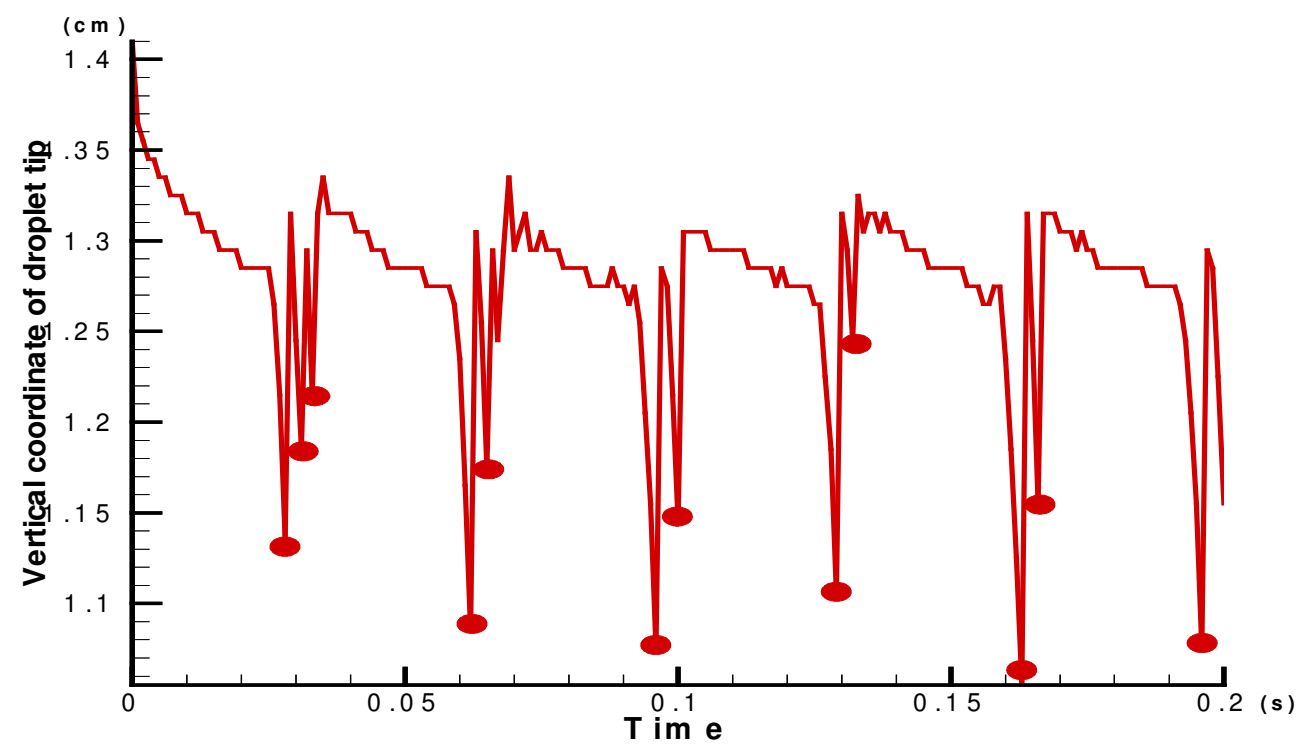

$5.22(\mathrm{a})$ 


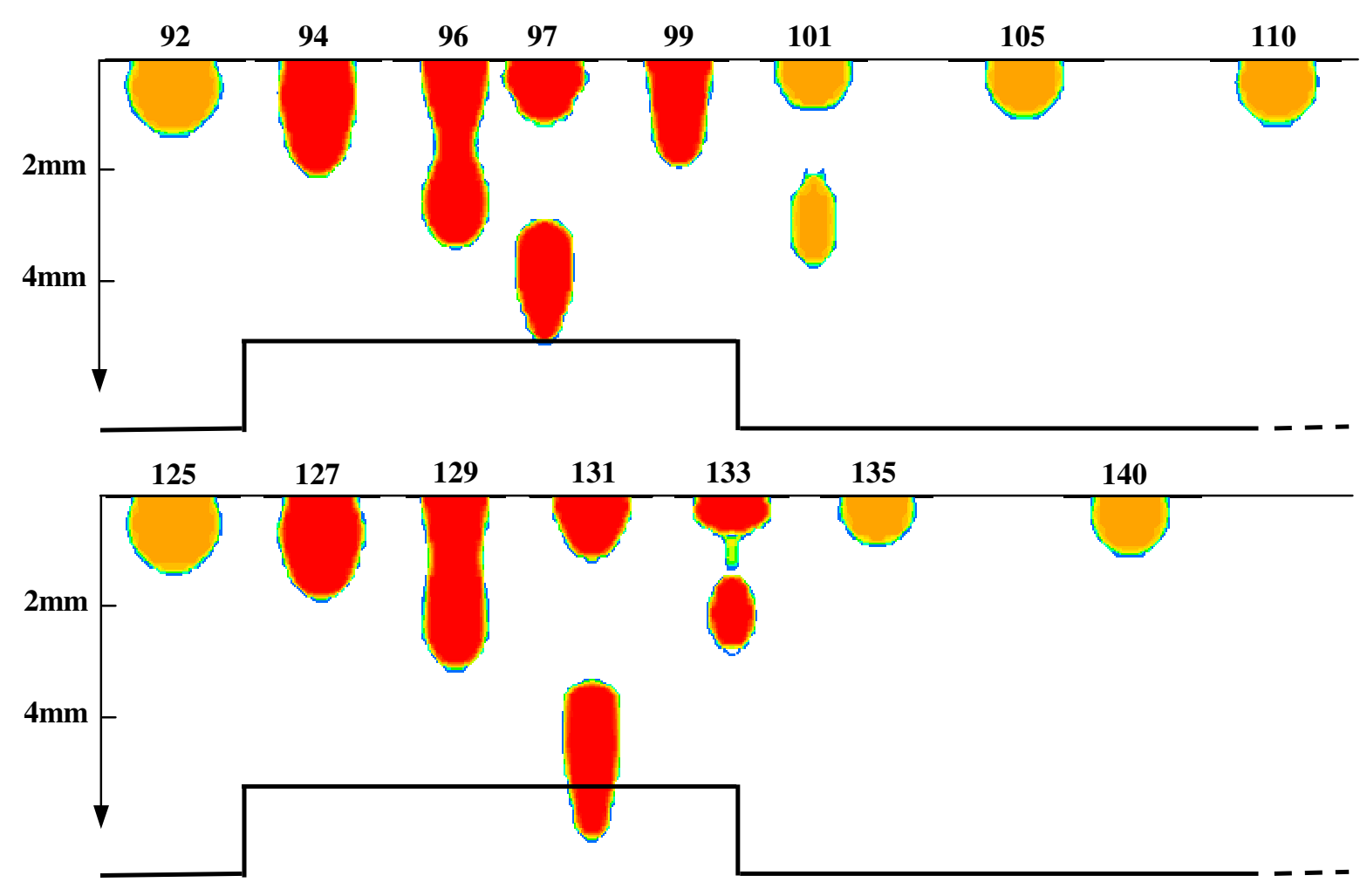

$5.22(b)$

Figure 5.22 Metal transfer with MDPP under conventional pulsed current GMAW:

(a) Vertical coordinate of droplet tip (b) Droplet profiles

Furthermore, calculations are performed for the modified pulsed current GMAW with same amount of the average current $(100 \mathrm{~A})$, pulse cycle frequency $(30 \mathrm{~Hz})$, base current (40A). The single pulse with peak current of 300A, which used in conventional pulsed current GMAW, is divided into two parts - the exciting pulse with peak current of $220 \mathrm{~A}$ on duration of $5 \mathrm{~ms}$ and the detaching pulse with peak current of 300A. The calculated developments for vertical coordinate of droplet tip for different values of time interval $T_{b 2}$ are illustrated in Figure 5.23. 
Metal transfer for a very short time interval $T_{b 2}$ of $2 \mathrm{~ms}$ between the exciting and detaching pulses is shown in Figure 5.23(a). A detaching pulse is added shortly after an exciting pulse. The droplet is bouncing its way up after an exciting pulse when a detaching pulse is enforced. Detaching pulse with value of $300 \mathrm{~A}$ is able to overcome the upward momentum of droplet, detach the droplet, and therefore realize ODPP metal transfer.

The cases for metal transfer under a time interval $T_{b 2}$, which can provide phase match, are shown in Figure 5.23(b) and (c). A detaching pulse is added around the instant when the droplet first bounces to its highest position and starts to move its way down after the exciting pulse. As it has been discussed earlier, phase match takes advantage of synchronization between the downward momentum of droplet and the increase of electromagnetic force to detach the droplet. Phase match is an ideal way to achieve ODPP metal transfer when using modified pulsed current GMAW.

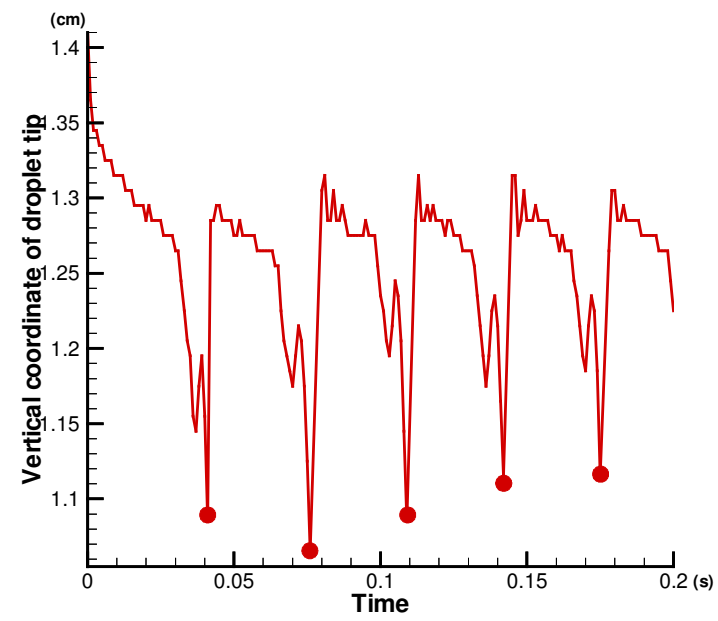

(a)

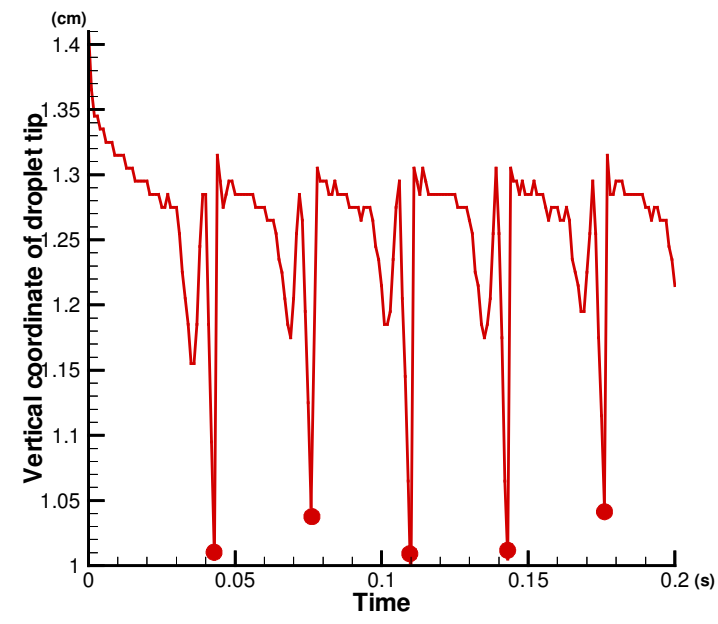

(b) 


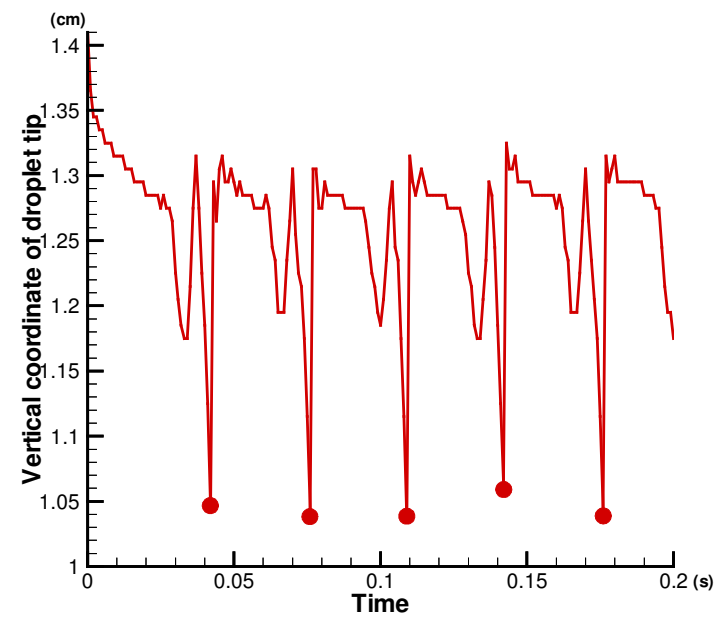

(c)

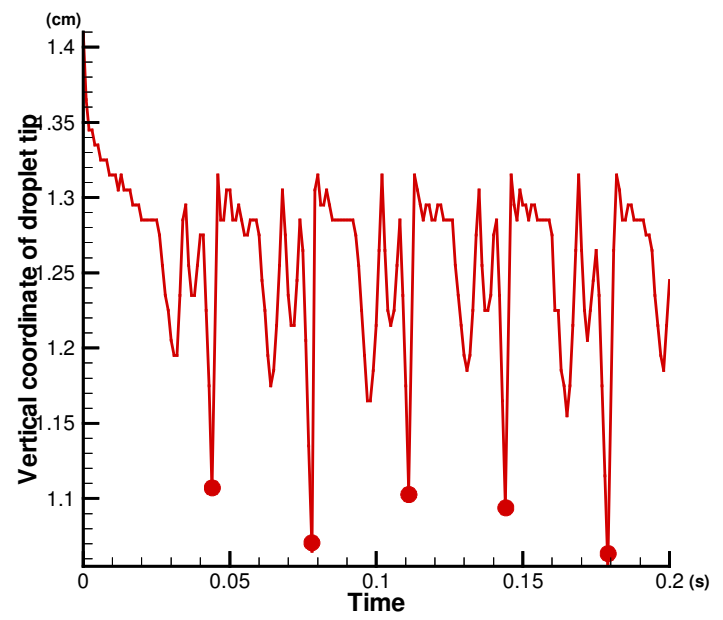

(e)

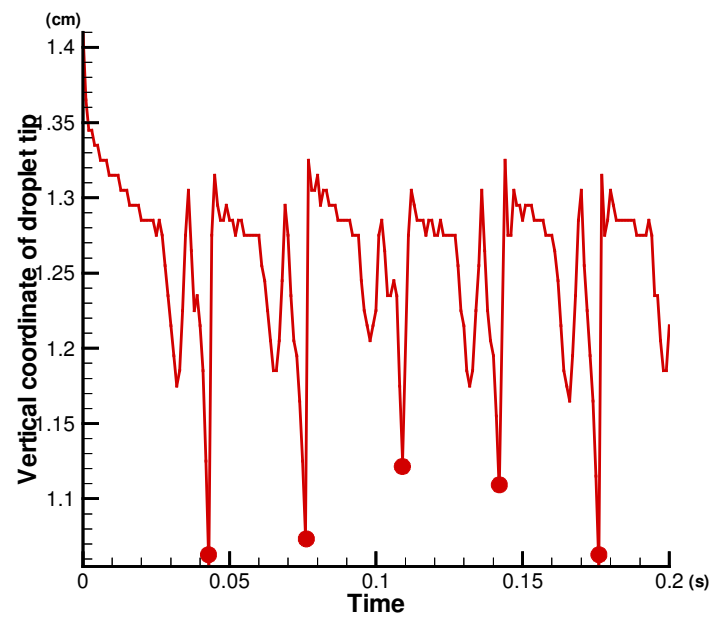

(d)

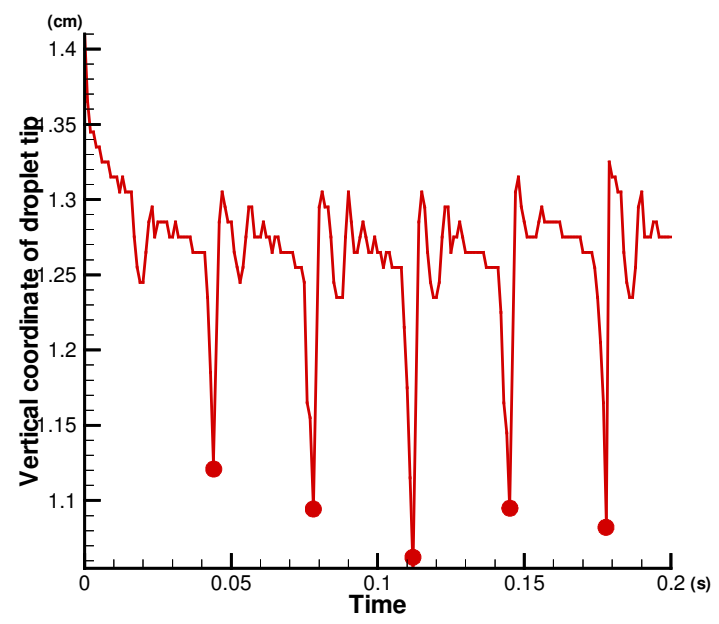

(f)

Figure 5.23 Development for vertical coordinate of droplet tip in modified pulsed current GMAW ( $\left.I_{p 1}=220, I_{p 2}=300, T_{p 1}=5 \mathrm{~ms}\right)$ :
(a) ODPP under $\mathrm{T}_{\mathrm{b} 2}$ of $2 \mathrm{~ms}$
(b) ODPP under $\mathrm{T}_{\mathrm{b} 2}$ of $4 \mathrm{~ms}$
(c) ODPP under $\mathrm{T}_{\mathrm{b} 2}$ of $6 \mathrm{~ms}$
(d) ODPP under $\mathrm{T}_{\mathrm{b} 2}$ of $7 \mathrm{~ms}$
(e) ODPP under $\mathrm{T}_{\mathrm{b} 2}$ of $10 \mathrm{~ms}$
(f) ODPP under $\mathrm{T}_{\mathrm{b} 2}$ of $20 \mathrm{~ms}$ 
As the time interval $T_{b 2}$ between the exciting pulse and the detaching pulse continues to increase, the calculated developments for vertical coordinate of droplet tip are shown in Figure 5.23(d)-(f). Figure 5.23(d) indicates that the droplets just finish the first up and down oscillation cycle after an exciting pulse and start to move upward again before a detaching pulse is added at $T_{b 2}$ of $7 \mathrm{~ms}$. Then, as the time interval $T_{b 2}$ continues rising to $10 \mathrm{~ms}$, a droplet is on its way down the second time after an exciting pulse before a detaching pulse is added and detaches the droplet. As the oscillation cycle continues, the momentum of droplet oscillation is decreased dramatically with the decreasing amplitude of vibration. As a detaching pulse is added $20 \mathrm{~ms}$ later after an exciting pulse (Figure 5.23f), the influence coming from the momentum of droplet oscillation, which is induced by exciting pulse, can almost be neglected. Figure 5.23 shows that ODPP metal transfer mode can be achieved for any value of $T_{b 2}$, i.e., the calculated operating range of $T_{b 2}$ extends to the whole selectable range when using the modified pulsed current GMAW.

The calculated results further show that the modified pulsed current GMAW has advantages over the conventional single pulsed current GMAW. It can avoid accidental droplet detachment, which occurred under the conventional single pulsed current GMAW. Also, the calculated results confirm the above theoretical analysis that the increase of detaching pulse peak current provides strengthened detachment force, assists the droplet detachment, and therefore extends the operating range of $T_{b 2}$ having ODPP metal transfers. 
The wider operating range of $T_{b 2}$ provides more flexibility in achieving the desirable metal transfer mode. However, the use of higher detaching pulse peak current accelerates droplets and produces droplets with higher velocity toward a welding pool. When the average current and droplet detachment frequency are constant, the welding processes produce a detached droplet with same amount of mass. For detached droplets having the same amount of mass, the higher velocity droplets represent higher momentum, stronger impact on a welding pool and a bigger splash. In order to achieve high quality welding, the detached droplets with lower velocity are more favorable.

The velocities of droplets at the tip of electrode are calculated for the above cases that use the different value of detaching pulse current under same preset parameters including average current, pulse frequency, base current, and exciting pulse current. Figure 5.24 shows a comparison of the velocities toward the welding pool as the detaching pulse current increases from $220 \mathrm{~A}$ to $300 \mathrm{~A}$. When the detaching pulse current is $220 \mathrm{~A}$ (model case), the calculated droplet velocity toward the welding pool is between $40 \mathrm{~cm} / \mathrm{s}$ and $55 \mathrm{~cm} / \mathrm{s}$ at the moment of droplet detachment from the electrode. When the detaching pulse current increases to $250 \mathrm{~A}$, the calculated droplet velocity toward the welding pool is between $50 \mathrm{~cm} / \mathrm{s}$ to $70 \mathrm{~cm} / \mathrm{s}$ at the moment of droplet detachment. As the detaching pulse current continues rising to $300 \mathrm{~A}$, the calculated droplet velocity toward the welding pool rises between $70 \mathrm{~cm} / \mathrm{s}$ to $90 \mathrm{~cm} / \mathrm{s}$ at the moment of droplet detachment from the electrode. The calculated results support the above theoretical analysis that the 
use of higher detaching pulse peak current accelerates droplets and produces droplets with higher velocity toward the welding pool.
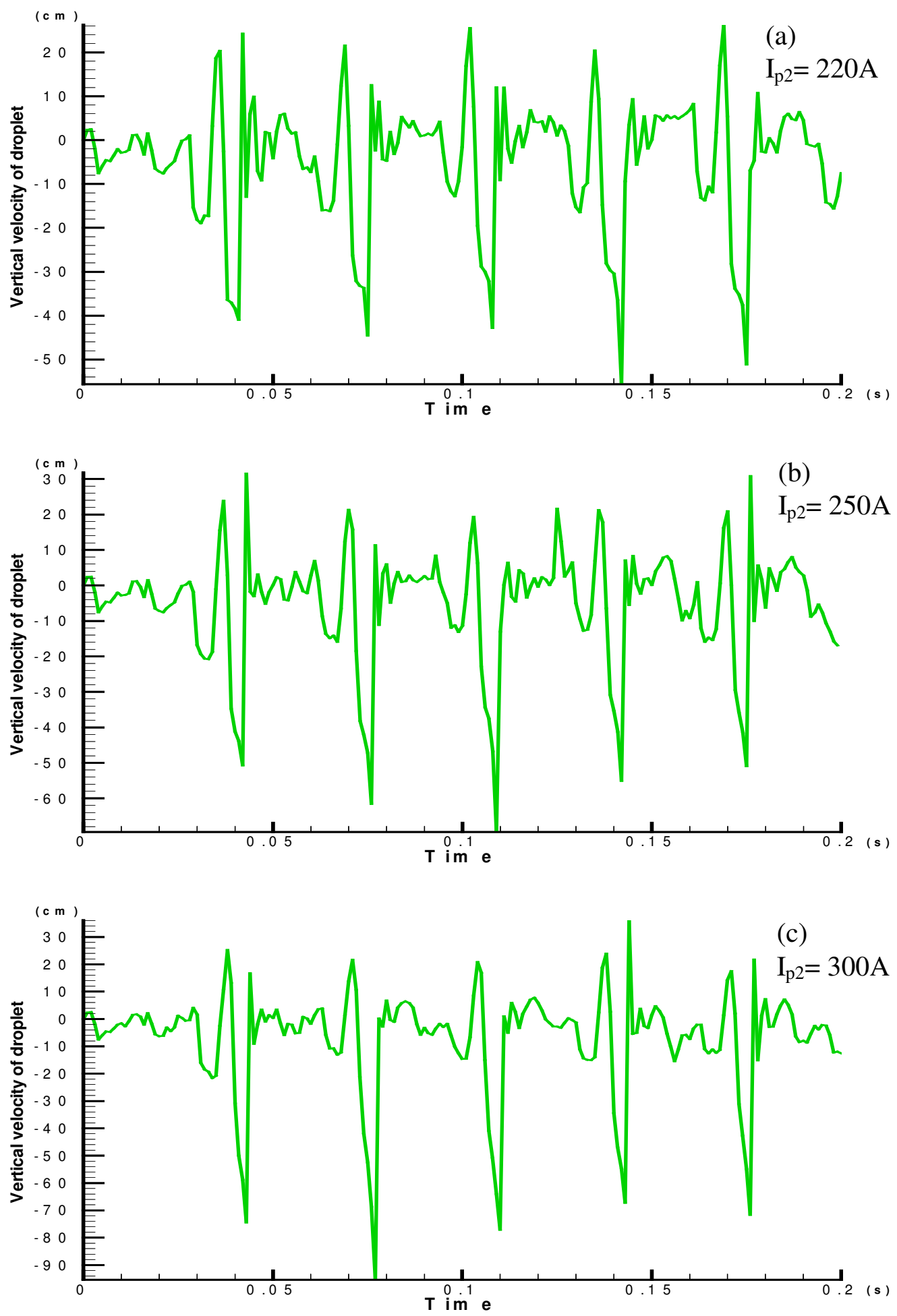

Figure 5.24 Drop velocities toward welding pool under different detaching pulse current 
Hence, choosing a higher detaching pulse current increases flexibility in achieving ODPP metal transfer mode if a welding process focuses on a periodic, controllable metal transfer process and has fewer requirements on heat sensitivity and spattering. The numerical analysis show that the modified pulsed current GMAW has much more flexibility to obtain desirable ODPP metal transfer process by adjustment of parameters than single-pulsed conventional GMAW.

\subsubsection{Comparison with Experiment}

Figure 5.25 shows the comparison between the calculated results and experimental [64] data provided by Zhang, E and Kovacevic when the proposed active metal transfer control is employed. The average current is set at $100 \mathrm{~A}$ and $165 \mathrm{~A}$,
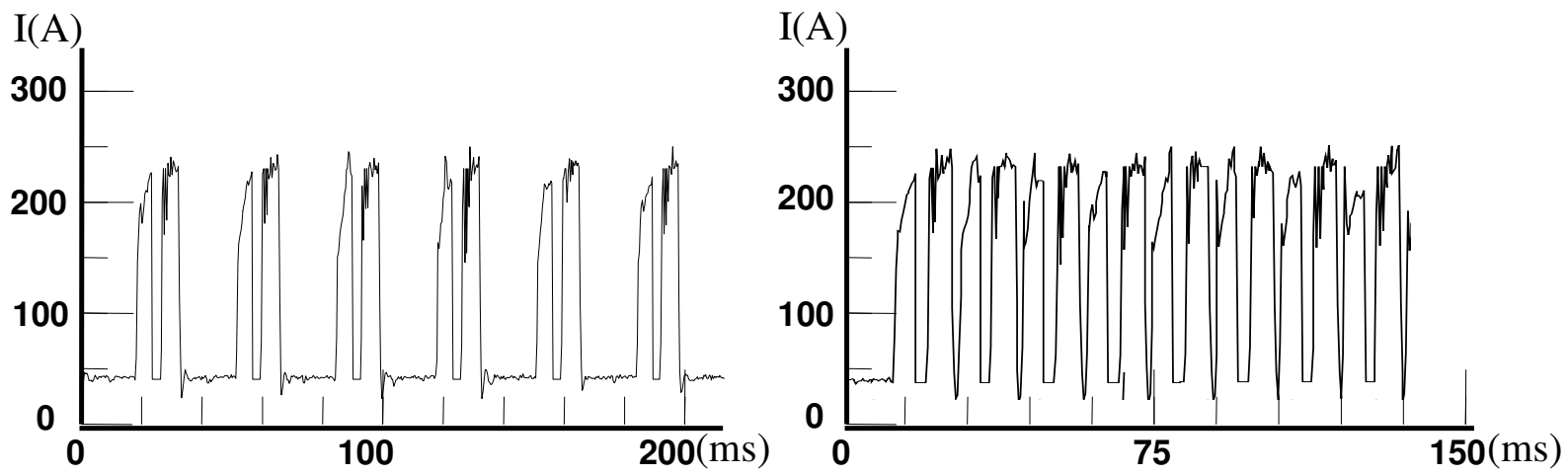

$5.25(a)$ 

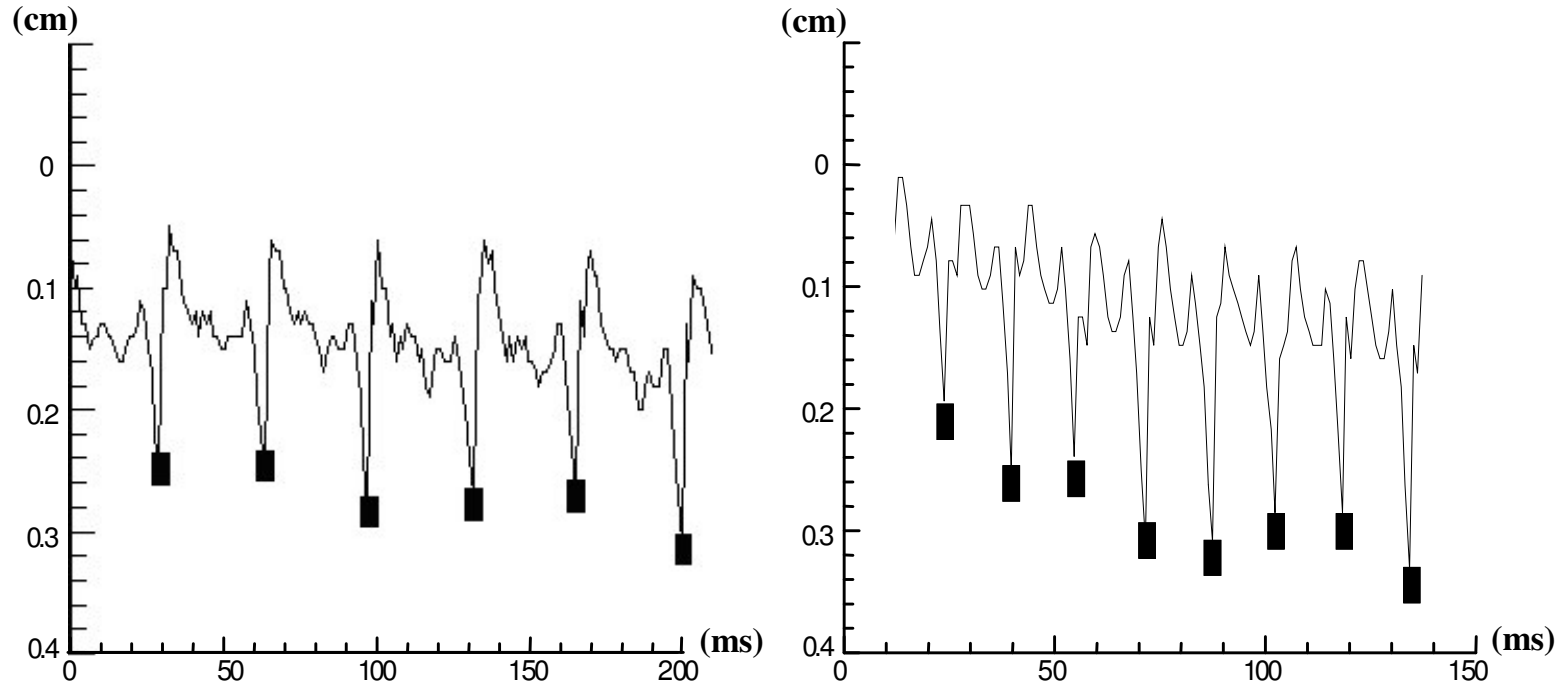

\section{$5.25(b)$}
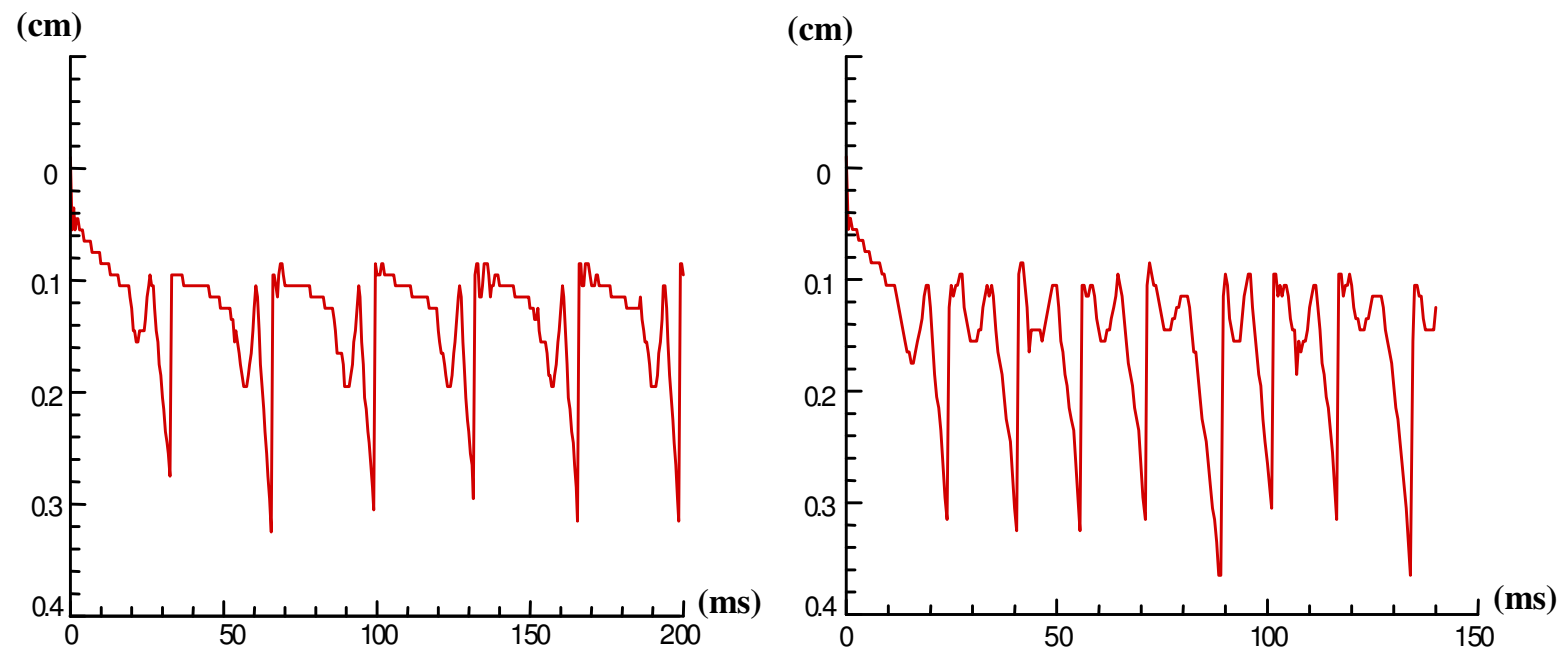

$$
5.25(\mathrm{c})
$$

Figure 5.25 Comparison between the calculated results and experimental data:

(a) Current waveforms (b) Vertical coordinate of droplet tip from experiment (c) Vertical coordinate of droplet tip from calculation 
respectively. The transfer frequency is $30 \mathrm{~Hz}$ for $100 \mathrm{~A}$ and $65 \mathrm{~Hz}$ for $165 \mathrm{~A}$. According to experimental data taken from the work of Zhang, E, and Walcott [63], the wire feed speed is given as $110 \mathrm{in} / \mathrm{min}(46.5 \mathrm{~mm} / \mathrm{s})$ for $100 \mathrm{~A}$ and $180 \mathrm{in} / \mathrm{min}(76.2 \mathrm{~mm} / \mathrm{s})$ for $165 \mathrm{~A}$. The waveform of the welding current signal is shown in Figure 5.25(a). The measured and calculated vertical coordinates of the droplet tip, which increase with time, are shown in Figure 5.25(b) and (c) respectively. The calculated results agree well with the experimental data. One drop per pulse cycle is realized under these two sets of data provided by the experiment.

\subsection{Summary}

A modified pulsed current GMAW, which has been proposed to improve the quality of gas metal arc welding, is presented and simulated in this chapter. This new approach takes advantage of the downward momentum of droplet oscillation to reduce the current level, which is required to detach the droplet and to realize ODPP metal transfer. The calculations demonstrated the effectiveness of the proposed approach and its advantage over the conventional pulsed current GMAW. The phase match, which has been a determining factor to the success of this new technique, can be diagnosed and calculated by our numerical program.

Some important qualitative conclusions can be drawn according to present calculations: 
(1) There exist operating ranges of the pulse signal parameters including the time interval $T_{b 2}$, exciting pulse duration $T_{p 1}$, peak current $I_{p}$ and pulse frequency $f$.

(2) The operating range of $T_{b 2}$ is shifted slightly to a smaller value without much change of time interval as the exciting pulse duration $T_{p 1}$ is decreased within a certain range.

(3) The operating range of the time interval $T_{b 2}$ is decreased as the pulse frequency $f$ is increased or the peak current $I_{p}$ is decreased.

(4) The increase of detaching pulse peak current, with the other parameters including the average current, pulse frequency, base current unchanged, extends the operating range of $T_{b 2}$.

(5) The use of higher detaching pulse peak current accelerates droplet and produces a droplet with higher velocity toward the welding pool.

Copyright @ Ge Wang 2007 


\section{Chapter 6}

\section{Conclusions}

In this dissertation, the dynamic process of metal transfer in GMAW has been studied numerically. A numerical program employing advanced techniques in CFD, which include a two-step projection method, volume of fluid method and continuum surface force model, has been developed by incorporating the electromagnetic field calculation. In the present study, the effects of surface tension, electromagnetic and gravitational forces are considered as the major forces to influence the process of the molten droplet development and detachment. The assumption of Gaussian current density distribution on the droplet surface is proposed. The calculated results show very good agreement with the experimental data under this assumption.

The numerical analysis for metal transfer process in constant current GMAW is performed first. At low currents, the gravitational force dominates the droplet detachment process and produces large droplets. Globular metal transfer with big spattering occurs. At high currents, the electromagnetic force dominates the droplet detachment process and produces small droplets. Spray metal transfer with significant heat and high velocity droplets occur. There is a sharp transition current range between globular metal transfer and spray metal transfer. Due to the competition between the gravitational force and 
electromagnetic force, a bifurcation in the droplet detachment frequency and the droplet size occurs over this transition current range.

The calculation carried out in this work is the first to catch the transition zone between globular and spray metal transfer accurately and to demonstrate the unique metal transfer characteristic over this transition range by numerical simulation. Also, the numerical analysis based on the calculation provides a better understanding of and theoretical support for the physical mechanisms that influence the metal transfer modes. The calculated results further confirm that the natural detachment of droplet (by gravitational force and electromagnetic force) with size similar to the electrode diameter only happens under the spray metal transfer at high welding currents.

In order to improve welding quality, a novel pulsed current GMAW that has been proposed according to experiment is simulated and analyzed by our numerical program. A pulse cycle is composed of two pulses: exciting pulse and detaching pulse in this new technique. The proposed method takes advantage of synchronization between the downward momentum of the oscillating drop and the increased electromagnetic force to realize ODPP metal transfer for currents lower than transition current in GMAW. The calculation not only shows the effectiveness of the proposed approach to achieve single droplet detached by every pulse cycle, but also provides an effective means to diagnose the optimum operation parameters. The time interval $T_{b 2}$ is identified as a crucial parameter to realize phase match and therefore to achieve ideal ODPP metal transfer when using this modified pulsed current GMAW. 
The calculations show that there exist operating ranges of the pulse signal parameters including the time interval $T_{b 2}$, exciting pulse duration $T_{p 1}$, peak current $I_{p}$ and pulse frequency $f$. The operating range of $T_{b 2}$ is shifted slightly to a smaller value without much change of time interval as the exciting pulse duration $T_{p 1}$ is decreased within a certain range. Also, the calculated results indicate the operating range of the time interval $T_{b 2}$ is decreased as the pulse frequency $f$ is increased or the peak current $I_{p}$ is decreased. The calculations further demonstrate that the increase of detaching pulse peak current, with the other parameters including the average current, pulse frequency, base current unchanged, extends the operating range of $T_{b 2}$ to have ODPP metal transfers. At the same time, the calculated results prove the use of higher detaching pulse peak current accelerates droplets and produces droplets with higher velocity toward a welding pool.

Comparison with the conventional single pulsed GMAW, calculations show that the modified pulsed current GMAW not only can achieve ODPP metal transfer for peak current lower than transition current, but also has much more flexibility to obtain desirable metal transfer process by adjustment of parameters. The robustness of the metal transfer process in GMAW provided by this new technology is significantly improved in comparison with conventional single pulsed GMAW process.

Also, the existence of the operating ranges for the pulse signal parameters shows the flexibility and stability of this modified pulsed current GMAW to produce ODPP metal transfer. The numerical analysis and parameter diagnoses provided by this 
dissertation not only give this new gas metal arc welding technique theoretical support, but also are very important to make it feasible in industry.

Copyright @ Ge Wang 2007 


\section{References}

1. J. H. Waszink and L. H. J. Graat, "Experimental Investigation of the Forces Acting on a Drop of Weld Metal", Welding Journal, 1983, Vol.62, pp.108s-116s

2. J. H. Waszink and M. J. Piena, "Experimental Investigation of Drop Detachment and Drop Velocity in GMAW”, Welding Journal, 1986, Vol.65, pp.289s-298s

3. W. G. Essers and R. Walter, "Heat Transfer and Penetration Mechanisms with GMA and Plasma-GMA Welding”, Welding Journal, 1981, Vol.60, pp.37-42

4. B. Ogunbiyi and J. Norrish, "GMAW Metal Transfer and Arc Stability Assessment", 8th Conf. Computer Technology in Welding TWI, 1996, pp.9-12

5. L. P. Jin, J. C. Lin and Z. H. Wang, "The Effect of the Dynamic Behavior of Welding Rectifiers on Spatter", Welding International, Vol.1, pp.129-133

6. J. F. Lancaster, "Metal Transfer and Mass Flow in the Weld Pool", The Physics of Welding, 2nd ed., 1986, Pergamon Press, Oxford.

7. Welding Handbook, 8th edition, Vol.2, Welding Processes, AWS, 1991

8. A. Lesnewich, "Control of Melting Rate and Metal Transfer in Gas-Shielded Metal Arc Welding”, Welding Journal, 1958, Vol.37, pp.418s-425s

9. S. W. Simpson, P. Zhu, and M. Rados, "Metal Transfer Modes in Gas Metal Arc Welding", Proc. 42nd Nat. Weld. Conf., 1994, Vol.31, pp.1-8

10. L.A. Jones, T.W. Eagar, and J.H. Lang, "Images of a Steel Electrode in Ar- $2 \% \mathrm{O}_{2}$ Shielding during Constant Current Gas Metal Arc Welding”, Welding J., 1998, Vol.77, pp.135s-141s 
11. S. K. Choi, C. D. Yoo and Y. S. Kim, "Dynamic Simulation of Metal Transfer in GMAW, Part 2: Short-Circuit Transfer Mode”, Welding J., 1998, Vol.77, pp.45s-51s

12. S. T. Eickhoff and T. W. Eagar, "Characterization of Spatter in Low-Current GMAW of Titanium Alloy Plate”, Welding Journal, 1990, Vol.69, pp.382s-388s

13. L. A. Jones, T. W. Eagar and J. H. Lang, "Investigation of Drop Detachment Control in Gas Metal Arc Welding", The 3rd International Conference on Trends in Welding Research, TN, 1992, pp.1009-1013

14. J. C. Needham, "Control of Transfer in Aluminum Consumable Electrode Welding", Physics of Welding Arc, 1962, pp.114-124

15. C. D. Allemand, "A Method of Filming Metal Transfer in Welding Arcs", Welding Journal, January 1985, Vol.64, pp.45-47

16. J. A. Lawrence et al, "Dynamic Electrode Forces in Gas Metal Arc Welding", MIT Thesis, October 1995

17. S. Rhee and E. Kannatey, "Observation of Metal Transfer during Gas Metal Arc Welding”, Welding Journal, 1992, Vol.71, pp.381s-386s

18. R. Kovacevic, M. Erlenbeck, H. Beardsley and Y.M. Zhang, "Real Time Image Acquisition and Processing of Metal Transfer", 77th Annual American Welding Society Convention, April 1996

19. G. Adam and T.A. Siewert, "Sensing of GMAW Droplet Transfer Modes Using ER100s-1 Electrode”, Welding J., 1990, Vol.69, pp.103s-108s

20. J. A. Johnson, N. M. Carlson, H. B. Smartt and D. E. Clark, "Process Control of GMAW: Sensing of Metal Transfer Mode", Welding Journal, 1991, Vol.70, pp.91s99s 
21. H. B. Smartt et al, "Gas Metal Arc Welding Process Sensing and Control, Advances in Welding Science and Technology", Proc. Int. Conf. On Trends in Welding Research, Tennessee, pp.461-65, 1986

22. X. Li, S. W. Simpson and M. Rados, Sci. Technol. Weld. Joining, 2000, Vol.5, pp.7179

23. F. Manz, "Welding Arc Sounds", Weld. Journal, 1981, Vol.60, pp.23s-27s

24. Q. Lin, X. Li and S. W. Simpson, "Metal Transfer Measurements in Gas Metal Arc Welding”, J. Phys. D: Appl. Phys., 2001, Vol.34, pp.347-353

25. W. J. Greene, “An Analysis of Transfer in Gas-Shielded Welding Arcs”, Trans. AIEE Part II, 1960, Vol.79, pp.194-203

26. J. C. Amson, “An Analysis of the Gas Shielded Consumable Metal Arc Welding System”, British Welding Journal, Vol.41, pp.232-249

27. J. C. Amson, "Experimental Investigation of the Forces Acting on a Drop of Weld Metal”, Br. J. Appl. Phys., 1965, Vol.16, pp.1169-1179

28. C. J. Allum, "Metal Transfer in Arc Welding as a Varicose Instability: I. Varicose Instability in a Current-Carrying Liquid Cylinder with Surface Change", J. Phys. D: Appl. Phys., 1985, Vol.18, pp.1431-1446

29. C. J. Allum, "Metal Transfer in Arc Welding as a Varicose Instability: II. Development of the Model of Arc Welding", J. Phys. D, 1985, Vol.18, pp.1447-1468

30. S. Rhee, and A. E. Kannatey, "Analysis of Arc Pressure Effect on Metal Transfer in Gas Metal Arc Welding”, J. Phys. D: Appl. Phys., 1991, Vol.24, pp.5068-5075

31. J. H. Choi, J. Lee and C. D. Yoo, "Dynamic Force Balance Model for Metal Transfer Analysis in Arc Welding”, J. Phys. D: Appl. Phys., 2001, Vol.34, pp.2658-2664 
32. V. A. Nemchinsky, "Size and Shape of the Liquid Droplet at the Molten Tip of an Arc Electrode”, J. Phys. D: Appl. Phys., 1994, Vol.27, pp.1433-1442

33. T. M. Joo, C. D. Yoo and T. S. Lee, "Effects of Welding Conditions on Molten Drop Geometry in Arc Welding", J. of Eng. for Industry, ASME, 1996, Vol.118, pp.623627

34. S. W. Simpson and P. Zhu, "Formation of Molten Droplets at a Consumable Anode in an Electric Welding Arc”, J. Phys. D, 1995, Vol.28, pp.1594-1600

35. L. A. Jones, T. W. Eagar and J. H. Lang, "Magnetic Forces Acting on Molten Drops in Gas Metal Arc Welding”, Journal of Physics D: Applied Physics, Jan 1998, Vol.31, pp.93-106

36. L. A. Jones, T. W. Eagar and J.H. Lang, "A Dynamic Model of Drops Detaching From a Gas Metal Arc Welding Electrode”, Journal of Physics D: Applied Physics, Jan 1998,Vol.31, pp.107-123

37. H. Maruo, Y. Hirata and N. Goto, "Bridging Transfer Phenomena of Conductive Pendent Drop: The Effect of Electro-magnetic pinch force on the bridging transfer", Quarterly Journal of JWS, 1992, Vol.10, pp.243-250

38. J. Haidar and J. J. Lowke, "Predictions of Metal Droplet Formation in Arc Welding", J. Phys. D: Appl. Phys., 1996, Vol.29, pp.2951-2960

39. J. Haidar and J. J. Lowke, "Effect of $\mathrm{CO}_{2}$ Shielding Gas on Metal Droplet Formation in Arc Welding", IEEE Transactions on Plasma Science, Oct 1997, Vol.25, pp.931936

40. J. Haidar, "An Analysis of the Formation of Metal Droplets in Arc Welding”, J. Phys. D, 1998, Vol.31, pp.1233-1244 
41. H. G. Fan and R. Kovacevic, "Dynamic Analysis of Globular Metal Transfer in Gas Metal Arc Welding-A Comparison of Numerical and Experimental Results”, J. Phys. D: Appl. Phys., 1998, Vol.31, pp.2929-2941

42. H. G. Fan, and R. Kovacevic, "Droplet Formation, Detachment, and Impingement on the Molten Pool in Gas Metal Arc Welding”, Metall. Mater. Trans. B, 1999, Vol. 30B, pp.791-801

43. S. K. Choi, C. D. Yoo and Y. S. Kim, "Dynamic Simulation of Metal Transfer in GMAW, Part 1: Globular and Spray Transfer Modes”, Welding Journal, 1998, Vol.77, pp.38s-44s

44. S. K. Choi, Y. S. Kim and C. D. Yoo, "Dimensional Analysis of Metal Transfer in GMA Welding”, J. Phys. D, 1999, Vol.32, pp.326-34

45. Y. Wang and H. L. Tsai, "Impingement of Filler Droplets and Weld Pool Dynamic during Gas Metal Arc Welding Process”, Int. J. Heat and Mass Transfer, 2001, Vol.44, pp.2067-2080

46. Y. Wang and H. L. Tsai, "Effects of Surface Active Elements on Weld Pool Fluid Flow and Weld Penetration in GMAW”, Metall. Mater. Trans. B, 2001, Vol.32, pp. $501-515$

47. Y. Wang, H. L. Tsai, S. P. Marin and P. C. Wang, Proceeding of IMECE'02, 2002 ASME International Mechanical Engineering Congress \& Exposition, New Orleans, Louisiana, Nov. 2002, pp.17-22

48. S. Ueguri, K. Hara and H. Komura, "Study of Metal Transfer in Pulsed GMA Welding”, Welding Journal, 1985, Vol.64, pp.242s-250s 
49. M. Amin, "Pulsed Current Parameters for Arc Stability and Controlled Metal Transfer in Arc Welding”, Metal Construction, 1983, Vol.15, pp.272-278

50. L. Quintino and C. J. Allum, "Pulsed GMAW: Interactions Between Process Parameters-Part 1”, Welding Met. Fabrication, 1984, Vol.3, pp.85-89

51. L. Quintino and C. J. Allum, "Pulsed GMAW: Interactions Between Process Parameters -Part 2”, Welding Met. Fabrication, 1984, Vol.4, pp.126-129

52. Z. Smati, "Automatic Pulsed GMA Welding”, Met. Construction, 1986, Vol.18, pp. $38-44$

53. N. Jacobsen, "Monopulse Investigation of Drop Detachment in Pulsed Gas Metal Arc Welding”, J. Phys. D, 1992, Vol.25, pp.783-797

54. Y. S. Kim, "Metal Transfer in Gas Metal Arc Welding", Ph.D Thesis, 1989, MIT, Cambridge, MA

55. Y. S. Kim and T. W. Eagar, "Metal Transfer in Pulsed Current Gas Metal Arc Welding”, Welding J., 1993, Vol.72, pp.279s-287s

56. S. K. Choi, C. D. Yoo and Y. S. Kim, “The Dynamic Analysis of Metal Transfer in Pulsed Current Gas Metal Arc Welding”, J. Phys. D: Appl. Phys., 1998, vol.31, pp. $207-215$

57. V. A. Nemchinsky, "Electrode Melting During Arc Welding with Pulsed Current”, J. Phys. D: Appl. Phys., 1998, Vol.31, pp. 2797-2802

58. G. Wang, P. G. Huang and Y. M. Zhang, "Numerical Analysis of Metal Transfer in Gas Metal Arc Welding”, Metall. Mater. Trans. B, 2003, Vol.34B, pp.345-353 
59. G. Wang, G. Huang, and Y. M. Zhang, "Numerical Analysis of Metal Transfer in Gas Metal Arc Welding Under Modified Pulsed Current Conditions," Metallurgical and Materials Transactions B, 2004, Vol.35, pp.991-999

60. D. B. Kothe, R. C. Mjolsness, and M. D. Torrey, "RIPPLE: A Computer Program for Incompressible Flows with Free Surfaces”, Los Alamos National Laboratory, 1994, LA-12007-MS.

61. C. W. Hirt, and B. D. Nichols, "Volume of Fluid (VOF) Method for the Dynamics of Free Boundaries”, J. Comp. Phys., 1981, Vol.39, pp.201-225

62. J. U. Brackbill, D. B. Kothe, and C. Zemach, “A Continuum Method for Modeling Surface Tension”, J. Comp. Phys., 1992, Vol.100, pp.335-54

63. Y. M. Zhang, Liguo E and B. L. Walcott, "Robust Control of Pulsed Gas Metal Arc Welding”, ASME Journal of Dynamic Systems, Measurement, and Control, 2002, Vol.124, pp. 281-289

64. Y. M. Zhang, Liguo E and R. Kovacevic, "Active Metal Transfer Control by Monitoring Excited Droplet Oscillation”, Welding J., 1998, Vol. 77, pp. 388s-395s

65. Y. M. Zhang, and P. J. Li, "Modified Active Control of Metal Transfer and Pulsed GMAW of Titanium", Welding Journal, 2001, Vol.80, pp.54s-61s

66. F. H. Harlow and J. E. Welch, "Numerical Calculation of Time Dependent Viscous Incompressible Flow of Fluid with Free Surface", Physics Fluids, 1965, Vol.8, pp.2182-2189

67. J. Glimm, D. Marchesin, and O. Mcbryan, "A Numerical Method for Two Phase Flow with an Unstable Interface”, J. Comp. Phys., 1981, Vol.39, pp.179-200 
68. Y. S. Kim and T. W. Eagar, "Analysis of Metal Transfer in Gas Metal Arc Welding", Welding Journal, 1993, Vol.72, pp.269-278

69. J. Ma and R. L. Apps, “Analyzing Metal Transfer during MIG Welding”, Welding and Metal Fabrication, 1983, Vol.51, pp.119-128

70. H. R. Castner, "Gas Metal Arc Welding Fume Generation Using Pulsed Current", Welding Journal, 1995, Vol.74, pp.59-68s 


\section{Vita}

The author was born on November 10, 1971 in Dongying, ShanDong Province, P. R. China. She enrolled in Xi' an JiaoTong University, Xi' an, P. R. China, in September 1989 and received her Bachelor's degree in Engineering from Xi'an University in July 1993. After graduation, she worked at the Department of Chemical Engineering, Northwest University, as a lecturer for two years. In 1995, she continued her graduate studies at the department of Aerodynamics at Chinese Academy of Science, Beijing, China and earned her Master degree in June 1998. Then, she came to the United States to pursue her Ph.D. degree and enrolled at the Department of Mechanical Engineering, University of Kentucky. The author has 9 professional publications in journals or technical conferences.

Ge Wang

7/10/2007 